Role of nucleation and growth in two-phase microstructure formation

by

\title{
Jong Ho Shin
}

A dissertation submitted to the graduate faculty

in partial fulfillment of the requirements for the degree of

DOCTOR OF PHILOSOPHY

\author{
Major: Materials Science and Engineering \\ Program of Study Committee: \\ - Rohit K. Trivedi, Major Professor \\ Brian Gleeson \\ L. Scott Chumbley \\ Palaniappa A. Molian \\ Dennis R. Vigil
}

Iowa State University

Ames, Iowa

2007

Copyright $(\mathcal{O}$ Jong Ho Shin, 2007. All rights reserved. 


\section{TABLE OF CONTENTS}

ABSTRACT iv

CHAPTER 1: INTRODUCTION 1

1 General introduction 1

2 Dissertation organization 4

References 5

CHAPTER 2: BACKGROUND

1 Nucleation $\quad 7$

1.1 Driving force for solidification 8

1.2 Homogeneous nucleation 9

$\begin{array}{ll}1.3 \text { Heterogeneous nucleation } & 10\end{array}$

2 Interface stability $\quad 21$

3 Cellular growth 25

4 Convection effects $\quad 27$

5 Band structure in peritectic alloys $\quad 28$

6 Eutectic structure $\quad 29$

References $\quad 35$

CHAPTER 3: MODELLING OF MICROSTRUCTURE EVOLUTION IN PERITECTIC SYSTEMS $\quad 37$

$\begin{array}{ll}\text { Abstract } & 37 \\ 1 \text { Introduction } & 37\end{array}$

1 Introduction $\quad 38$

2 Experimental procedure $\quad 39$

3 Theoretical modelling and experimental results $\quad 40$

$\begin{array}{ll}3.1 \text { Composite microstructure } & 43\end{array}$

3.2 Discrete band formation $\quad 45$

3.3 Nucleation characteristics $\quad 51$

3.4 Partial bands due to growth competition $\quad 53$

3.5 Complex microstructures $\quad 55$

4 Conclusions $\quad 58$

Acknowledgments $\quad 58$

References $\quad 62$

CHAPTER 4: HETEROGENEOUS NUCLEATION IN PERITECTIC
SYSTEMS

$\begin{array}{ll}\text { Abstract } & 63 \\ 1 \text { Introduction } & 63\end{array}$

1 Introduction $\quad 64$

2 Experimental procedure $\quad 66$

$\begin{array}{ll}3 \text { Results } & 72 \\ & 72\end{array}$

$\begin{array}{ll}3.1 \text { Nucleation undercooling } & 72\end{array}$

$\begin{array}{ll}3.2 \text { Contact angles } & 73\end{array}$ 
4 Discussion $\quad 81$

$\begin{array}{ll}\text { 4.1 Nucleation at the WSL junction } & 81\end{array}$

4.2 Nucleation undercooling $\quad 85$

4.3 Nucleation mechanism $\quad 90$

4.4 Nucleation and microstructure evolution 92

5 Conclusions $\quad 98$

Acknowledgments $\quad 99$

References $\quad 99$

$\begin{array}{ll}\text { Appendix } & 100\end{array}$

\section{CHAPTER 5: COUPLED AND SIMULTANEOUS GROWTH
IN PERITECTIC ALLOYS}

$\begin{array}{lr}\text { Abstract } & 102\end{array}$

1 Introduction $\quad 103$

2 Convection effects $\quad 106$

3 Experimental procedure $\quad 110$

4 Experimental results $\quad 110$

4.1 The stability of composite microstructures $\quad 111$

4.2 The effect of tube materials on microstructure 116

$\begin{array}{ll}5 \text { Discussion } & 117\end{array}$

5.1 Coupled growth in peritectic alloys $\quad 117$

5.2 Coupled growth of Sn-Cd alloy in the tantalum tube 121

6 Conclusions $\quad 127$

$\begin{array}{lr}\text { Acknowledgements } & 128\end{array}$

$\begin{array}{ll}\text { References } & 128\end{array}$

CHAPTER 6: GENERAL CONCLUSIONS

\section{APPENDIX: DETERMINING SOLUTE DIFFUSION COEFFICIENT
BY DIRECTIONAL SOLIDIFICATION TECHNIQUE}

$\begin{array}{ll}\text { Abstract } & 133\end{array}$

1 Introduction 134

2 Experimental procedure $\quad 135$

3 Experimental results $\quad 136$

3.1 Morphology of $\mathrm{Pb}-2.0 \mathrm{wt} \% \mathrm{Sb} \quad 136$

3.2 Solute distribution 138

$\begin{array}{ll}4 \text { Discussion } & 142\end{array}$

4.1 Calculation of diffusion coefficient 142

$\begin{array}{ll}4.2 \text { Comparison with literature data } & 146\end{array}$

4.3 Comparison with determination of $\mathrm{D}_{l}$ in Al-Cu alloys $\quad 147$

$\begin{array}{ll}5 \text { Conclusions } & 148\end{array}$

$\begin{array}{lr}\text { Acknowledgments } & 150\end{array}$

$\begin{array}{ll}\text { References } & 150\end{array}$ 


\begin{abstract}
During the directional solidification of peritectic alloys, a rich variety of two-phase microstructures develop, and the selection process of a specific microstructure is complicated due to the following two considerations. (1) In contrast to many single phase and eutectic microstructures that grow under steady state conditions, two-phase microstructures in a peritectic system often evolve under non-steady-state conditions that can lead to oscillatory microstructures, and (2) the microstructure is often governed by both the nucleation and the competitive growth of the two phases in which repeated nucleation can occur due to the change in the local conditions during growth. In this research, experimental studies in the Sn$\mathrm{Cd}$ system were designed to isolate the effects of nucleation and competitive growth on the dynamics of complex microstructure formation. Experiments were carried out in capillary samples to obtain diffusive growth conditions so that the results can be analyzed quantitatively. At high thermal gradient and low velocity, oscillatory microstructures were observed in which repeated nucleation of the two phases was observed at the wall-solidliquid junction. Quantitative measurements of nucleation undercooling were obtained for both the primary and the peritectic phase nucleation, and three different ampoule materials were used to examine the effect of different contact angles at the wall on nucleation undercooling. Nucleation undercooling for each phase was found to be very small, and the experimental undercooling values were orders of magnitude smaller than that predicted by the classical theory of nucleation. A new nucleation mechanism is proposed in which the clusters of atoms at the wall ahead of the interface can become a critical nucleus when the cluster encounters the triple junction. Once the nucleation of a new phase occurs, the
\end{abstract}


microstructure is found to be controlled by the relative growth of the two phases that give rise to different oscillatory microstructures that depend on the imposed velocity and the size of the sample.

At low thermal gradient to velocity ratio, a steady-state composite microstructure is observed. Two mechanisms of composite microstructure formation were examined: (i) the formation of the peritectic phase in the intercellular region of the primary phase where the solute rejected by the primary phase is absorbed by the peritectic phase. The peritectic phase forms a small distance behind the growing primary phase front. (ii) The second mechanism is the coupled growth of the two phases with a macroscopically planar interface, as in the case of eutectic growth. Detailed studies showed that this composite microstructure, although it appears as a eutectic microstructure, did not grow in the coupled manner at the advancing interface in the Sn-Cd system. However, a new observation was made when experiments were carried out in thin ampoule of Ta. The peritectic phase nucleated at the wall-interface triple junction and grew along the wall, while the primary phase continued to grow at the center, giving rise to a steady-state couple growth at some specific velocity. The mechanism of coupled growth in this case was shown to be operative due to the presence of a finite contact angle at the wall, and this was demonstrated by including the contact angle effect at the wall in the rod eutectic growth model.

The experimental results were summarized to map out the conditions of thermal gradient and velocity on the regimes of composite and oscillatory microstructure formation. The formation of complex time-dependent microstructures was then discussed in terms of the time-dependent dynamics of planar interface growth. 


\section{CHAPTER 1: INTRODUCTION}

\section{General introduction}

Solidification of alloys in peritectic systems represents an important class of twophase pattern formation. A typical phase diagram of a peritectic system is shown in Fig. 1. When an alloy in the two-phase region is solidified, a variety of two-phase microstructures form, which determine the properties of the material. There are several key aspects that require the study of microstructure formation in peritectic systems. (1) Many important alloy systems that show peritectic phase diagrams and two-phase microstructure formation include steel, copper alloys (Cu-Zn, Cu-Sn, and Cu-Al), Ni-based superalloy, rare earth ferromagnet (Co-Sm-Cu and $\mathrm{Nd}-\mathrm{Fe}-\mathrm{B}$ ), and high $\mathrm{T}_{\mathrm{C}}$ superconductors such as $\mathrm{YBa}_{2} \mathrm{Cu}_{3} \mathrm{O}$ [1]. To obtain optimum properties in these materials, which are governed by the microstructure, a correlation between the processing conditions and microstructure is required [2, 3]. (2) Most metallic alloys in the single phase region often form two-phases: stable and metastable phases. This is common during the rapid solidification of alloys. One of the critical aspects is the importance of both the nucleation and competitive growth of the two phases in establishing the microstructure. Specifically, once a given phase nucleates, the conditions of the surrounding liquid change and may make it possible for the second phase to nucleate. In fact, the dynamics of growth and the repeated nucleation of the phases are common in the formation of complex microstructures. The fundamental aspects of such complex microstructure evolution can not be examined quantitatively under rapid solidification conditions. However, the solidification of alloys in the two-phase region of peritectic systems provides an excellent opportunity to isolate repeated nucleation and morphological evolution 


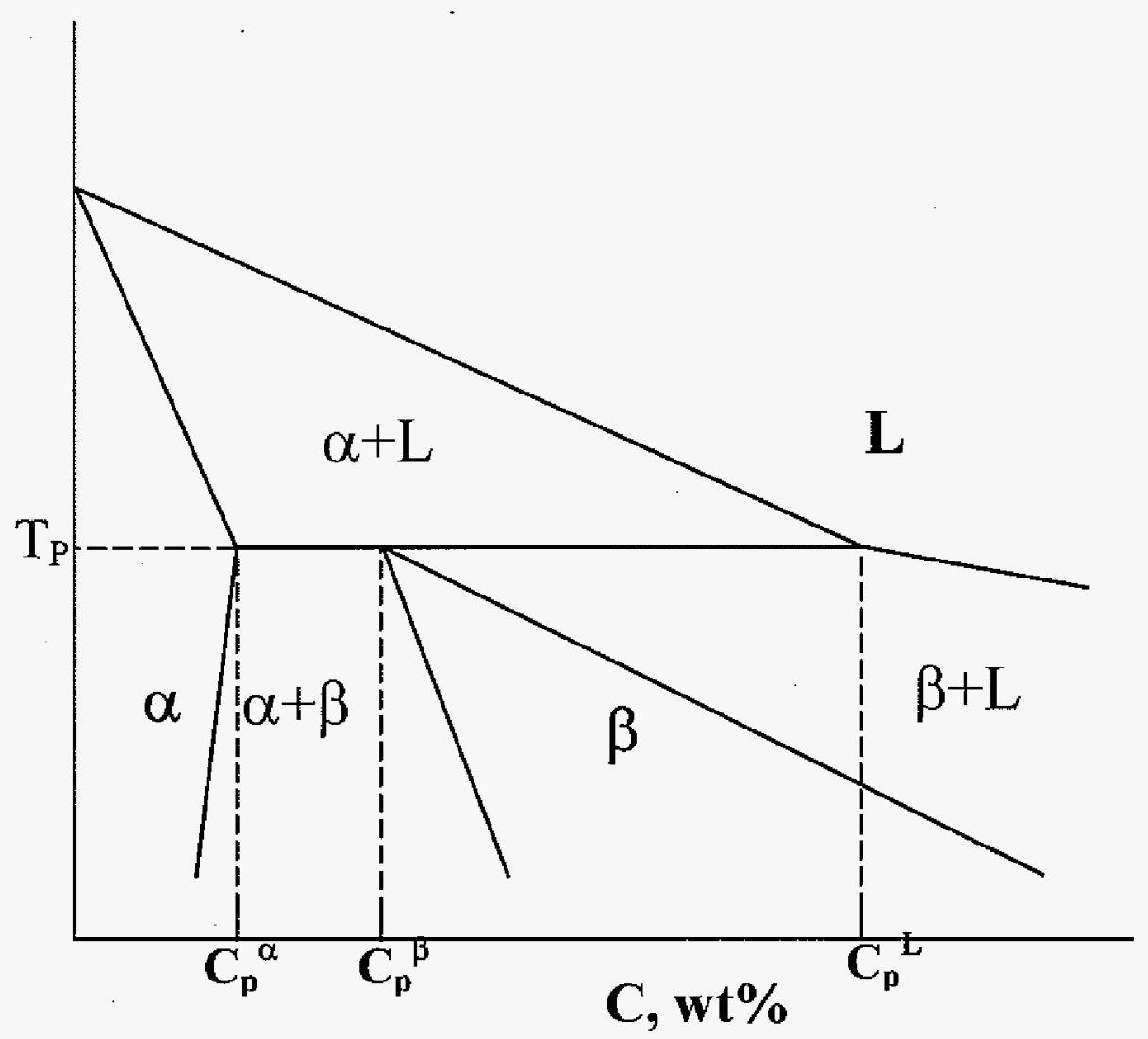

Fig. 1 A typical phase diagram of a peritectic system. 
though the process of growth competition between the primary and the peritectic phases.

The ground work for the study of nucleation and competitive growth was established by Trivedi [4] in his work on the theory of layer formation in peritectic systems. It was shown that the nucleation of the two-phases plays a crucial role in the development of oscillatory structures. It was shown that the nucleation undercooling of the two phases controls the range of compositions over which bands form and the periodicity of the bands. The importance of nucleation is briefly described in the next chapter. The experimental values of nucleation undercooling were found to be very small [5], and the heterogeneous nucleation would require few orders of magnitude lower barrier for nucleation than that predicted by the nucleation theory [6]. The heterogeneous nucleation mechanism will be investigated first.

Once a new phase nucleates on the growing phase, the microstructure evolution is controlled by the complex interaction of the solute field generated by the two phase, and the evolution of the morphology can be determined by the competitive growth of the two phases. Such competition has been shown to give rise to partial bands or composite microstructure [5, $7,8,9]$. The model for composite growth is still not established since it is suggested that this structure may or may not form in a coupled manner with a planar two-phase interface [10-14]. Well-controlled experiments are required to verify this conclusion.

The objectives of dissertation are (1) to present critical experiments to establish the mechanism of microstructure formation in peritectic alloys under diffusive growth conditions, (2) to investigate nucleation undercooling of the two phases and explain the results in terms of an existing or new nucleation heterogeneous nucleation model, (3) to understand the basic mechanism for the two-phase composite growth in peritectic alloys. 


\section{Dissertation organization}

Chapter 2, "Background" presents the current understanding of the topics that are relevant to the work presented in the thesis. This includes nucleation and competitive growth, along with the basic ideas on planar front stability, cellular and eutectic growth.

Chapter 3, "Modelling of Microstructure Evolution in Peritectic Systems" was published in the Materials Science and Engineering A. This paper discusses two major microstructures in directionally solidified alloys in peritectic system (in the two-phase region): oscillatory structure and composite structure. Repeated nucleation and growth competition between nuclei and parent phase play a key role to select oscillatory structure: partial band and complete band structure. Composite structure is observed in peritectic alloy and critical condition of composite structure formation is discussed.

Nucleation is examined in chapter 4, "Heterogeneous Nucleation in Peritectic Systems", which will be submitted to Acta Materialia for publication. This paper reports the critical role of nucleation in the development of oscillatory structures and reports measured nucleation undercooling for the two phases. It is found that classical heterogeneous nucleation model predicts more than two orders of magnitude smaller nucleation undercooling. A new mechanism of heterogeneous nucleation at the wall-solid-liquid interface, based on the clustering process of atoms near the wall, is presented.

Chapter 5, "Coupled and Simultaneous Growth in Peritectic alloys", will be submitted to Metallurgical and Materials Transactions. It is shown that coupled growth in peritectic is not observed when solidification is carried out under diffusive growth conditions. However, a steady-state two-phase structure like a eutectic structure forms in a thin $\mathrm{Ta}$ ampoule. It is shown that the finite contact angle at the wall causes the coupled growth to be 
stable under specific growth condition. The theoretical model of rod eutectic is modified to take into account the presence of a finite angle, and the results are in agreement with the experimental observations.

The general conclusions of the research are given in Chapter 6 with an emphasis on the important contributions to the field made by this research work.

The appended result for convection effects on the diffusion coefficient is presented in appendix 1 .

\section{References}

1. H. W. Kerr and W. Kurz, Int. Mat. Rev., 41(4) (1996) 129-164.

2. M. C. Flemings, solidification Processing, McGraw-Hill, New York, 1974.

3. W. Kurz and D. J. Fisher, Fundamentals of Solidification, Trans Tech Publication Ltd., Switzerland, 1998.

4. R. Trivedi, Metall. Mat. Trans. A, 26A(6) (1995) 1583-1590.

5. R. Trivedi and J.S. Park, J. Cryst. Growth, 235(1-4) (2002) 572-588.

6. R. Trivedi, Scripta Mater, 53(1) (2005) 47-52.

7. A. Karma, W.-J. Rappel, B.C. Fuh, and R. Trivedi, Metall. Mat. Trans. A, 29A(5) (1998) $1457-1470$.

8. W. Kurz and R. Trivedi, Metall. Mat. Trans. A, 27A(3) (1996) 625-634.

9. T. S. Lo, A. Karma, and M. Plapp, Phys. Rev. E, 63 (2001) 031504/1-15.

10. M. Vandyoussefi, H. W. Kerr, and W. Kurz, Acta Mater., 48(9) (2000) 2297-2306.

11. S. Dobler, T.S. Lo, M. Plapp, A. Karma, and W. Kurz, Acta Mater., 52(9) (2004) 2795 2808. 
12. J. H. Lee and J. D. Verhoeven, J. Cryst. Growth, 144(3-4) (1994) 353-266.

13. T. S. Lo, S. Dobler, M. Plapp, A. Karma, and W. Kurz, Acta Meter., 51(3) (2003) 599611.

14. W. J. Boettinger, Metall. Trans., 5(9) (1974) 2023-2031. 


\section{CHAPTER 2: BACKGROUND}

Important background information relevant to the topic discussed in this thesis is briefly summarized here, and the following key issues that still remain unanswered are summarized.

(1) Nucleation, specifically heterogeneous nucleation at the wall-solid-liquid junction that is important for the analysis of oscillatory structure formation.

(2) Planar interface growth dynamics that are critical in the formation of banded or oscillatory microstructures.

(3) Cellular growth of the primary phase that is examined for the growth of a composite microstructure.

(4) Convection effects that are important in the design of ampoule size for experiments in which diffusive growth conditions are present.

(5) Banded microstructure formation that shows the important role of nucleation undercooling in the formation of banded structures.

(6) Eutectic growth model, which is modified to take into account the contact angle at the wall to explain coupled growth in a thin Ta ampoule.

\section{Nucleation}

Solidification is a phase transformation process from the liquid to the solid. A decrease in the free energy is required for the transformation of crystallizing units (atoms, molecules, etc) from the liquid to the solid, while free energy is increased for the formation of the solid/liquid interface. Consequently, a sufficiently driving force for the nucleation is 
required to provide required free energy change to compensate for the increase in free energy required for the interface formation. Therefore, a critical undercooling is usually required to induce nucleation.

The nucleation rate $(I)$ with the critical nucleation size, $r^{*}$, is given by

$$
\mathrm{I}=\mathrm{A} \exp \left(-\frac{\Delta G^{*}}{k T_{N}}\right)
$$

Where $\Delta G^{*}$ is the reversible work required to form a critical cluster size of radius $r^{*}$.

\subsection{Driving force for solidification}

Fig. 1 shows a typical peritectic phase diagram and free energy-composition curves at any temperature below the melting temperature. The driving force for solidification of pure substance, A, can be expressed as:

$$
\begin{aligned}
\Delta G_{\mathrm{r}} & =G_{A}^{S}-G_{A}^{L} \\
& =-\frac{L_{A}}{T_{M}}\left(T_{M}-T\right)=-\Delta S_{1} \Delta T
\end{aligned}
$$

Where $G_{A}^{S}$ and $G_{A}^{L}$ are the molar free energy of the solid and the liquid, $L_{A}$ is the latent heat of melting of pure substance, $\mathrm{A}$, and $T_{M}$ is the melting temperature of pure substance, $\mathrm{A}$.

The driving force for solidification of an alloy of composition $X_{0}$ is given by

$$
\Delta G_{v}=\frac{1}{V_{m}}(1-X s)\left(\mu_{A}^{s}-\mu_{A}^{L}\right)+X s\left(\mu_{B}^{S}-\mu_{B}^{L}\right)
$$

Where $X_{\mathrm{S}}$ is mole fraction of $\mathrm{B}, \mu_{A}^{S}$ and $\mu_{A}^{L}$ are chemical potential of $\mathrm{A}$ in the solid and the liquid, and $\mu_{B}^{s}$ and $\mu_{B}^{L}$ are chemical potential of $\mathrm{B}$ in the solid and the liquid. 
When a small volume of the liquid transforms to the solid by fluctuation in composition, the possible composition range of the solid is between $X_{1}$ and $X_{2}$ as shown in

Fig. 1 (b). The maximum free energy change is given by $\Delta G_{V^{M}}^{\text {Max }}[1,2]$.

\subsection{Homogeneous nucleation [3]}

The free energy of the system, Fig. 2 (a), at a temperature below melting temperature, $T_{M}$, is given by

$$
G_{1}=V_{L} G_{V}^{L}
$$

If some atoms cluster in the liquid to form a small solid sphere, the free energy of the system, Fig. 2 (b), should be changed to $G_{2}$ given by

$$
G_{2}=V_{S} G_{V}^{S}+\left(V_{L}-V_{s}\right) G_{V}^{L}+A_{S L} \gamma_{S L}
$$

When the solid forms in the undercooled liquid, the amount of free energy change is given by

$$
\Delta G=-V_{S} \Delta G_{V}+A_{S L} \gamma_{S L}
$$

Where $\Delta G_{V}=G_{L}-G_{S}$, the volumetric free energy change for the liquid to transform to the solid. It is assumed that $\gamma_{\mathrm{SL}}$ is isotropic and the cluster is a sphere of radius, $r$, the free energy change can be written as

$$
\Delta G=-\frac{4}{3} \pi r^{3} \Delta G_{V^{\prime}}+4 \pi r^{2} \gamma_{s i}
$$

The critical condition can be obtained by $\partial \Delta \mathrm{G} / \partial \mathrm{r}=0$, which is given by

$$
r^{*}=2 \gamma_{S L} / \Delta G_{V}
$$

$r^{*}$ is known as the critical nucleus radius. 
When $r<r^{*}$, the system can decrease the free energy change by dissolution of the solid, while for $r>r^{*}$, the free energy of the system can be lowered as the solid grows. The critical free energy change, $\Delta G^{*}$, required to form the critical nucleus size is given by

$$
\Delta G^{*}=\frac{16 \pi \gamma_{S L}{ }^{3}}{3 \Delta G_{V}^{2}}
$$

\subsection{Heterogeneous nucleation}

\section{Heterogeneous nucleation on a flat substrate}

The minimum undercooling required for homogenous nucleation is generally large. However, in practice, the nucleation undercooling is found to be much smaller than that for homogeneous nucleation due to various existing interfaces in the system, such as the interface between the liquid and ampoule wall, between the liquid and the residual particles, the interface between the liquid and gas bubbles trapped in the cracks in the ampoule wall. For heterogeneous nucleation on a flat substrate (Fig. 3) $[3,4]$, the force balance at the triple junction can be expressed as:

$$
\gamma_{w l}=\gamma_{s w}+\gamma_{s l} \cos \theta
$$

Similarly, the total free energy change for heterogeneous nucleation consists of volume contribution and surface contribution, but the latter should be divided into three parts: (i) the creation of the new solid/liquid interface, (ii) the formation of the solid/substrate interface and (iii) the disappearance of the liquid/substrate interface. Therefore, the total energy change for heterogeneous nucleation can be written as:

$$
\Delta G=-\frac{4}{3} \pi r^{3} F_{v}(\theta) \Delta G_{v}+4 \pi r^{2} F_{s}(\theta) \gamma_{s l}+\pi(r \sin \theta)^{2} \gamma_{s w}-\pi(r \sin \theta)^{2} \gamma_{w I}
$$


(a)
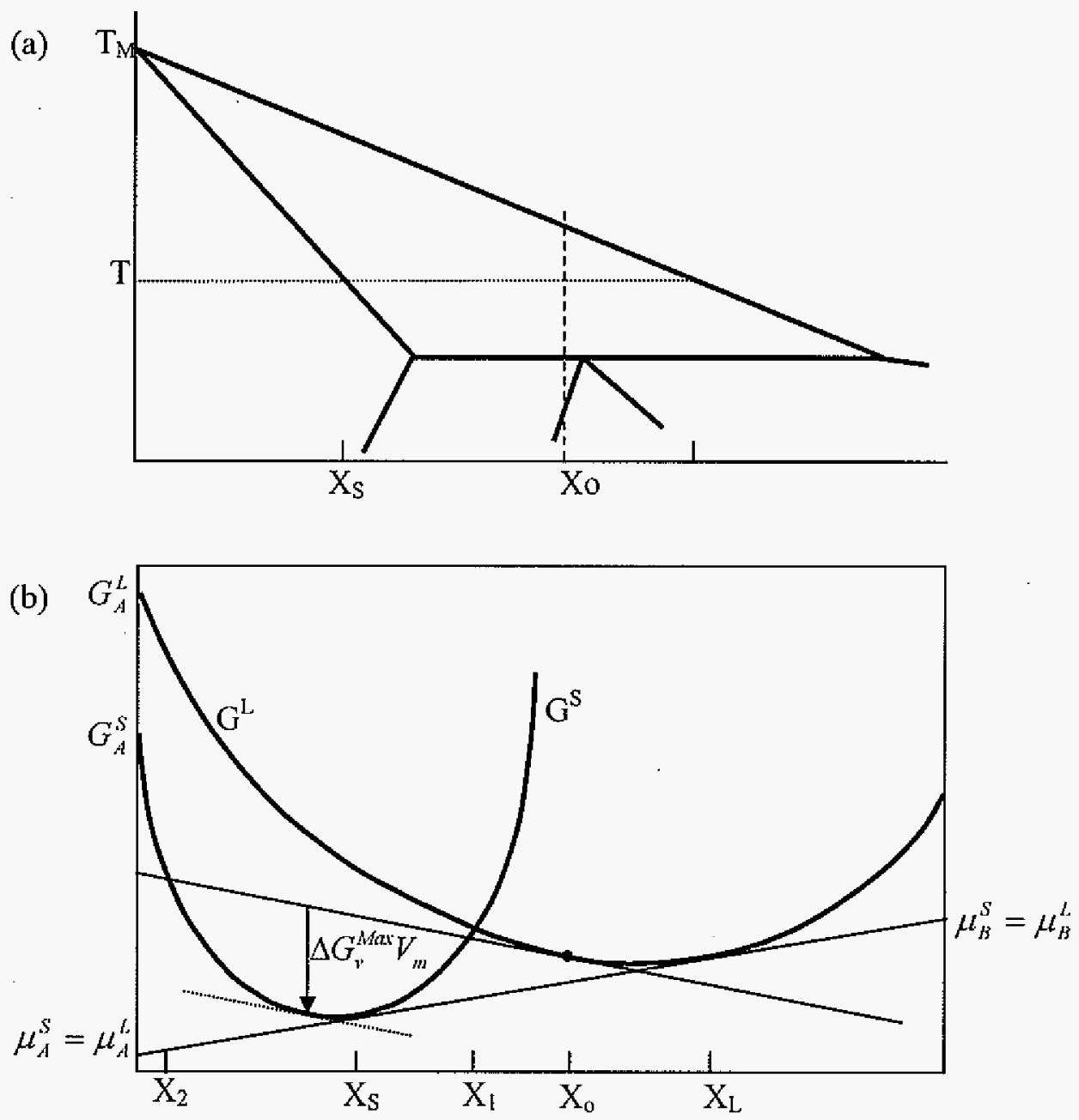

Fig. 1 Peritectic phase diagram and free energy-composition curves. 
The factors $F_{\mathrm{v}}(\theta)$ for the solid volume and $F_{s}(\theta)$ for the solid surface are given by

$$
\begin{aligned}
& F_{v}(\theta)=\frac{1}{4}\left(2-3 \cos \theta+\cos ^{3} \theta\right) \\
& F_{s}(\theta)=\frac{1}{2}(1-\cos \theta)
\end{aligned}
$$

The free energy change for heterogeneous nucleation at a flat surface is given by

$$
\Delta G=\left(-\frac{4}{3} \pi r^{3} \Delta G_{v}+4 \pi r^{2} \gamma_{s t}\right) F_{v}(\theta)
$$

The critical nucleus size is given by $\partial \Delta G / \partial \Delta r=0$

$$
r^{*}=\frac{2 \gamma}{\Delta G_{V}}
$$

and the critical free energy change is given by

$$
\Delta G^{*}=\frac{16 \pi \gamma_{S l}^{3}}{3 \Delta G_{v}^{2}} F_{r}(\theta)
$$

For heterogeneous nucleation, the critical embryo size shows the same radius with homogeneous nucleation under the same undercooling. However, the critical free energy change to overcome the nucleation barrier is smaller than that of homogeneous nucleation by the shape factor, $F_{v}(\theta)$. 

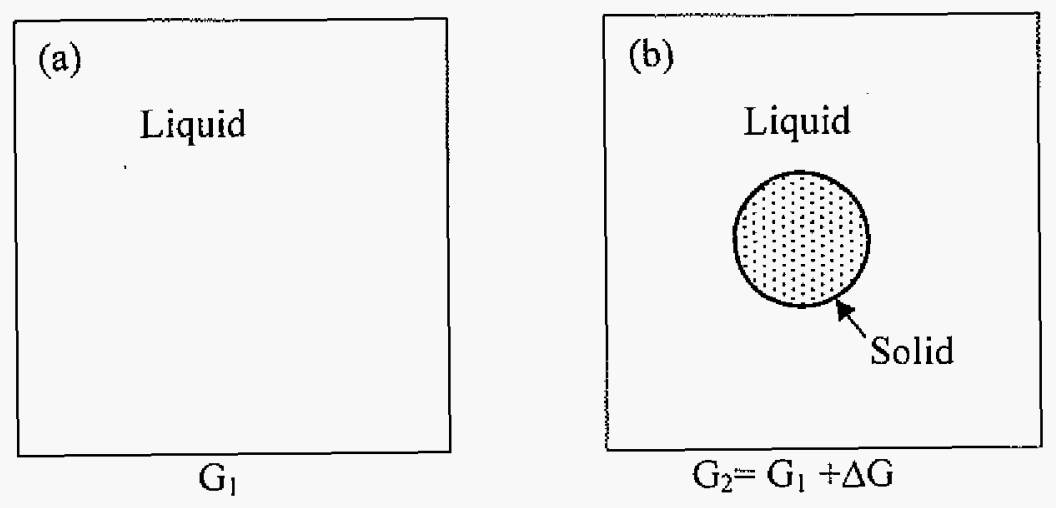

Fig. 2 Homogeneous nucleation.

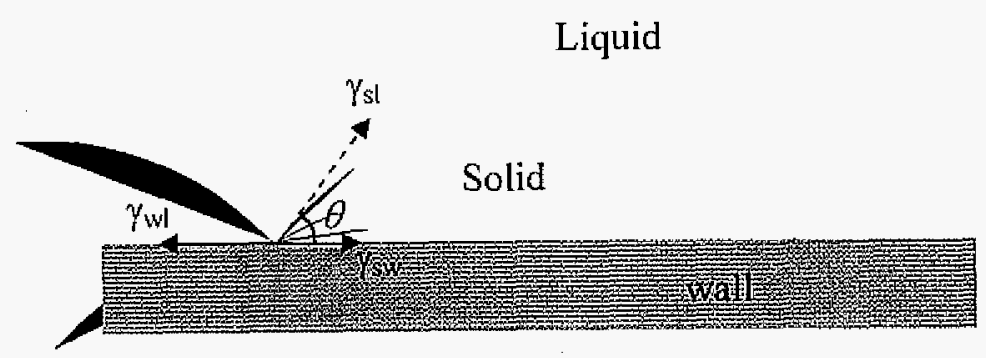

Fig. 3 Heterogeneous nucleation on a flat wall. 


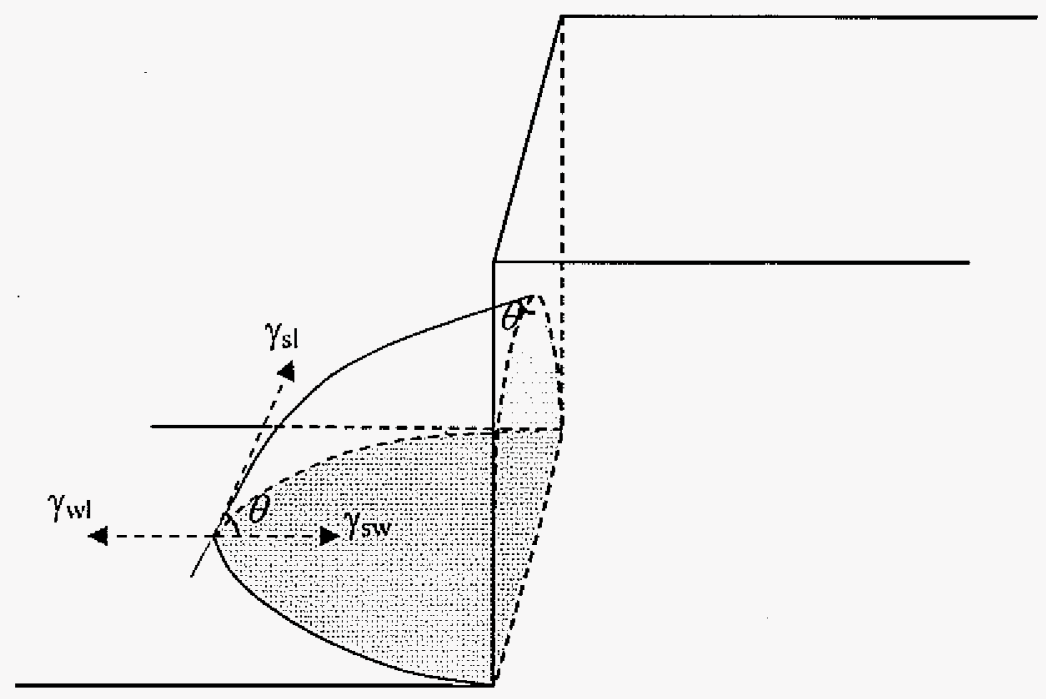

Fig. 4 Heterogeneous nucleation with the same contact angle on a step wall. 


\section{Heterogeneous nucleation on a step substrate}

When heterogeneous nucleus occurs on a step substrate, a nucleus is shown in Fig. 4. Also, assume the same angles for both sides contacting with substrate [5]. When a nucleus occurs heterogeneously at a step substrate, the free energy change was considered by Pound. He showed that the critical nucleus size on a step substrate is independent of nucleation site. Nucleation on the step substrate can occur in a less free energy change than nucleation on the flat substrate since the volume of nucleus on the step substrate is smaller than that on the flat substrate.

Above nucleation site is a very scarce case. However, when the contact angles at the wall side and on the substrate are different, this case can have more chance for nucleation. The critical free energy change required for nucleation was estimated by Trivedi [6]. Fig. 5 shows the schematic nucleation for a angle, $\theta$, contacting with the substrate and a angle, $\phi$, contacting with the wall.

Assuming the nucleus of isotropic interface energy and spherical shape with radius, $r$, the free energy change is given by

$$
\Delta G=\left[-\frac{4}{3} \pi r^{3} \Delta G_{r}+4 \pi r^{2} \gamma_{S L}\right] F(\theta, \phi)
$$

The value of the critical radius of nucleation and the critical free energy change to form a critical nucleus are given by

$$
r^{*}=\frac{2 \gamma_{S t}}{\Delta G_{r}}
$$




$$
\begin{aligned}
\Delta G^{*}=\left[\frac{16 \pi \gamma_{S L}^{3}}{3 \Delta G_{1}^{2}}\right] F(\theta, \phi) & \\
F(\theta, \phi)= & -\frac{1}{4 \pi}\left[\sin ^{2} \phi \cos \phi \cos ^{-1}\left(\frac{\cos \theta}{\sin \phi}\right)+\sin ^{2} \theta \cos \theta \cos ^{-1}\left(\frac{\cos \phi}{\sin \theta}\right)\right] \\
& +\frac{1}{4 \pi}\left[\cos \phi \cos \theta \sqrt{\sin ^{2} \theta-\cos ^{2} \phi}+\cos \phi \cos \theta \sqrt{\sin ^{2} \phi-\cos ^{2} \theta}\right] \\
& -\frac{1}{2 \pi}\left[\cos \phi \cos ^{-1}\left(\frac{\cos \theta}{\sin \phi}\right)+\cos \theta \cos ^{-1}\left(\frac{\cos \phi}{\sin \theta}\right)\right]+\frac{1}{2 \pi} \cos ^{-1}\left(\frac{\cos \theta \cos \phi}{\sin \theta \sin \phi}\right)
\end{aligned}
$$

For $\theta<\pi / 2$, if $\phi>(\pi / 2+\theta)$, nucleation will occur on the substrate.

If $(\pi / 2+\theta)>\phi>(\pi / 2-\theta)$, nucleation will occur at the wall-substrate-liquid junction.

When $\phi<(\pi / 2-\theta)$, no solution is present from Eq. 19 .

For $\theta>\pi / 2$, if $\phi<\theta-\pi / 2$, nucleation will be on the wall-liquid interface.

If $(3 \pi / 2-\theta)>\phi>(\theta-\pi / 2)$, nucleation will occur at the wall-substrate-liquid junction.

When $\phi>3 \pi / 2-\theta$, no solution exists from Eq. 19 .

The above nucleation characters are shown at Fig. 6 with the $(\phi, \theta)$ contact angles showing the different nucleation sites. Fig. 7 (a) shows shape factor value as a function of $\theta$ for a fixed $\phi$ angle. For a fixed $\phi$ angle, a range of $\theta$ angle exists except $\phi=\pi / 2$. The angle range means the possibility of nucleation at w-s-l junction. When the two contact angles have the same value, the shape factor was calculated and compared with the shape factor on the flat surface. The same contact angle is possible when nucleus occurs at the ledge on the surface in cubic structure. The shape factor at the corner has lower value than that on the flat surface under the same contact angle as shown in Fig. 7 (b). In both nucleation sites, a low contact angle has low shape factor value. For nucleation at the corner, smaller free energy 
change for nucleation is required since nucleus at the corner contains smaller volume than that on the flat surface.

The above model is for a nucleus showing different contact angles at perpendicular substrate and wall surface. In general, the angle between the substrate and wall surface is not perpendicular so that we should consider an arbitrary angle between the substrate and wall surface as shown in Fig. 8 [6]. The critical free energy change is given by

$$
\begin{aligned}
& \Delta G^{*}=\left[\frac{16 \pi r_{S L}^{3}}{3 \Delta G_{1}^{2}}\right] F(\theta, \phi, \eta) \\
& F(\theta, \phi, \eta)=-\frac{1}{4 \pi}\left[\sin ^{2} \theta \cos \theta \cos ^{-1}\left[\cot \theta \cot \left(\frac{\eta_{1}}{2}\right)\right]+\sin ^{2} \phi \cos \phi \cos ^{-1}\left[\cot \phi \cot \left(\frac{\eta_{2}}{2}\right)\right]\right] \\
& +\frac{1}{4 \pi}\left[\cos ^{2} \theta \cot \left(\frac{\eta_{1}}{2}\right) \sqrt{\sin ^{2} \theta-\cos ^{2} \theta \cot ^{2}\left(\frac{\eta_{1}}{2}\right)}\right. \\
& \left.+\cos ^{2} \phi \cot \left(\frac{\eta_{2}}{2}\right) \sqrt{\sin ^{2} \phi-\cos ^{2} \phi \cot ^{2}\left(\frac{\eta_{2}}{2}\right)}\right] \\
& -\frac{1}{2 \pi}\left[\cos \theta \cos ^{-1}\left[\cot \theta \cot \left(\frac{\eta_{1}}{2}\right)\right]+\cos \phi \cos ^{-1}\left[\cot \phi \cot \left(\frac{\eta_{2}}{2}\right)\right]\right] \\
& +\frac{1}{8 \pi}\left[\cos ^{-1}\left(\frac{\cos \frac{\eta_{1}}{2}}{\sin \theta}\right)+\cos ^{-1}\left(\frac{\cos \frac{\eta_{2}}{2}}{\sin \phi}\right)\right]
\end{aligned}
$$

For a nucleus with the three arbitrary contact angles, 3-D nucleation map is shown in Fig. 9. The 3-D nucleation map shows a different heterogeneous nucleation site. The shaded tetrahedron represents that nucleation occurs at the wall-substrate-liquid junction. The region A tetrahedron represents the nucleation at the substrate-liquid interface and the region $B$ tetrahedron represents the nucleation at the wall-liquid interface. The region $\mathrm{C}$ and $\mathrm{D}$ represents no heterogeneous nucleation. 


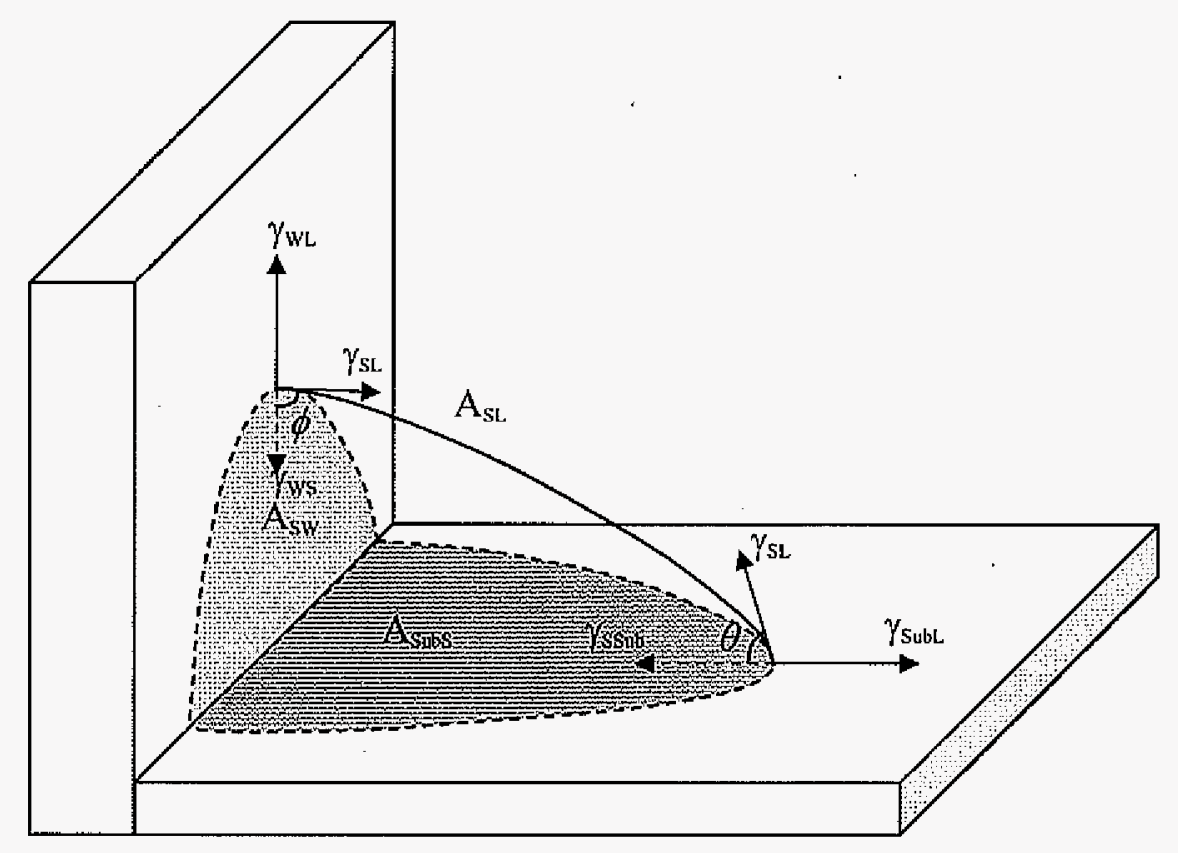

Fig. 5 Heterogeneous nucleation with different contact angles on a step wall.

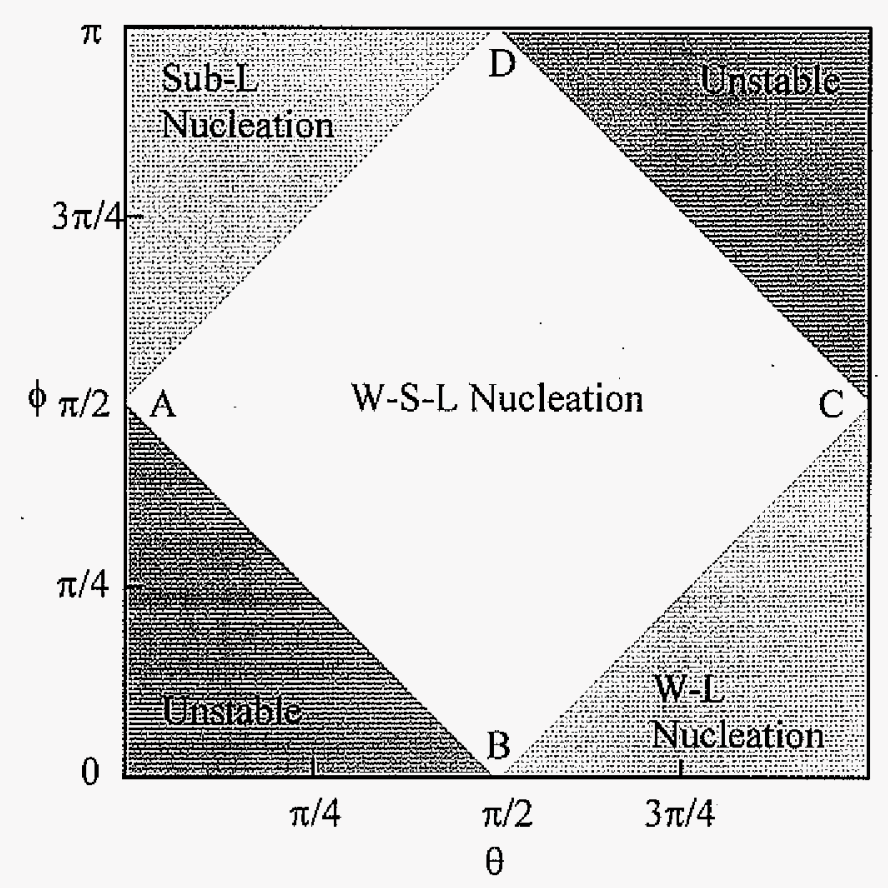

Fig. 6 A nucleation site map under different contact angles [6]. 


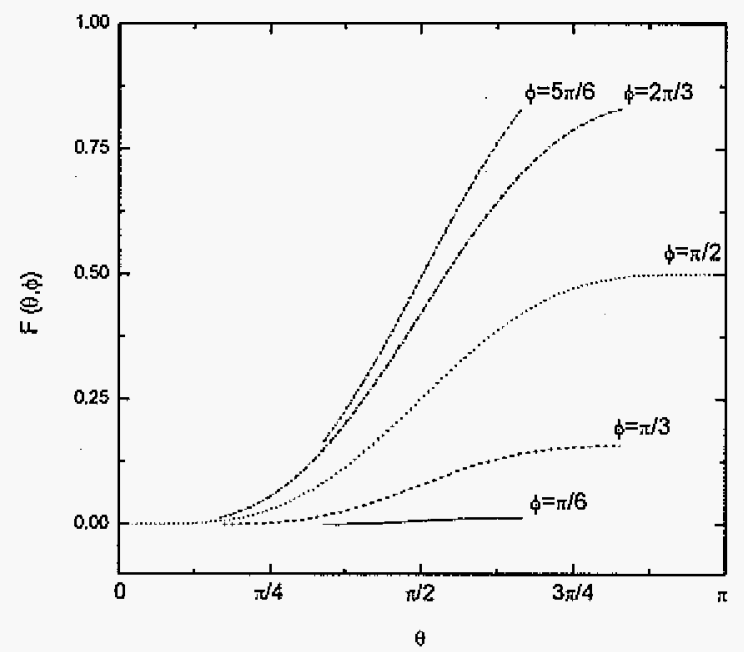

(a)

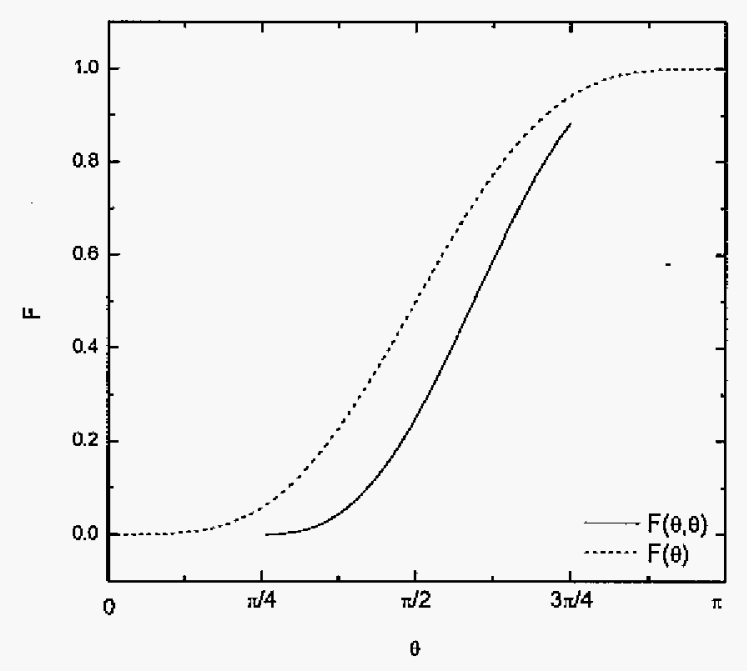

(b)

Fig. 7 (a) Shape factor value as a function of contact angle, $\theta$, under a fixed $\phi$, and (b) shape factor value at the comer for the same contact angle and on the flat surface. 


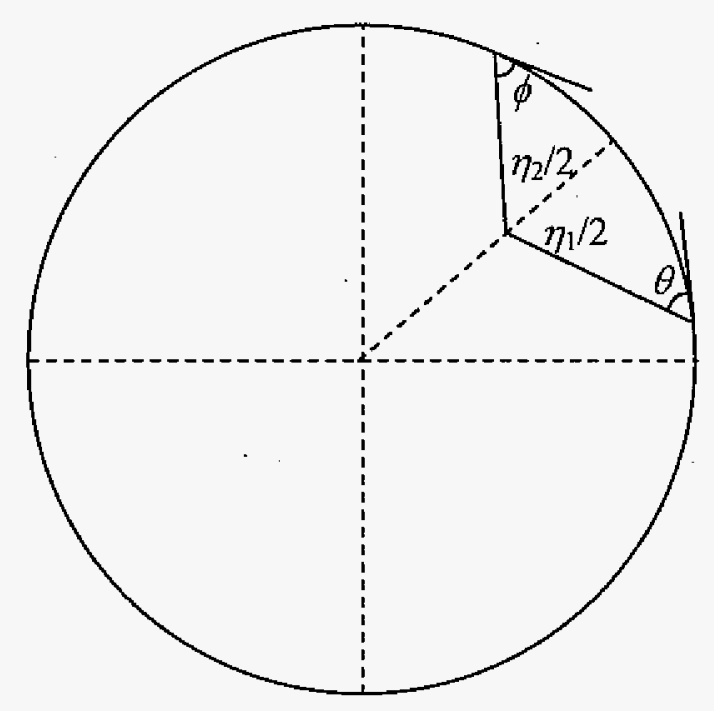

Fig. $8 \mathrm{~A}$ heterogeneous nucleation at a corner with arbitrary angle between the interface and the wall [6].

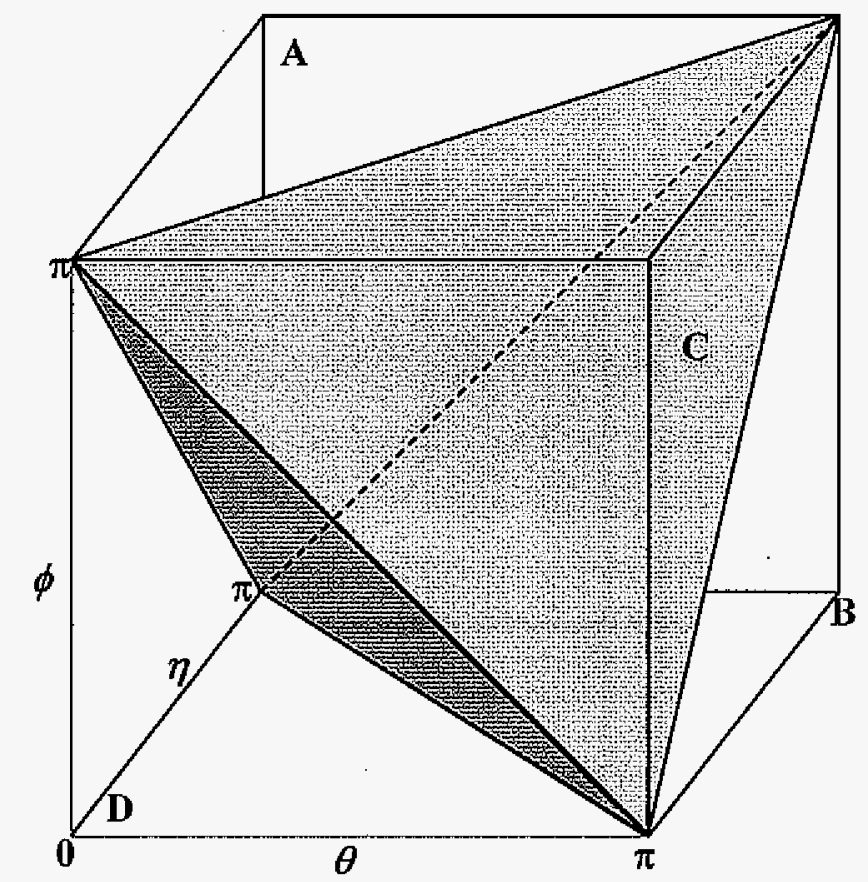

Fig. 9 Heterogeneous nucleation site map at wall-substrate-liquid junction [6]. 
One of the major approximations in the classical heterogeneous nucleation theory is the assumption of equilibrium contact angels. The validity of this assumption requires that the number of atomic layers present in the nucleus is larger than the number of layers that describe the diffuse interface. For very small nucleus size, this is often not satisfied. In addition, nucleation occurs at the interface that is moving at some velocity. The moving interface can trap the cluster so that it might be difficult to build-up large enough cluster required for the critical size.

\section{Interface stability}

The planar interface growth conditions are generally considered for the formation of banded microstructure in the peritectic system. The condition required for the planar interface growth will now be examined.

For pure substances, stability of the $\mathrm{S} / \mathrm{L}$ interface depends on the direction of heat flow. In undercooled melt, the latent heat during growth flows down from the $S / L$ interface to the liquid so that thermal gradient at the $\mathrm{S} / \mathrm{L}$ interface is negative. When a protrusion is formed at the interface, the protrusion can reject heat more effectively to the liquid. As a result, the local growth rate at the protrusion is increased so that the interface can become unstable if the stabilizing effect of interface energy is small. However, in directional solidification, the heat flows from the liquid to the solid and the latent heat during solidification also flows through the solid so that thermal gradient ahead of the interface is always positive. When a perturbation at the $\mathrm{S} / \mathrm{L}$ interface is formed, more heat flows through the protrusion so that it melts back. As a result, the interface is stable and keeps planar shape.

For alloy, when the planar interface grows into the liquid, there is solute pile-up 
ahead of the interface. The solute pile-up decreases the $\mathrm{S} / \mathrm{L}$ interface temperature from $T_{M}+m C_{o}$ to $T_{M}+m C_{o} / k$ when $k$ is less than one, and increases the liquidus temperature with distance, $z$, from the $S / L$ interface. In directional solidification, there is positive thermal gradient arising from the heat flow condition. When thermal gradient is less than the gradient of liquidus line ahead of the interface, $G<m G_{C}$, the undercooled liquid region is present [7]. This is called the region of constitutional undercooling. When a perturbation is formed at the interface, the temperature gradient at the tip of the protrusion is locally increased. However, the concentration gradient at the tip of the protrusion is also increased so that the region of constitutional undercooling can keep preserving and reject more effectively solute into the liquid. The constitutional undercooling criterion shows that a planar interface will be stable when

$$
\frac{G}{\mathrm{~V}}>\frac{\Delta T_{o}}{D}
$$

Where $\Delta T_{o}=m \mathrm{C}_{0}(k-1) / k$, in which $k$ is the solute distribution coefficient, and $m$ is the slope of the liquidus at the solidus temperature.

Constitutional undercooling theory does not explain any scale of the perturbation when the $s / 1$ interface becomes unstable. Also, it explain only thermal gradient in the liquid that keeps interface stable and solute diffusion in the liquid that keeps interface unstable. Mullins and Sekerka [8] investigated the interface stability criterion for planar or non-planar. In their theory, they considered the interface energy effect on the interface stability that keeps interface stable.

In order to analysis, it should be assumed that surface energy is isotropic and the interface is in a local equilibrium, also thermal diffusion and solute diffusion freld is on a 
steady state. When the interface starts out to become unstable, we should consider sinusoidal perturbation by the expression [8-10]:

$$
\mathrm{Z}=\delta \sin (\omega \mathrm{y})
$$

Where $\delta$ is a small amplitude, and $\omega$ is the wave number, $2 \pi / \lambda$.

Interface temperature can be described from solute concentration at interface and interface curvature by the expression:

$$
T_{i}=T_{M}+m C_{i}-\Gamma K
$$

For a planar interface growing with a growth rate, $V$, along the $z$ direction, the steady state solute and thermal fields are given by the equation:

$$
\begin{aligned}
& \nabla^{2} C+\frac{\mathrm{V}}{D} \frac{\partial C}{\partial Z}=0 \\
& \nabla^{2} T_{L}+\frac{\mathrm{V}}{a_{l}} \frac{\partial T_{L}}{\partial Z}=0 \\
& \nabla^{2} T_{C}+\frac{\mathrm{V}}{a_{S}} \frac{\partial T_{S}}{\partial Z}=0
\end{aligned}
$$

Where $\mathrm{C}$ is solute concentration in the liquid, and $T_{L}$ is the thermal field in the liquid and $T_{S}$ is the thermal filed in the solid. $D$ is the solute diffusion coefficient and $a_{L}$ is the thermal diffusion coefficient in the liquid and $a_{S}$ is the thermal diffusion coefficient in the solid. The solution of Eq. 24 can now be obtained by

$$
\begin{aligned}
& T_{i}=T_{o}+a \delta \sin w x \\
& C_{i}=C_{0}+b \delta \sin w x
\end{aligned}
$$

The solution of Eq. 24 are given by 


$$
\begin{aligned}
& C_{L}-C_{o}=\left(\frac{G_{C} D}{\mathrm{~V}}\right)\left(1-e^{-\gamma_{z} / D}\right)+\delta\left(b-G_{C}\right) e^{-\kappa_{C} z} \sin w x \\
& T_{L}-T_{o}=\left(\frac{G_{C} a_{L}}{\mathrm{~V}}\right)\left(1-e^{-\gamma_{z} / a_{L}}\right)+\delta\left(a-G_{L}\right) e^{-w_{L} z} \sin w x \\
& T_{S}-T_{o}=\left(\frac{G_{C} a_{S}}{\mathrm{~V}}\right)\left(1-e^{-\gamma_{z} / a_{S}}\right)+\delta(a-G S) e^{-w_{S} z} \sin w x
\end{aligned}
$$

Unknown values of $a$ and $b$ can be obtained from boundary condition by substituting Eq. 25 and Eq. 26 into Eq. 23.

$$
a=m b-\Gamma w^{2}
$$

The constant $b$ value can obtained from the following Eq. 29.

$$
v(x)=\frac{1}{\Delta H}\left[K_{S}\left(\frac{\partial T_{S}}{\partial z}\right)_{\phi}-K_{L}\left(\frac{\partial T_{L}}{\partial z}\right)_{\phi}\right]=\left\{\frac{D}{C_{\phi}(k-1)}\right\}\left(\frac{\partial C}{\partial z}\right)_{\phi}
$$

The stability condition, $\dot{\delta} / \delta$, can be obtained from $\mathrm{Eq} .29$ by substituting $v(x)=\mathrm{V}+\dot{\delta} \sin w x$

$$
\begin{aligned}
\frac{\dot{\delta}}{\delta}= & {\left[\frac{2 \mathrm{~V} w\left\{w_{C}-\frac{\mathrm{V}}{D}(1-k)\right\}}{\left(K_{S}-K_{L}\right)\left(\frac{2}{K_{S}+K_{L}}\right)\left\{w_{C}-\frac{\mathrm{V}}{D}(1-k)\right\}+2 w m G_{L}}\right] } \\
& \times\left(-\Gamma w^{2}-\frac{K_{S} G_{S}+K_{L} G_{L}}{K_{S}+K_{L}}+m G_{C} \frac{w_{C}-\frac{\mathrm{V}}{D}}{w_{C}-\frac{\mathrm{V}}{D(1-k)}}\right)
\end{aligned}
$$

Since the bracket at the stability condition is always positive, the parenthesis can be written as marginal stability condition. At the marginal stability condition, the first term involving the surface energy has a stabilizing influence for all wave number. The second term has also a stabilizing effect under the condition of positive thermal gradient. The third term shows solute diffusion in the liquid that destabilizes the interface. 
In order for the planar interface to be stable, the stability condition, $\dot{\delta} / \delta$, should be negative for all wave number at a velocity. On the other hand, the interface will be unstable at a range of wave number when the stability condition is positive at a given velocity. The marginal stability can be obtained from the condition, $\dot{\delta} / \delta=0$. At higher wave number than the largest marginal stability, the interface energy becomes important to stabilize the interface. However, at lower wave number than the smallest marginal stability, solute diffusion is required over large distance which is difficult so that interface is stable.

The results of the stability analysis are close to the results obtained from the constitutional undercooling criterion at low velocities. The main result for our study is the condition for the planar interface growth, which is governed by $G / V$ and composition. The planar front will be stable when

$$
\frac{G}{\mathrm{~V}}>\left(\frac{m(k-1)}{D k}\right) C_{o}
$$

This stability condition is then represented by a line on the $G / V$ versus composition plot that will be used to define the regime for the banded microstructure formation.

\section{Cellular growth}

A necessary condition for the formation of stable protrusions on a planar interface is the presence of a constitutionally undercooled zone in the liquid for alloy. If the temperature gradient ahead of the interface is steeper than the critical, $\frac{d T_{q}}{d z}>\left.\frac{d T_{L}}{d z}\right|_{z=0}$, the protrusion will melt back $[11,12]$. If the temperature gradient ahead of an initially planar interface is gradually reduced below the critical value, the first stage in the breakdown of the interface 
may be the formation of a cellular structure. Cellular structure can be observed only in directional solidification. The tip temperature of cell is given by

$$
T_{i}=T_{L}-\Delta T=T_{L}-\left(\Delta T_{S}+\Delta T_{C}+\Delta T_{K}\right)
$$

Where $T_{L}$ is liquidus temperature, $\Delta T_{S}$ is solutal undercooling, $\Delta T_{C}$ is curvature undercooling which is negligible for large tip radius in cellular structure, and $\Delta T_{K}$ is kinetic undercooling which is negligible at a low growth rate. Tip temperature is considered by only solutal undercooling in cellular structure. Concentration at the cell tip is decided by solute gradient at intercellular region, assuming there is no solute gradient in lateral direction at intercellular region. Solute gradient at intercellular region is given by $G_{C}=G / m[13]$.

The concentration in the liquid ahead of tip is approximated as:

$$
C_{L}^{*}=C_{o}-\frac{D G}{\mathrm{~V} m}
$$

This equation gives a good estimate for systems with very small solute distribution coefficient. The tip temperature of cellular structure is then obtained as:

$$
T_{i}=T_{L}-\frac{D G}{\mathrm{~V}}
$$

Tip temperature is increased with increasing growth rate and tip temperature is decreased with increasing thermal gradient. Therefore, cellular microstructures are only stable for a certain range of temperature gradients or velocities. At sufficiently low temperature gradients or high velocities, the cells are observed to develop secondary arms: dendritic structure. Tertiary arms develop at a further lower temperature gradients or higher velocities. 


\section{Convection effects}

Convection effects driven by density discrepancy may fall into two categories depending on the origin of driving force with respect to the gravitation vector: (1) convection by a lateral density gradient, and (2) convection by a vertical density gradient [14].

The vertical density gradients may arise from either thermal convection or a solutal convection. Consider vertical solidification of a pure substance in a tube with radius, $R$. If a thermal gradient is present without a solute gradient, convection will not occur unless a vertical density gradient exceeds a critical limit. The critical value for melt flow is characterized by the Rayleigh number [15]:

Thermal Rayleigh number is given by:

$$
R a_{r}=\frac{g \alpha_{T} G R^{4}}{\nu K}
$$

Where $g=$ gravitational acceleration constant, $\alpha_{T}=$ thermal volume expansion coefficient, $G=$ thermal gradient, $R=$ the sample diameter, $v=$ viscosity, and $K=$ thermal diffusivity. Since $R a_{T}$ can be varied using thin tubes with a range of sample diameters, the condition of generating convection can be changed by changing a sample diameter.

Similarly, the solutal Rayleigh number is given by [15]

$$
R a_{S}=\frac{g \alpha_{S} G_{S} R^{4}}{w D}
$$

Where $D=$ the mass diffusion coefficient, $G_{S}=$ the solutal gradient, $\alpha_{S}=$ the solutal volume expansion coefficient.

In the case where both thermal and solutal gradient are present, such as an alloy, the critical condition of melt flow is a function of both $R a_{T}$ and $R a_{S}$. This is called thermosolutal 
convection.

The lateral-composition profiles at the growing s/l interface have been calculated for the Bridgman furnace in our laboratory. The results for both denser and less dense solute alloys are plotted in Fig. 10 with different times. The convection in the denser solute alloys results in a large solute segregation in the radial direction. This indicates that the microstructure should be different at each radial position on the interface. A disorder of spacing has been observed because the primary spacing depends on the composition and the composition gradient at the tip of the cell or dendrite. However, the solute profile in the less dense solute system is homogeneous over the radial direction except at the immediate region of the ampoule wall. Therefore, the microstructure should be uniform in the liquid with each radial position.

\section{Band structure in peritectic alloys}

A band structure forms at high $\mathrm{G} / \mathrm{V}$ growth condition in hypo-peritectic alloys, in which the primary $(\alpha)$ phase and the peritectic $(\beta)$ phase nucleate and grow alternately. There is a theoretical model for band structure formation, which assumes (i) convection-free condition in the melt, (ii) rapid growth in lateral direction and (iii) constant interface velocity equal to the externally pulling velocity. The model for band structure is illustrated in Fig. 11 [16-20]. When a hypo-peritectic alloy, $\mathrm{C}_{0}$, is directionally solidified under planar interface growth condition, the alloy should be initially solidified with the $\alpha$-phase at point a. The solidified $\alpha$-phase rejects solute into the liquid to form boundary layer, and the $\alpha / 1$ interface temperature continuously decreases with growth proceeding. Before the $\alpha$-phase reaches at the steady state growth condition, the $\beta$-phase can nucleate at a temperature below peritectic 
temperature as shown in the point $b$ in Fig. 11. When the $\beta$-phase forms, the liquid composition follows the liquidus line of the $\beta$-phase. Since the steady state condition of the $\beta$-phase is present above peritectic temperature, the $\beta / 1$ interface temperature increase with growth after the $\beta$-phase nucleates. If the interface temperature of the $\beta$-phase growing at the steady state condition is higher than the nucleation temperature of the $\alpha$-phase, the $\alpha$-phase can nucleate. After the $\alpha$-phase forms, the liquid composition ahead of the growth interface follows the liquidus line of the $\alpha$-phase. The repetition of bcdeb shows banding cycles. The shaded composition and temperature rectangle shows the banding structure window in the hypoperitectic region. At a composition below the composition window, the $\alpha$-phase can grow at the steady state condition and the $\beta$-phase can grow at the steady state condition at a composition above the composition window [16].

\section{Eutectic structure}

Eutectic structure is defined by structurally coupled growth of two phases and thermodynamically the existence of equilibrium state of three phases at eutectic temperature: $\alpha$-phase; $\beta$-phase; liquid. Coupled growth requires the lateral transfer of solute through the liquid below eutectic temperature. Undercooling below eutectic temperature is required for solute transfer as shown in Fig. 12. Below eutectic temperature, solute concentration discrepancy exists in the liquid ahead of $\alpha$-and $\beta$-phase and drive the lateral transfer of solute in the liquid.

The undercooling is the discrepancy between the interface temperature, $T_{i}$, and the eutectic temperature, $\mathrm{T}_{\mathrm{E}}$ which is given by 


$$
\Delta T=T_{E}-T_{i}=\Delta T_{S}+\Delta T_{C}+\Delta T_{K}
$$

Where $\Delta T_{S}$ and $\Delta T_{C}$ are the solutal and the capillary undercooling. $\Delta T_{K}$ is kinetic undercooling which is negligible at a low growth rate. Composition and curvature at the interface of both phases vary locally. In order to obtain average undercooling of the interface, average composition in the liquid ahead of the interface and average curvature of the interface was considered by Jackson and Hunt [21]. Average solutal undercooling and average curvature undercooling are given by

$$
\begin{aligned}
& \Delta \bar{T}_{S}=m\left(C_{E}-\bar{C}\right) \\
& \Delta \bar{T}_{C}=\Gamma K
\end{aligned}
$$

Where $\vec{C}$ is average composition in the liquid ahead of the interface, $\Gamma$ is Gibbs-Thomson coefficient, and $K$ is average curvature.

Jackson and Hunt [21] solved the diffusion problem using the Laplace equation to obtain average composition in the liquid ahead of the $\alpha$ - and the $\beta$-interface, assuming interface shape is planar. In addition, they assumed that interface temperature of both phases was equal and the undercooling was small so that the composition of both phases was corresponding to the equilibrium eutectic temperature. The contact angle at each phase as shown in Fig. 12 is driven from the mechanical balance at the triple point: $\alpha / \beta$ interface; $\alpha / \mathrm{L}$ interface; $\beta / L$ interface. Jackson and Hunt assumed that the interfacial energy is isotropic and obtained the average curvature over the $\alpha / \mathrm{L}$ and $\beta / \mathrm{L}$ interface. The Gibbs-Thomson coefficient and the curvature of interface produce the undercooling, $\Delta T_{C}$. 

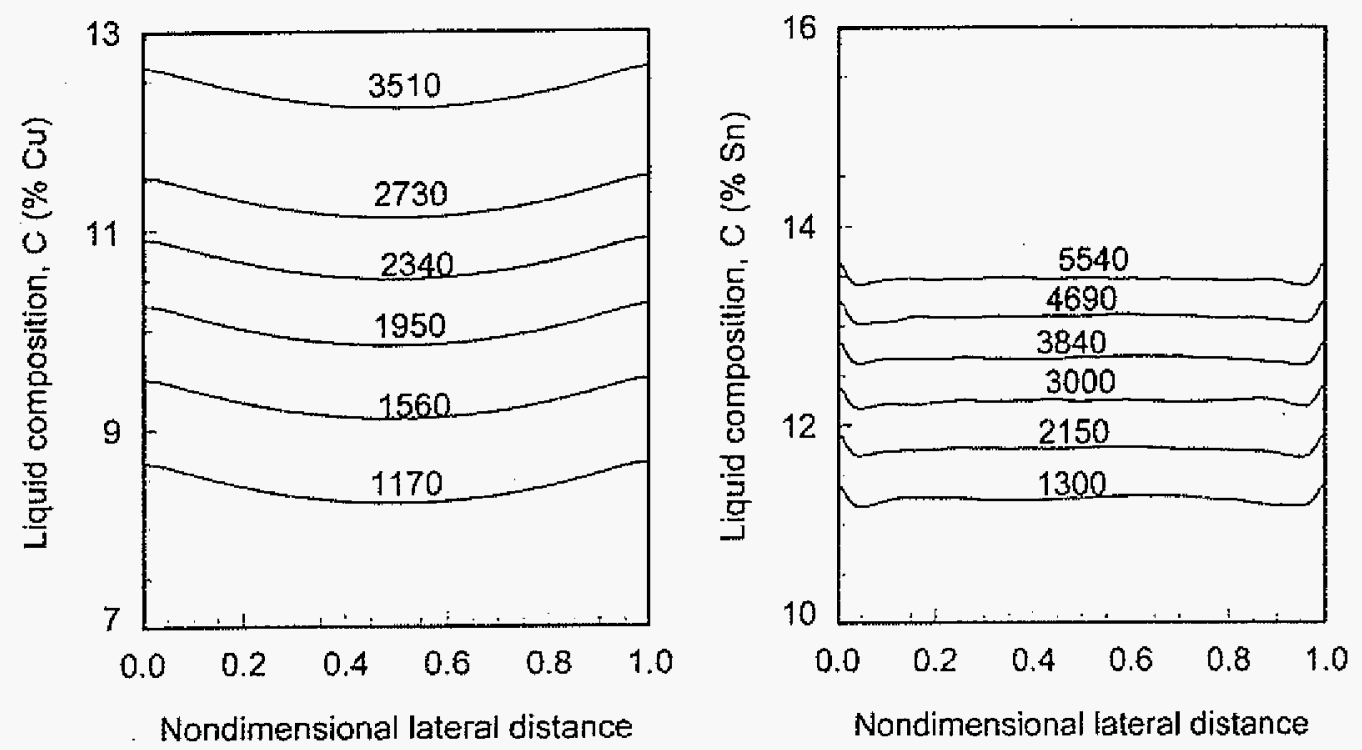

Fig. 10 Lateral interface composition profiles for different instants started from the beginning of solidification.

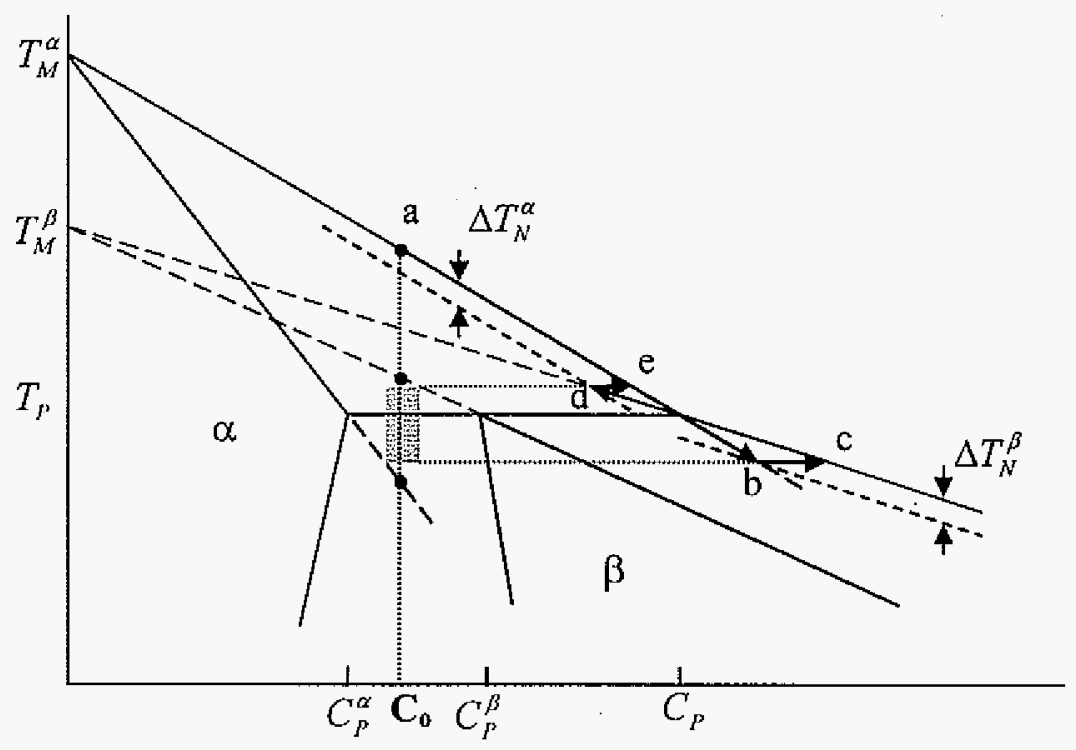

Fig. 11 The schematic drawing of the band structure formation and composition window [16]. 

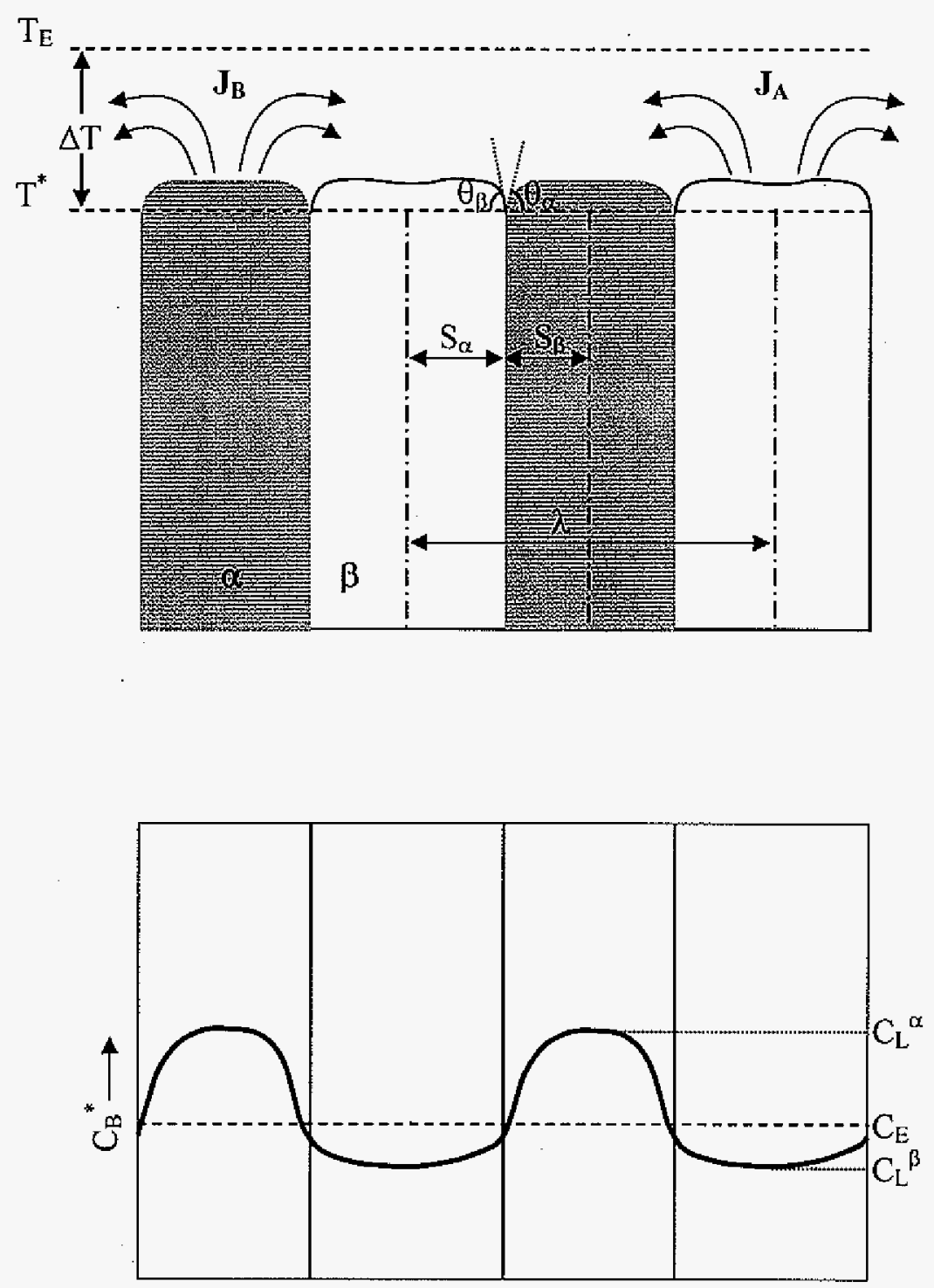

Fig. 12 Eutectic growth and solute profile ahead of interface. 


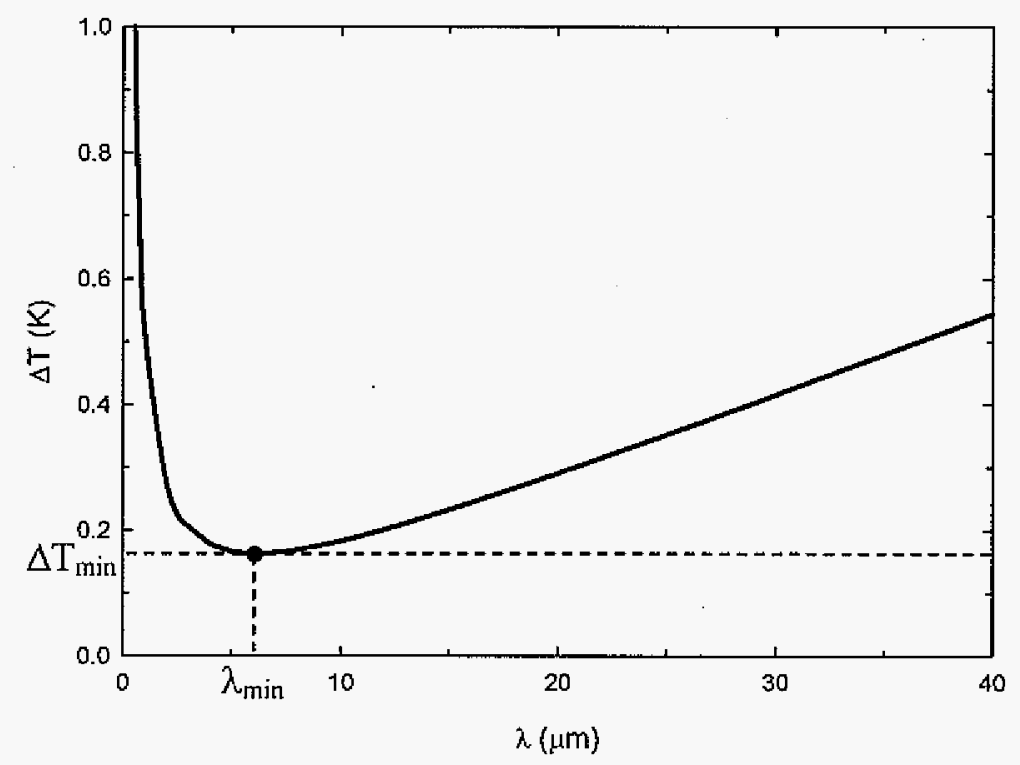

(a)

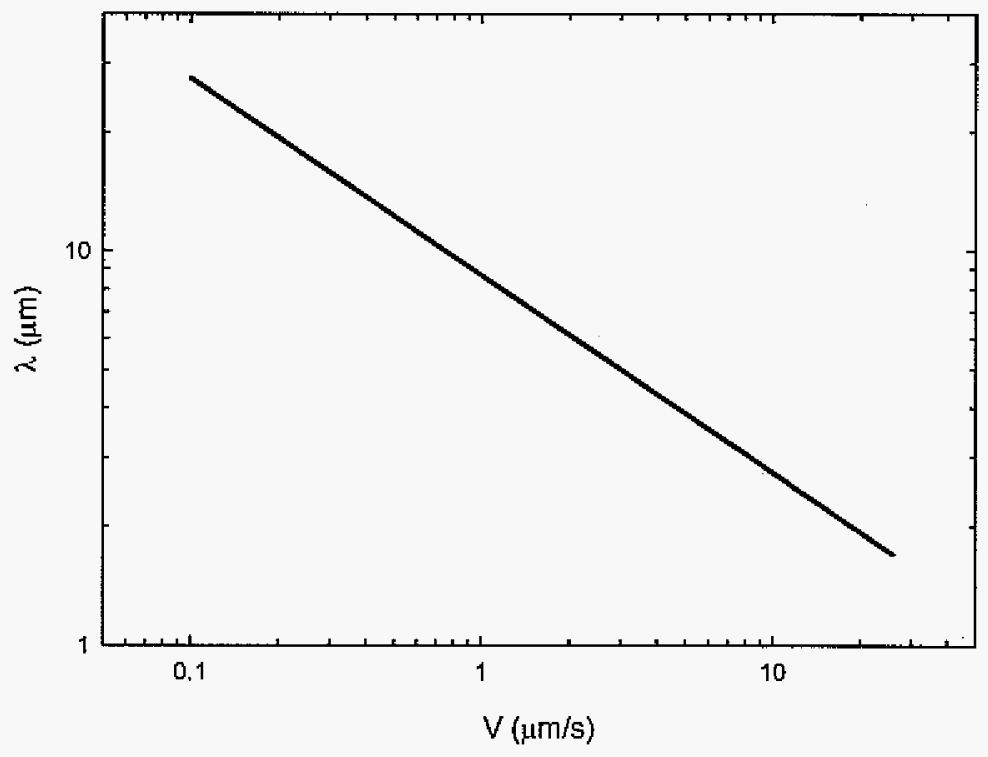

(b)

Fig. 13 (a)Undercooling as a function of eutectic spacing for the lamellar structure at $\mathrm{Al}-\mathrm{Cu}$ alloy and (b) eutectic spacing as a function of growth rate. 
Jackson-Hunt model shows the following relationship among the undercooling, growth rate, and spacing, as

$$
\begin{aligned}
& \Delta T=K_{\mathrm{1}} \lambda V+K_{2} / \lambda \\
& K_{1}=\frac{m P \Delta C_{o}}{f_{\alpha} f_{\beta} D} \\
& K_{2}=2 m \delta\left(\frac{\Gamma_{\alpha} \sin \theta_{\alpha}}{\left|m_{\alpha}\right| f_{\alpha}}+\frac{\Gamma_{\beta} \sin \theta_{\beta}}{\left|m_{\beta}\right| f_{\beta}}\right)
\end{aligned}
$$

Where, $m=\left|m_{\alpha}\right| \cdot\left|m_{\beta}\right| /\left(\left|m_{\alpha}\right|+\left|m_{\beta}\right|\right)$ in which $m_{\alpha}$ and $m_{\beta}$ are the liquidus slop of the $\alpha$-phase and the $\beta$-phase, $\Delta C_{0}$ is the difference between the composition of the $\alpha$-phase and the $\beta$ phase at eutectic temperature. $f_{\alpha}$ and $f_{\beta}$ are the volume fraction of the $\alpha$-phase and the $\beta$ phase. The parameter $\delta$ and $P$ are given by

$$
\begin{aligned}
& \begin{cases}\delta=1 & \text { for lamellar eutectic } \\
P=\sum_{n=1}^{\infty} \frac{1}{(n \pi)^{3}} \sin ^{2}\left(n \pi f_{\alpha}\right) & \text { for rod eutectic } \\
P=2 f_{\alpha} \sum_{n=1}^{\infty} \frac{1}{\left(\gamma_{n}\right)^{3}} \frac{J_{1}^{2}\left(\gamma_{n} f_{\alpha}\right)}{J_{0}^{2}\left(\gamma_{n}\right)} & \end{cases}
\end{aligned}
$$

When the relationship [22-24] between undercooling and spacing calculated by using eq. (39) is shown in Fig. 13 (a), it is clear that the undercooling exhibits a minimum as a function of spacing. The minimum undercooling selects minimum spacing, $\lambda_{\text {min }}$. Eutectic spacing smaller than $\lambda_{\min }$ is destabilized since local depression in the interface occurs, which eliminates a lamellar and increase local spacing. Eutectic spacing larger than $2 \lambda_{\min }$ which is 
called maximum spacing, $\lambda_{M}$, is leading unstable since a pocket forms in the center of the wider phase. The other phase nucleates in the pocket and grows to control the spacing [21]. Recent H. Walker's work [25] for controlling the spacing showed lateral instability before forming the pocket so that the lateral instability adjusts the spacing of lamellar structure.

The relationship between the minimum undercooling and the minimum spacing is obtained by $\frac{\partial \Delta T}{\partial \lambda}=0 ; \mathrm{V} \lambda^{2}{ }_{\min }=\mathrm{C}$. $\lambda$ versus $\mathrm{V}$ relationship is shown in Fig. 13 (b) on the logarithmic scales in which the spacing is decreased linearly as a function of growth rate with $-1 / 2$ slope.

\section{References}

1. B. Cantor and R. D. Doherty, Acta Metal., 27(1) (1979) 33-46.

2. C.V. Thompson and F. Spaepen, Acta Metall., 31(12) (1983) 2021-2027.

3. D. A. Porter and K. E. Easterling, Phase Transformation in Metals and Alloys, Chapman \& Hall, 1992.

4. J. Lothe and G. M. Pound, J. Chemical Physics, 36(8) (1962) 2080-2085.

5. B. K. Chakraverty and G. M. Pound, Acta Metall., 12(8) (1964) 851-860.

6. R. Trivedi, Scripta Mater., 53(1) (2005) 47-52.

7. W. A. Tiller, K. A. Jackson, J. W. Rutter, and B. Chalmers, Acta Metall., 1 (1953) 428-437.

8. W.W. Mullins and R. F. Sekerka, J. Applied Physics, 35(2) (1964) 444-451.

9. J. A. Warren and J. S. Langer, Phys. Rev. E, 47(4) (1993) 2702-2713.

10. R. Trivedi and W. Kurz, Acta Metall., 34(8) (1986) 1663-1670.

11. R. Trivedi, J.A. Sekhar, and J. Mazumdar, Principle of solidification and materials processing, Oxfor\&IBH Publishing Co. PVT. LTD., V.1, 33-66 
12. W. Kurz and D.J. Fisher, Fundamentals of Solidification, Trans Tech Publications Ltd., Switzerland, 1998.

13. V. Laxmanan, Acta. Metall., 33(6), (1985) 1023-1035.

14. R. Trivedi, P. Mazumder, and S.N. Tewari, and R. Trivedi, Metall. Trans. A, 33A(12) (2002) $3763-3775$.

15. J.D. Verhoeven, J.T. Mason, and R. Trivedi, Metall. Trnas. A, 17A(6) (1986) 991-1000.

16. R. Trivedi and J.S. Park, J. Cryst. Growth, 235(1-4) (2002) 572-588.

17. R. Trivedi and W. Kurz, Metall. Trans. A, 21A(5) (1990) 1311-1318.

18. R. Trivedi, Metall. Mat. Trans. A, 26A(6) (1995) 1583-1590.

19. W. Kurz and R. Trivedi, Metall. Mat. Trans. A, 27A(3) (1996) 625-634.

20. A. Karma, W.-J. Rappel, B.C. Fuh, and R. Trivedi, Metall. Mat. Trans. A, 29A(5) (1998) $1457-1470$.

21. K. A. Jackson and J. D. Hunt, Trans. Metall. AIME, 236(8) (1966) 1129-1142.

22. R. Trivedi and W. Kurz, in Solidification Processing of Eutectic alloys edited by D.M. Stefanescu, G.J. Abbaschian and R. J. Bayuzick, The Metallurgical Society, (1988) 3-34. 23. P. Magnin and R. Trivedi, Acta Metall. Mater., 39(4) (1991) 453-467.

24. R. Trivedi, J. T. Mason, J. D. Verhoeven, and W. Kurz, Metall. Trans. A, 22A(10) (1991) 2523-2533.

25. H. Walker, S. Liu, J. H. Lee, and R. Trivedi, Metall. Mat. Trans. A, 38A(7) (2007) 14171425. 


\title{
CHAPTER 3: MODELLING OF MICROSTRUCTURE EVOLUTION IN PERITECTIC SYSTEMS
}

\author{
A paper published in Materials Science and Engineering $A$ \\ R. Trivedi and J.H. Shin \\ Materials and Engineering Physics, Ames Laboratory (USDOE) and \\ Department of Materials Science and Engineering \\ Iowa State University, Ames, IA 50011, USA
}

\begin{abstract}
Theoretical models for directional solidification microstructures are largely developed for steady-state growth conditions. In the two-phase region of peritectic system, a rich variety of two-phase microstructures form in which the two phases often form under non-steadystate conditions and the microstructure evolution depends on the nucleation characteristics of the two phases as well as on the competitive growth of the two phases under dynamical growth conditions. Theoretical models, based on nucleation and diffusive growth, are presented to establish the mechanisms that lead to different microstructure formation in peritectic systems. The critical role played by nucleation in the selection of oscillatory microstructures is discussed, and experimental studies to select different nucleation sites are described. Experimental studies in the Sn-Cd system are carried out in thin capillary samples to minimize fluid flow effects that are dominant in most experiments. Critical conditions for
\end{abstract}


the observation of composite microstructures are evaluated. These results will be presented to support the predictions of the models.

\section{Introduction}

Solidification microstructures of a single phase solid that forms under steady-state growth conditions (cells, dendrites, eutectic, etc.) are reasonably well understood in comparison to other more complex microstructures in which two or more phases form. The two-phase microstructure often forms under intrinsically non-steady-state growth conditions and it is controlled by the competition between the nucleation and growth of different phases. These conditions are often present during the solidification of peritectic alloys, in which the actual microstructure selection process is controlled by a complex interaction between the nucleation process and the growth as well as between the growth of the nuclei and of the preexisting phase under non-steady-state conditions. As a result, a variety of complex microstructures form that are governed by the dynamical conditions at the interface.

Although many studies of peritectic systems have been carried out over the years [1], convection effects were dominant in most experimental studies that strongly influence the microstructure. In this paper we shall concentrate on the directional solidification studies in capillary samples of $0.6 \mathrm{~mm}$ diameter in which fluid flow effects have been found to be negligible in the Sn-Cd system. We shall consider only the microstructures that form within the composition range in the two phase region of a peritectic system in which both the primary $(\alpha)$ and the peritectic phase $(\beta)$ will be present, as shown in Fig. 1. Two distinct mechanisms will be considered that are present at low and high $G D / \mathrm{V}$ values, where $G, \mathrm{~V}$ and $D$ are the thermal gradient, interface velocity and the diffusion coefficient in the liquid, 
respectively. At low GD/V values, a steady state growth of leading cells or dendrites of the primary phase occurs with the peritectic phase forming in the intercellular or interdendritic region that will give rise to a composite structure consisting of two aligned phases behind the interface [2]. This aligned microstructure then transforms to a single $\beta$-phase as the composition, or $G / V$, is increased. At high $G D / V$ values, a variety of periodic microstructures form under intrinsically non-steady-state growth conditions whose evolution and stability are controlled by the nucleation conditions for the two phases as well as by the growth competition between the nucleated and the parent phases $[3,4]$. We shall first briefly describe the simultaneous growth of the two phases at low GD/V ratio, and then focus on to the microstructure formation under high $G D / V$ ratios. The theoretical models will be described in conjunction with the experimental results in the Sn-Cd system.

\section{Experimental procedure}

Directional solidification studies have been carried out in the $\mathrm{Sn}-\mathrm{Cd}$ system for compositions that lie within the two-phase region, i.e. between $C_{p}^{\alpha}$ and $C_{p}^{\beta}$ in Fig. 1. Since convection effects are generally present in most experiments, and these effects significantly alter the microstructure evolution process, Sn-Cd system was selected since the rejected solute (Cd) is heavier than $\mathrm{Sn}$, so that the density gradient in the liquid at the interface is negative. In this case, it has been shown that diffusive conditions can be obtained by directionally solidifying samples in very thin capillary tubes of diameters $<0.8 \mathrm{~mm}[4,5]$. All the experimental studies were thus carried out in fine capillary samples under different growth conditions and compositions.

The basic microstructures in the two-phase region, obtained in capillary samples 
under diffusive growth conditions, are shown in Fig. 2 (a) and 2 (b). When a larger diameter sample, $5.5 \mathrm{~mm}$, is used strong oscillatory convection is present in the system that destabilizes the band formation and gives rise to a complex treelike primary phase that is surrounded by the peritectic phase, Fig. 2 (c). The evolution of this treelike structure has been shown by Mazumder et al. $[6,7]$ to be due to the oscillatory flow present in the large diameter sample. We shall thus restrict our discussion to the microstructure formation in diffusive regime only.

\section{Theoretical modelling and experimental results}

In order to clearly illustrate the complex nucleation and growth dynamics that contro] the microstructure, we shall use a simplified phase diagram in which the liquidus and the solidus lines of the two phases are linear. Also, we shall use simplified analytical expressions for the transient growth process and the interface instability conditions. Once the basic physics is established, more detailed numerical methods can be used for a given phase diagram that relaxes the above assumptions. We shall first consider the composite and banded microstructures, and then discuss more complex microstructure formation process in which the nucleation site, nucleation density and the growth competition need to be considered simultaneously to predict the microstructure. 


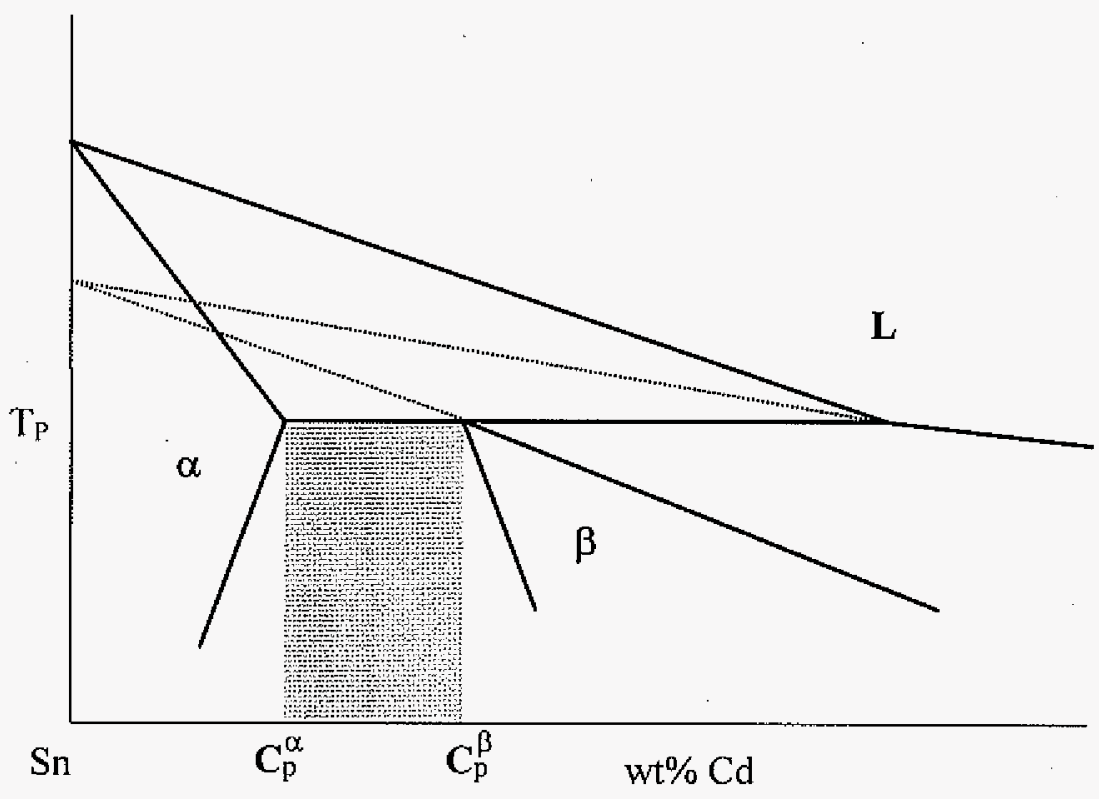

Fig. 1 Phase diagram of the Sn-Cd system. The shaded composition range shows the twophase region examined in this study. 


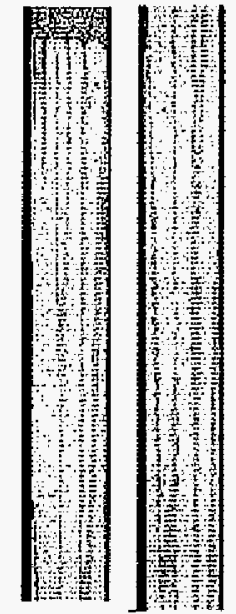

(a)

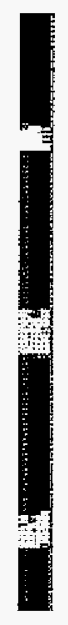

(b)

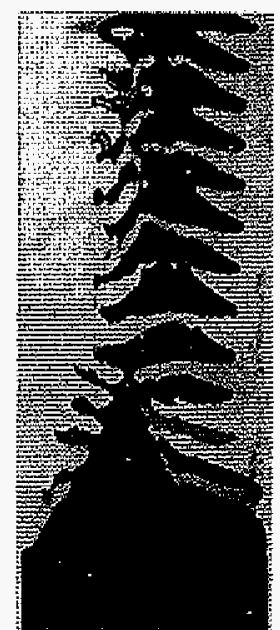

(c)

Fig. 2 (a) A longitudinal cross-section of a composite microstructure in Sn-0.9 wt $\%$ Cd, directionally solidified at $\mathrm{V}=3.6 \mu \mathrm{m} / \mathrm{s}$ and $G=19.5 \mathrm{~K} / \mathrm{mm}$ in thin ampoules of 0.6 $\mathrm{mm}$ I.D. (b) A banded microstructure in thin sample of $0.6 \mathrm{~mm}$ I.D., where the growth is diffusive. (c) A complex treelike primary phase embedded in a peritectic matrix in $5.5 \mathrm{~mm}$ diameter ampoule in which significant convection effects are present. 


\subsection{Composite microstructure}

Consider the growth conditions under low GD/V ratio where the primary $\alpha$ phase is the leading phase with a cellular or dendritic microstructure. Initially we consider growth velocity to be slightly higher such that the $\alpha$-phase tip temperature is above the peritectic temperature. Under a linear imposed thermal gradient, the interface temperature will decrease behind the tip, and when the temperature reaches below the peritectic temperature, $\beta$-phase will nucleate at the $\alpha$-liquid interface. Note that the diffusion in the solid is quite slow so that the peritectic phase will form directly from the liquid rather than by the peritectic reaction. Also, since the peritectic nucleates in the cell groove, only a very small undercooling below the peritectic temperature is required for the nucleation of the $\beta$-phase. Once the $\beta$-phase forms, it will approach the imposed velocity at the steady state growth condition. The precise position of the $\beta$-phase depends on the morphologies of the primary and the peritectic phases.

When the $\beta$-phase forms in the intercellular region where the composition is uniform in the lateral direction, a planar $\beta$-phase will be present when $m_{f} / m_{\alpha}<1$, which is satisfied in peritectic systems so that the $\beta$-interface will be planar when the $\alpha$-interface is cellular [8]. Note that due to the small volume fraction of the $\beta$-phase, and to satisfy the contact angle requirement at the $\alpha$ : $\beta$ :liquid interface, the $\beta$-interface will be curved when the volume fraction of the $\beta$-phase is small. The temperature of the steady-state planar $\beta$-interface can be obtained by examining the flux balance at the $\beta$-liquid interface, and using the Bower et al. [9] approximation for the cellular $\alpha$-tip temperature, the length, $\ell$, between the $\alpha$ and the $\beta$ fronts can be obtained as [8]: 


$$
G \ell=-m_{t \alpha}\left(C_{p}-C_{o}\right)+m_{\beta} C_{p}-\frac{G D}{\mathrm{~V}}\left[1-\frac{m_{\beta}}{m_{\alpha}\left(1-k_{\beta}\right)}\right]
$$

When $\ell$ approaches zero, the above result is not valid since it assumes uniform concentration in the radial direction in the intercellular region. We thus consider the condition when $\ell=0$, or when both the phases are at the same temperature $T_{i}$. Let $\Delta T=T_{i}-T_{p}$. Equating the temperature of the two phases gives

$$
\frac{G D}{\mathrm{~V}}=-m_{a x}\left(C_{P}-C_{o}\right)-\Delta T
$$

Experimental studies in the $\mathrm{Sn}$-Cd system were carried out to examine the relative positions of the $\alpha$ - and the $\beta$-phase at different velocities. Fig. 3 shows the longitudinal and transverse sections of the regions near the advancing front for different velocities. Note that the distance, $\ell$, between the tips of the $\alpha$ - and the $\beta$-phase decreases as the velocity is decreased, as shown in Fig. $4(\mathrm{a})$, and at $\mathrm{V}=3.0 \mathrm{~mm} / \mathrm{s}$, both the fronts are at the same temperature. Such an alignment of the two phases in peritectic system has also been observed previously [10-13]. Transverse sections show that when $l$ is large, the peritectic phase surrounds the primary $\alpha$-cells. However, as the length, $l$, becomes smaller at lower velocities, where the primary spacing increases, the peritectic phase does not uniformly surround the $\alpha$ cells, and the two phases are growing independently. The microstructure is thus significantly different from that of a eutectic, so that the theoretical analysis on the stability of the twophase interface growing isothermally $[10,13]$ may not be general.

Since the tip temperatures were not easy to measure in thin samples, the composition at the cell tip in the $\alpha$-phase was measured, Fig. 4 (b), and the tip temperature was obtained from the composition values, as shown in Fig. 4 (c). The tip temperature of the $\beta$-phase was 
then calculated from the values of $l$ and $G$. Note that when the two phases are at the same temperature the interface temperature is about $0.4 \mathrm{~K}$ above the peritectic temperature. The theoretical value of $\Delta T$ can be estimated from equation (2) by assuming that the undercooling due to interface energy is negligible, which is reasonable for cellular microstructures. However, the precise value of the diffusion coefficient in this system is not known. A good agreement is obtained for $D=1.6 \times 10^{-3} \mathrm{~mm}^{2} / \mathrm{s}$, but independent measurements of the diffusion coefficient are required.

\subsection{Discrete band formation}

We now consider the high $G / V$ ratio condition for which either the leading primary or the leading peritectic phase will grow with a planar interface. The basic concept of Banding Cycle under diffusive growth conditions is illustrated in Fig. 5. According to this model [6], when a melt of initial composition $\mathrm{C}_{\mathrm{o}}$ is solidified, a planar $\alpha$ phase will form initially and grow, and tend to approach steady-state condition corresponding to the $\alpha$-solidus temperature of the alloy. However, before reaching the steady state condition, the peritectic $\beta$-phase will nucleate when the interface composition reaches the value, $\mathrm{C}_{N}^{\beta}$, or the interface temperature reaches, $T_{N}^{\beta}$, which is below the peritectic temperature, as shown in Fig. 5.

We first consider the specific case in which one nucleus forms at the interface and spreads rapidly along the interface to form a band of the $\beta$-phase. The solid and the liquid compositions at the interface will now decrease along the $\beta-\mathrm{L}$ solidus and liquidus lines and tend to approach the steady state solid composition, $C_{0}$, at the $\beta$-solidus temperature. However, before reaching the steady state condition, the primary $\alpha$-phase may again nucleate 

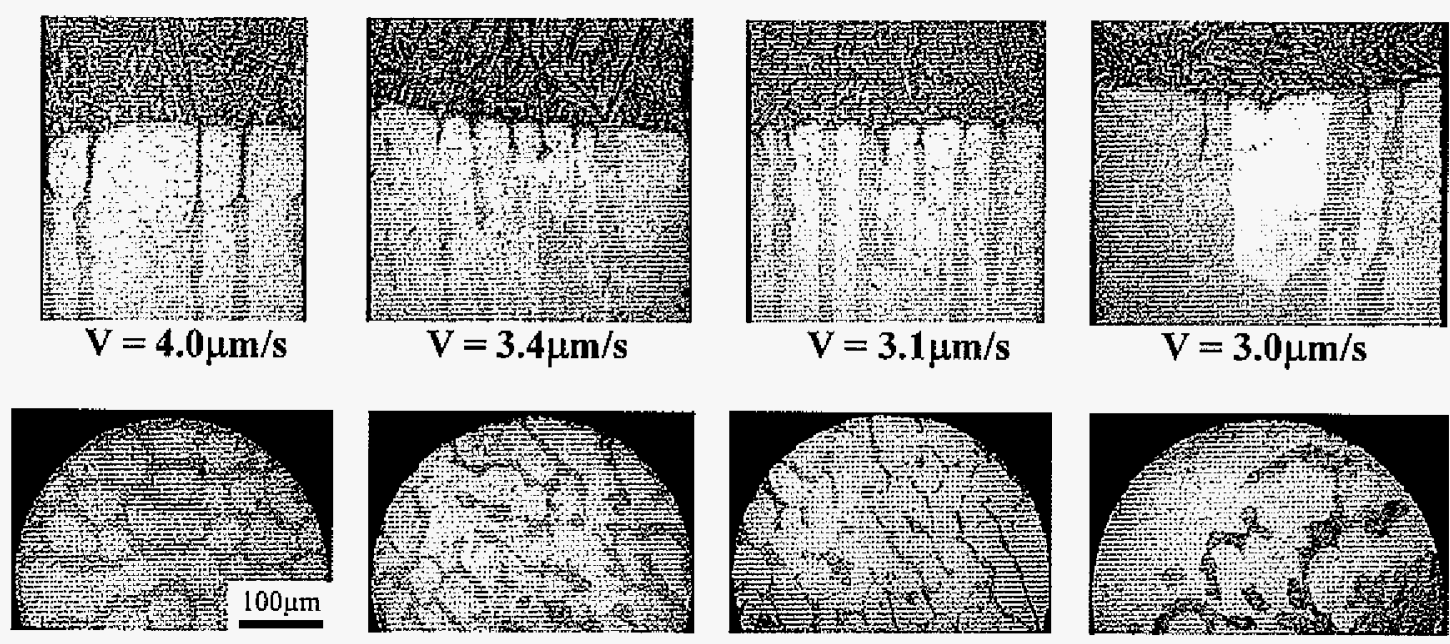

Fig. 3 Experimental results on the microstructure variation with $\mathrm{V}$ in $\mathrm{Sn}-0.9$ wt $\% \mathrm{Cd}$, directionally solidified at $G=19.6 \mathrm{~K} / \mathrm{mm}$ for different values of $\mathrm{V}$. Both longitudinal and transverse sections are shown and the transverse sections were taken about 1.0 $\mathrm{mm}$ below the $\alpha$-tip position. 


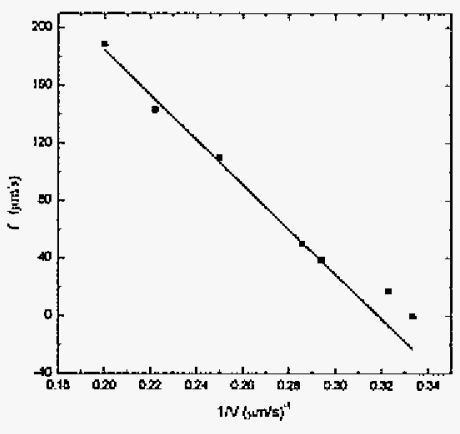

(a)

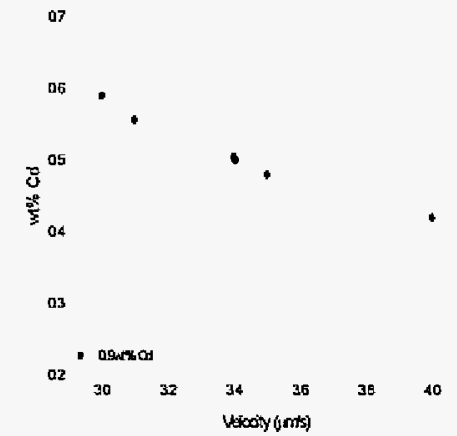

(b)

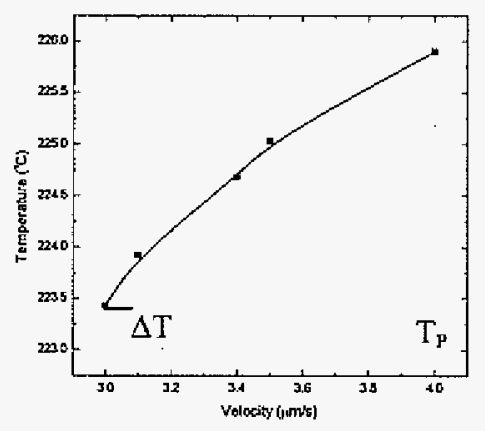

(c)

Fig. 4 The effect of velocity on (a) distance, $l$, between the $\alpha$ and the $\beta$ fronts,

(b) composition in the solid at the $\alpha$-cell tip, and (c) the $\alpha$-cell tip temperature in Sn$0.9 \mathrm{wt} \% \mathrm{Cd}$ alloy, directionally solidified at $G=19.6 \mathrm{~K} / \mathrm{mm}$. 


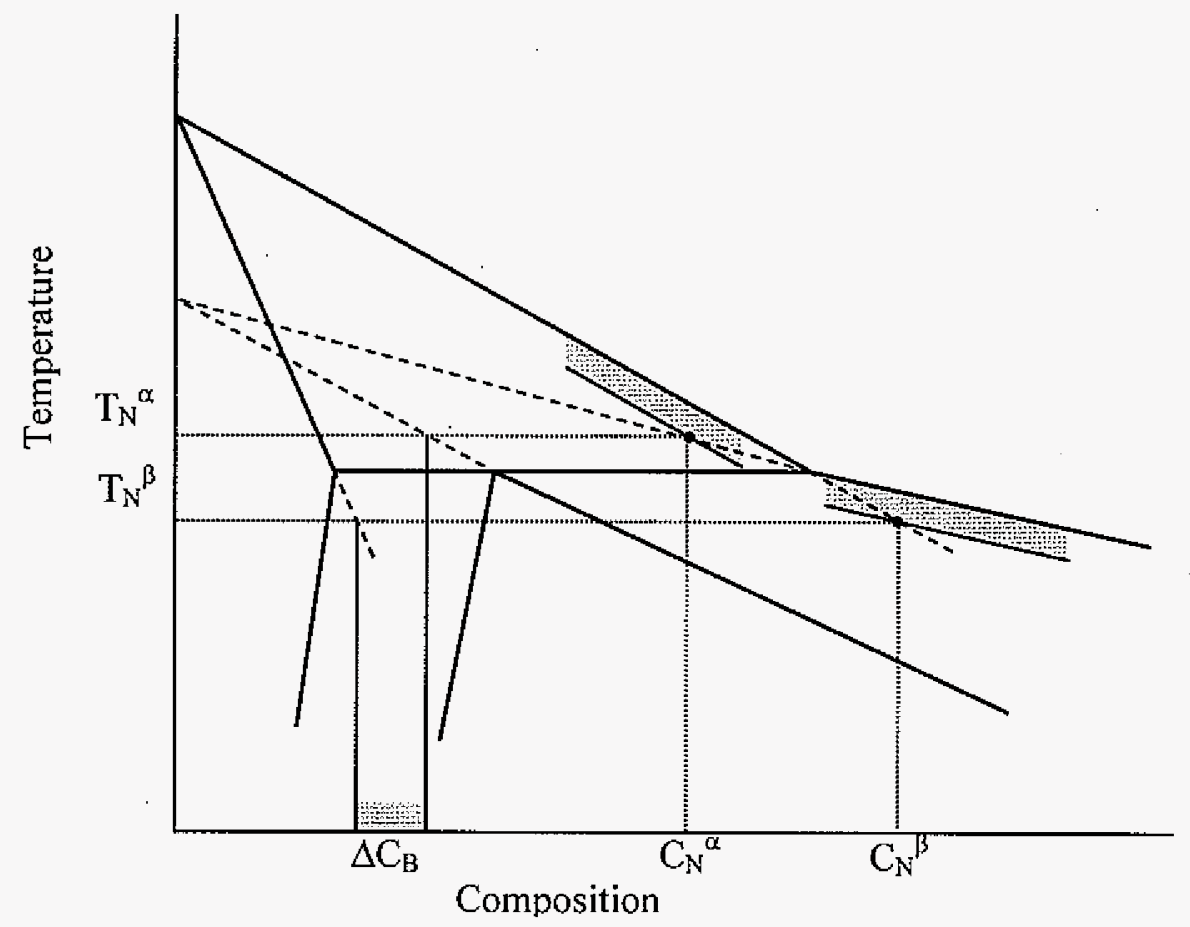

Fig. 5 A schematic drawing of the oscillating composition or temperature cycle for the formation of a banded microstructure. The minimum undercooling required for the nucleation of the $\alpha$ - and the $\beta$-phases is shown by the shaded region below the liquidus lines. $\Delta \mathrm{C}_{\mathrm{B}}$ is the composition range for which diffusive banding is predicted. 
at the solid-liquid interface when the $\beta$ liquidus line intersects the undercooling line for the $\alpha$-phase nucleation, i.e. the composition reaches $C_{N}^{\alpha}$ or the temperature reaches $T_{N}^{\alpha}$. This cycle of alternate nucleation and growth of the primary and the secondary phases will continue leading to a sustained formation of alternate bands of the $\alpha$ - and the $\beta$-phases.

The major predictions of this diffusive banding model are: (a) the banding cycle will operate below and above the peritectic temperature, and (b) the banding window exists only for a narrow composition range, shown as $\Delta \mathrm{C}_{\mathrm{B}}$ in Fig. 5.

We now examine the experimental conditions for the formation of bands. The above discussion of the band formation was for conditions in which both the leading $\alpha-$ and the leading $\beta$-phases would grow as a planar interface, and this is shown as the region 1 in Fig. 6 , in which $G D / \mathrm{V}$ is plotted versus $\mathrm{C}_{0}$. The same mechanism may also be present for experimental conditions below the planar stability line for the $\alpha$-phase since this line is based on the steady-state growth. However, one must also consider dynamic effects such that if the interface composition at which the instability initiates is larger than the composition at which the $\beta$-phase nucleation can occur, the same mechanism as in the region 1 would be present. We may now estimate this condition by considering a simple model that assumes that the interface becomes unstable when the constitutional supercooling condition is reached at the interface, i.e. $m G_{c}=G$. Substituting the value of $G_{c}$ from the flux balance at the interface gives the interface composition in the liquid, $C_{L}{ }^{*}$, at the condition of instability as

$$
C_{L}^{*}=\frac{G D}{\mathrm{~V} m_{*}} \frac{1}{k_{u}-1}
$$


Thus, nucleation of the $\beta$-phase will occur at a planar $\alpha$-interface, and band formation will occur if $C_{t}^{*}>C_{N}^{\beta}$, which gives the condition

$$
\frac{G D}{\mathrm{~V}}>m_{u t} C_{N}^{\beta}\left(k_{a}-1\right)
$$

Since the right had side is constant in a given system, this condition will be satisfied for experimental conditions in region 2 in Fig. 6.

We now consider the case in which the $\alpha$-interface becomes unstable before the condition for the $\beta$-nucleation is reached. As the interface becomes unstable into a cellular pattern, the tip of the cell moves to a higher temperature, whereas the base of the cell moves below the solidus temperature, and the interface composition increases behind the cell tip. Thus, the nucleation conditions for the $\beta$-phase will be reached when the liquid composition at the base of the cell reaches $C_{p}$, since the nucleation undercooling for the $\beta$-phase will be negligible as it nucleates in a narrow liquid groove, as shown in Fig. 6. Once the $\beta$-phase forms, the subsequent microstructure will depend on the growth competition between the $\alpha-$ and the $\beta$-phases. As discussed earlier, the $\beta$-phase will outgrow and become the leading phase when the conditions are to the right of the line $A B$, This leading $\beta$-phase will grow as a planar interface for conditions above the line $O B$, so that within the region 3 in Fig. 6 , banding process will continue. If the experimental conditions were to the left of the line $A B$, the $\alpha$-phase will lead, and a composite structure, shown in Fig. 3, will result. Experiments were carried out in thin samples ( $0.6 \mathrm{~mm}$ diameter) of $\mathrm{Sn}-\mathrm{Cd}$, and banded microstructures were observed in all the three regions. A detailed study of compositional variation in bands was carried out by Trivedi and Park [4], who determined the critical compositions at the $\alpha: \beta$ 
interface to determine the nucleation undercooling. They found $\Delta T_{N}^{\alpha}=0.2 \mathrm{~K}$ and $\Delta T_{N}^{\beta}=1.0 \mathrm{~K}$. They also characterized the nucleation site as the wall:liquid: $\alpha$ triple junction, and found only one nuclei to form and grow. These results show that nucleation conditions can be precisely characterized, and the nucleation undercooling can be measured accurately. In fact, if one can isolate nucleation sites such that nucleation can occur at different specific heterogeneous sites, a detailed understanding of the nucleation process in a given system can be obtained from well-controlled directional solidification experiments. We shall now briefly examine the nucleation sites and show how experiments can be designed to select each of these sites as the preferred nucleation site.

\subsection{Nucleation characteristics}

So far we have assumed the nucleus of the new phase to form and spread and not considered the effect of nucleation site that also influences the microstructure formation. The nucleation of the new phase, (i.e. $\beta$-phase) can occur at the interface-liquid-wall junction or at the interface-liquid position, at the wall-liquid junction or in the liquid ahead of the interface. The selection of the nucleation site has recently been examined by Trivedi [14], who showed that the selection depends on the value of three contact angles, $\theta, \psi$, and $\eta$, shown in Fig. 7 (a). A detatled expression for the free energy change for nucleation at these sites has been developed [14], and the results were presented in terms of a nucleation map. A three-dimensional nucleation map in terms of these three contact angles is shown in Fig. 7 (b), in which the different regions of preferred nucleation sites are given by five tetrahedrons. The central tetrahedron, shown shaded in Fig. 7 (b), is the regime in which nucleation will be 


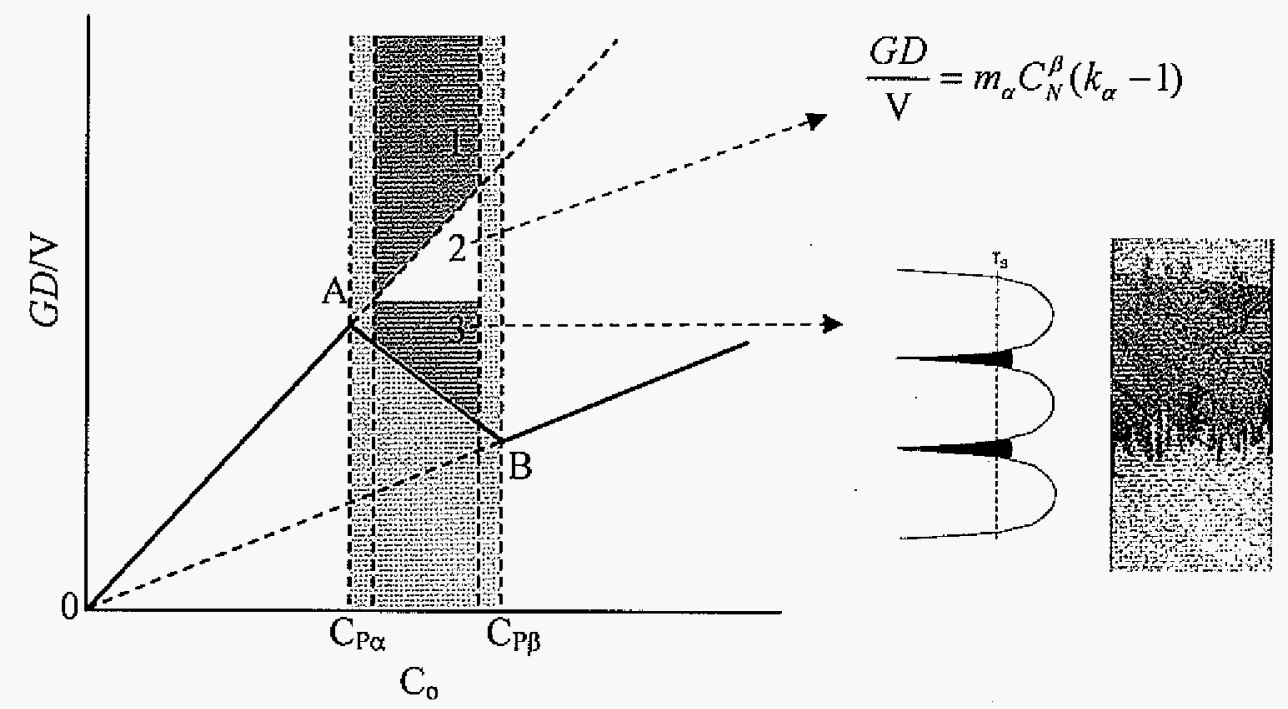

Fig. 6 A schematic diagram that shows the region in the $G / \mathrm{V}-\mathrm{C}_{\mathrm{o}}$ space in which band formation can occur. In the region 1 , either of the phases will grow with a planar front. In the region 2 , the $\alpha$-phase will be planar during the transient growth when the $\beta$ phase nucleation condition is reached. In the region 3 , the $\alpha$-phase becomes nonplanar, and the $\beta$-phase will nucleate in the interdendritic region and then become the leading phase, which is illustrated in the sketch and an experimental observation. 
preferred at the wall: $\alpha$; liquid junction. The other four small tetrahedrons show the regimes of nucleation at other sites. The tetrahedron with an apex at A gives the regime of nucleation at the $\alpha$ :liquid interface, whereas the tetrahedron with apex at $\mathrm{C}$ gives regime of nucleation at the wall:liquid interface. The tetrahedra, with apexes at $B$ and $D$, show the regimes in which nucleation can occur only in the liquid ahead of the interface.

The contact angle $\theta$ is fixed for a given alloy system. However, the contact angles $\psi$ and $\eta$ that form at the wall are controlled by the wall material so that different values of these contact angles can be obtained by using different ampoule materials. If the ampoule materials are selected to preferentially nucleate in different nucleation regimes, undercooling required to nucleate at different sites can be obtained through composition measurements.

\subsection{Partial bands due to growth competition}

In the one-dimensional model of discrete band formation, it was assumed that once the new phase forms, it spreads rapidly in the lateral direction. However, this is generally not valid and more complex microstructures form depending on the relative rates of spreading of the new phase and the continuing growth of the parent phase, as shown in Fig. 8. We consider the formation of a nucleus at the one location on the wall, which is equivalent to examining the region between the center of a nucleus and the half way between the neighboring nuclei, which is controlled by the nucleation rate.

As the $\beta$-nucleus grows in the lateral direction, the $\alpha$-phase also grows in the vertical direction. Thus, a complete band of $\beta$ will not occur if the primary phase will grow faster and engulf the nucleated phase. Fig. 9 shows the general behavior of the competition process in 
which the ratio of the normal velocity of the $\alpha$-phase and the lateral velocity of the $\beta$-phase is shown for complete and partial banding. The following important effects need to be considered. (1) As the $\beta$-phase grows laterally, the fraction of the $\alpha$-phase at the interface decreases and the $\alpha$-phase rejects solute over the width $(\lambda)$ of the region that leads to an increase in its growth rate, i.e.; a one dimensional diffusion field changes to a threedimensional diffusion field ahead of the $\alpha$-phase. This effect becomes important when $\lambda$ is below some critical length, $\lambda_{c}$. Note that, at the other extreme, if $\lambda$ becomes too large, $\alpha-$ interface away from the nucleus will advance significantly before the $\beta$-phase reaches that region, so that partial banding will result. However, as the $\alpha$-interface continues to grow, a significantly larger undercooling will be present for the $\beta$-phase nucleation so that another nucleation site may become active, thereby reducing the distance between the nuclei. (2) Since the $\beta$-phase absorbs solute from the boundary layer of the $\alpha$-phase, its relative growth rate is reduced, which could lead to partial banding. This effect becomes critical when the volume fraction of the $\beta$-phase $\left(f_{\beta}\right)$ is small, so that partial bands will always occur for volume fractions below some critical value. (3) As the velocity is increased, the acceleration of the $\alpha$-phase increases, so that the $\alpha$-phase can engulf the $\beta$-phase. This is shown in Fig. 9 in which the ratio of the normal velocity of the $\alpha$-phase to the lateral velocity of the $\beta$-phase is shown as a function of normalized distance. As the velocity becomes larger, a sharp increase in the $\alpha$-phase growth is observed that leads to the partial band formation. Detailed phase-field model of the partial banding is developed by Lo et al. [15] who discuss some of the above critical factors for the band formation. 
Since the nature of the band of formation depends on the nucleation rate, we now consider the effect of several nuclei on the microstructure development. For the case in which the nuclei form uniformly at the $\alpha$-liquid interface, one would observe the microstructure shown in Fig. 10. For the condition which gives rise to partial bands, the microstructure will consist of discrete particles of one of the two phases embedded inside the continuous matrix of the other phase. Note that nuclei are not generally spaced uniformly so that the distance between them will vary and there will be interaction among microstructures originating between different nuclei so that a fairly complex microstructure can develop that can only be examined by numerical modelling.

\subsection{Complex microstructures}

We have only discussed simple cases in which the nucleation and growth dynamics effects are discussed separately. Also, we have considered the composition variation in the growth direction and the velocity of the interface to be constant. In reality, the growth dynamics will alter the composition profile, often showing a complex $2 \mathrm{D}$ or $3 \mathrm{D}$ variation which will change the characteristics of the subsequent bands so that the time evolution of microstructure can be very complex. Experimentally, we find that the simple map, given in Fig. 6 needs to be used with caution since the interface velocity changes and even oscillates during the formation of microstructures.

The complexity of microstructure evolution can be appreciated by considering the formation of a steady-state composite microstructure near the transition condition, just below the line AB in Fig. 6. In this case the steady-state microstructure will indeed be a leading $\alpha$ phase with intercellular $\beta$-phase. However, the interface velocity initially increases from zero 


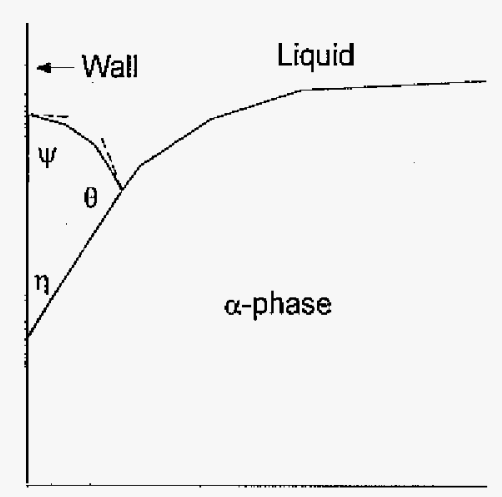

(a)

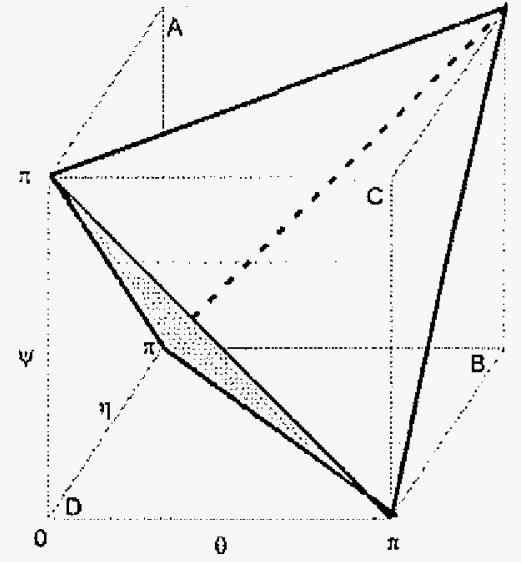

(b)

Fig. 7 (a) An enlarged view of the region near the wall when the nucleation of the $\beta$-phase occurs. The relevant contact angles $(\theta, \psi, \eta)$ are also shown in the figure. (b) A nucleation map in the $(\theta, \psi, \eta)$ space showing the regimes of different nucleation sites. 


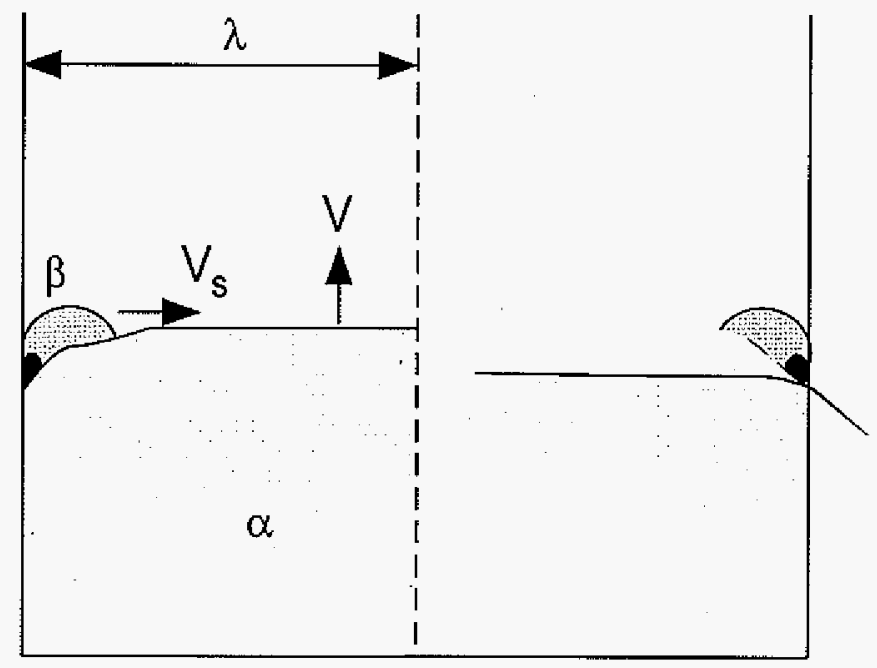

Fig. 8 The growth competition between the nucleated $\beta$-phase and the $\alpha$-parent phase. The distance between the nuclei is $\lambda$, which is controlled by the nucleation rate. 
to the external velocity, so that $G / V$ is very large initially. In this case, band formation will occur in the transient regime. This band formation significantly alters the composition profiles, so that the subsequence microstructure can be quite complex, until the interface velocity approaches the external velocity, and a stable composite structure will be present. This is illustrated in Fig. 11, where few selected sections are shown from the beginning to the steady-state growth. Such complex dynamics requires detailed numerical simulations, although such calculations may not predict since different nucleation characteristics may be present in real systems, i.e. roughness of the wall or impurities in the liquid.

\section{Conclusions}

Basic theoretical concepts have been developed to model the complex microstructure formation in the two-phase region of peritectic systems. Experimental results in thin samples of Sn-Cd alloys have shown the formation of a rich variety of microstructures that depended on the dynamical growth process as well as nucleation characteristics. Some of the fundamental aspects of composite or band or more complex microstructures have been developed to gain an insight into the mechanisms of microstructure formation and the critical role played by nucleation and growth dynamics.

\section{Acknowledgments}

This work was supported by NASA (NCC8-98) and by DOE. This work was carried out at Ames Laboratory which is operated by Iowa State University for the Office of Basic Energy Science, Division of Materials Science, U.S. Department of Energy under Contract No. W7405-Eng-82. 


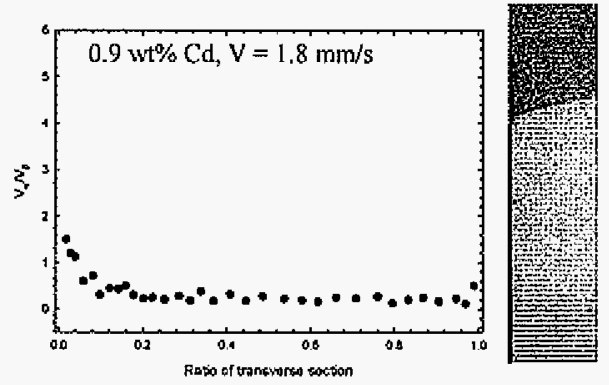

(a)

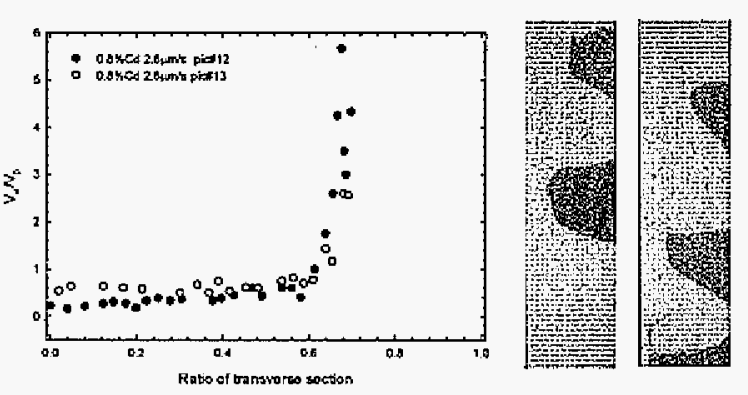

(b)

Fig. 9 The ratio of the normal velocity of the $\alpha$-phase to the lateral velocity of the $\beta$-phase as a function of the fraction of the distance. (a) A complete band in Sn- $0.9 \mathrm{wt} \% \mathrm{Cd}$ at V $=1.8 \mu \mathrm{m} / \mathrm{s}$ and (b) A partial band in Sn-0.8 wt $\% \mathrm{Cd}$ at $\mathrm{V}=2.8 \mu \mathrm{m} / \mathrm{s}$. 


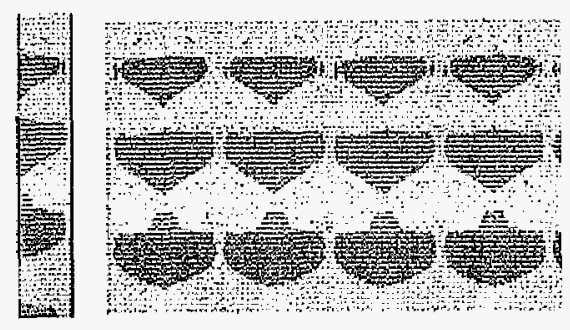

(a)

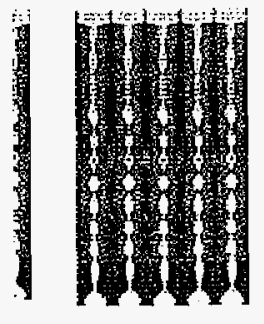

(b)

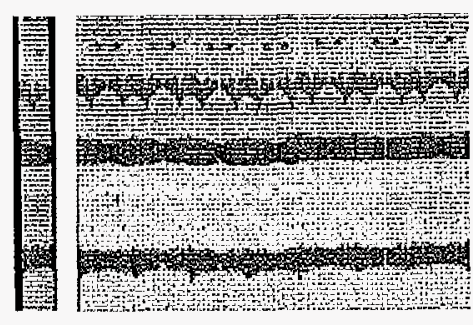

(c)

Fig. 10 Experimental observations of partial bands in thin samples of $0.6 \mathrm{~mm}$ diameter, and the extrapolated effect of multiple nucleation and growth competition on microstructure formation. (a) Partial bands in the Sn- $0.8 \mathrm{wt} \% \mathrm{Cd}$ alloy, $\mathrm{V}=2.8$ $\mu \mathrm{m} / \mathrm{s}, G=19.6 \mathrm{~K} / \mathrm{mm}$, and $\mathrm{d}=0.6 \mathrm{~mm}$. (b) Partial bands in Sn- $0.9 \mathrm{wt} \% \mathrm{Cd}$ alloy, $\mathrm{V}$ $=4.0 \mu \mathrm{m} / \mathrm{s}, G=23.0 \mathrm{~K} / \mathrm{mm}$, and $\mathrm{d}=0.4 \mathrm{~mm}$. (c) Bands in $\mathrm{Sn}-0.75 \mathrm{wt} \% \mathrm{Cd}$ alloy, $\mathrm{V}$ $=2.54 \mu \mathrm{m} / \mathrm{s}, G=19.6 \mathrm{~K} / \mathrm{mm}$, and $\mathrm{d}=0.6 \mathrm{~mm}$. 


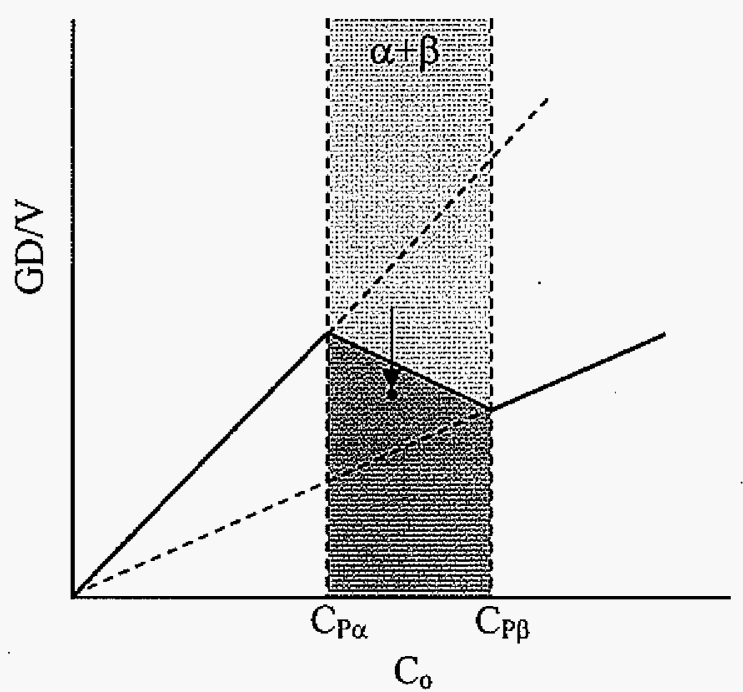

(a)
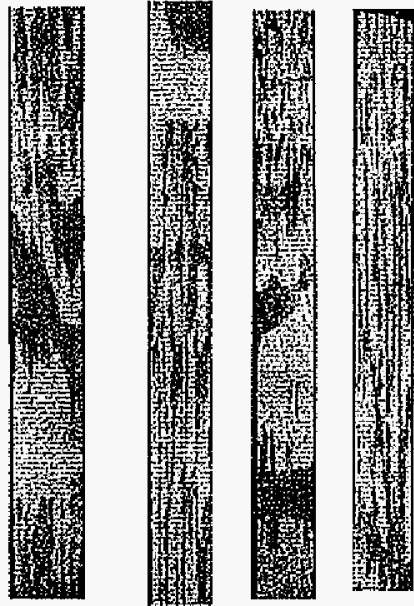

(b)

Fig. 11 Experimental observations of complex microstructure formation due to the dynamics of nucleation and growth processes. (a) Experimental conditions of composition and imposed velocity in the composite region, (b) Selected sections of experimental observations along the length of the sample. Significant dynamical effects are present that first produces bands, complex bands + composite structure and then the steadystate composition microstructure. 


\section{References}

1. H. W. Kerr and w. Kurz. Int. Mat. Rev., 41(4) (1996) 129-164.

2. W. J. Boettinger: Metall. Trans., 5(9) (1974) 2023-2031.

3.R. Trivedi, Metall. Mat. Trans. A, 26A(6) (1995) 1583-1590.

4. R. Trivedi and J. S. Park, J. Cryst. Growth, 235(I-4) (2002) 572-588.

5. J. S. Park and R. Trivedi, J. Cryst. Growth, 187(3-4) (1998) 511-515.

6. P. Mazumder, R. Trivedi and A. Karma, Metall. Mat. Trans. A, 31 A(4) (2000) 1233-1246.

7. P. Mazumder and R. Trivedi, Appl. Math. Modeling, 28 (2004) 109-125.

8. T. Lagrasso, B. C. Fuh and R. Trivedi, Metall. Mater. Trans. A, 36A(5) (2005) 1287-1300.

9. T.F. Bower, H.D. Brody, M.C. Flemings, Trans. Metall. AIME, $236(5)$ (1966) 624-634.

10. J. H. Lee and J. D. Verhoeven, J. Cryst. Growth, 144(3-4) (1994) 353-366.

11. H. Fredriksson (Ed.), Proceedings of the International Conference of Solidification and Casting, Sheffield, UK, Inst. of Metals, London, 1 (1977) 131-138.

12. M. Vandyoussefi, H. W.d Kerr and W. Kurz, Acta Mater., 48(9) (2000) 2297-2306.

13. S. Dobler, T. S. Lo, M. Plapp, A, Karma and W. Kurz, Acta mater., 52(9) (2004) $2795-$ 2808.

14. R. Trivedi, Scripta Mater., 53(1) (2005) 47-52.

15. T. S. Lo, A. Karma and M. Plapp, Phys. Rev. E, 63 (2001) 031504/1-15. 


\title{
CHAPTER 4: HETEROGENEOUS NUCLEATION IN PERITECTIC SYSTEMS
}

\author{
A paper to be submitted to Acta Materialia
}

J. H. Shin, J. H. Perepezko ${ }^{*}$, and R. Trivedi

\author{
Materials and Engineering Physics, Ames Laboratory (USDOE) and \\ Department of Materials Science and Engineering, \\ Iowa State University, Ames, IA 50011, USA \\ ${ }^{*}$ Department of Materials Science and Engineering, University of Wisconsin, Madison, Wi
}

\begin{abstract}
Directional solidification experiments have been carried out in the $\mathrm{Sn}-\mathrm{Cd}$ peritectic system to study the nucleation of the primary $\alpha$-phase and the peritectic $\beta$-phase. All experiments are done under high $\mathrm{G} / \mathrm{V}$ conditions $(\mathrm{G}$ and $\mathrm{V}$ being the thermal gradient and pulling velocity) where a non-steady-state, banded structure is present in which repeated bands of the primary phase and the peritectic phase form normal to the growth direction. The undercooling at which the primary and the peritectic phases nucleate is determined through the measurements of temperature and composition at the location where each band nucleates. Nucleation is observed to occur heterogeneously at the ampoule wall-liquid-solid triple junction. Since the contact angles at the wall play an important role in characterizing the nucleation undercooling, experiments have thus been carried out using three different ampoule materials, viz. quartz, carbon-coated quartz and tantalum. Nucleation undercooling
\end{abstract}


for each phase is found to be very small, from a fraction of a degree to about one degree. Relevant contact angles have been measured and it is shown that the very small measured nucleation undercooling values for each of these two phases are not consistent with the predictions of the classical nucleation theory. It is suggested that embryos with very few planes, of the order of the thickness of the interface, form at the wall-liquid interface with a very small non-equilibrium contact angle. These sub-critical clusters in the liquid near the wall, when they encounter the wall-liquid-solid triple junction, can become critical nuclei at some small undercooling.

\section{Introduction}

The evolution of two-phase microstructure during the solidification of alloys is generally complex, and it is governed by two critical criteria. (1) The relative nucleation undercooling of each phase may determine which phase would be dominant in the microstructure. As this phase grows, it is also possible that the nucleation undercooling of the second phase may be reached, so that both phases can be present. (2) Once both the phases are present, the microstructure is often governed by the relative growth rates of the two phases. These considerations are important not only for alloys in the two-phase region, but also for alloys in a single phase region under experimental conditions where stable and metastable phases can form $[1,2]$. Because of the complexities of the problem, most microstructure selection criteria are based on either the nucleation or the growth competition under steady-state conditions. It has been shown that directional solidification of peritectic alloys with composition in the two-phase region provides a way to examine quantitatively both the nucleation and the competitive growth processes [3, 4]. 
When an alloy in the two-phase region of a peritectic system is directionally solidified under high $\mathrm{G} / \mathrm{V}$ conditions, where $\mathrm{G}$ is the thermal gradient and $\mathrm{V}$ is the pulling velocity, a banded or an oscillatory microstructure forms in which repeated bands of the primary $\alpha$-phase and the peritectic $\beta$-phase form normal to the growth direction, as shown in Fig. 1 (a). In Fig. 1 (a), a complete band is shown in which the new phase, once nucleated at the wall, spreads laterally and stops the further growth of the parent phase. An oscillatory microstructure with partial band, shown in Fig. 1 (b), can also form in which a repeated nucleation of only $\beta$-phase occurs and it spreads slowly compared to the normal growth of the $\alpha$-phase $[4,8]$.

To form a complete band, repeated nucleation of the primary and the peritectic phase is required, whereas repeated nucleation of the peritectic phase is required for the partial band formation. Nucleation undercooling of the two phases also plays a critical role in the formation of the banded structure since they control the width of each phase, spacing between the bands, and the composition range over which banded microstructures can form [3]. The study of banded microstructures thus provides another valuable tool for quantitative measurements of nucleation undercooling.

Nucleation generally occurs heterogeneously, and the value of the nucleation undercooling depends on the preferred heterogeneous site. The nucleation of each phase can occur at: (a) the moving parent phase:liquid interface, which we shall refer to as the solidliquid interface or SL, (b) at the triple junction between the interface and the ampoule wall, WSL, (c) at the ampoule wall-liquid interface, WL, or (d) in the liquid, L, ahead of the interface, as shown in Fig. 2. Different nucleation undercooling will be required for these different nucleation sites, and they are governed by the critical free energy change, $\Delta \mathrm{G}^{*}$, at 
these sites, which depends on the critical radius and on the relevant contact angle values [2, $5]$.

The aim of this paper is to present experimental results in the Sn-Cd system on nucleation undercooling for the primary and the peritectic phase. Since nucleation depends on the contact angles at the wall, three different ampoule materials were used in this study. The advantages of this technique are: (i) the heterogeneous site at which nucleation occurs can be precisely characterized through metallographic observations, (ii) the nucleation undercooling can be measured through the interface temperature or the $\beta$-phase composition at the nucleation site, and (iii) a single nucleation event has been observed to occur in thin samples at the WSL site [4]. The feasibility of this technique has been demonstrated by Trivedi and Park [4] and Liu and Trivedi [6], who measured nucleation undercooling in Sn$\mathrm{Cd}$ and $\mathrm{Pb}-\mathrm{Bi}$ systems.

The results on nucleation undercooling are first analyzed by using the classical heterogeneous nucleation theory. Relevant contact angles were also measured in order to calculate the theoretical values of the nucleation undercooling at different heterogeneous sites. It is found that experimental values of undercooling are orders of magnitude smaller than those predicted theoretically by the classical model of heterogeneous nucleation at the WSL location. A new mechanism is proposed for the heterogeneous nucleation at the WSL junction.

\section{Experimental procedure}

Directional solidification experiments were carried out in the Sn-Cd system. This alloy system was selected since $\mathrm{Cd}$ is heavier than $\mathrm{Sn}$ so that fluid flow effects due to axial 
density difference will be suppressed in an upward Bridgman growth. Also, by using fine capillary samples, fluid flow due to any horizontal temperature gradient can be minimized. Earlier studies in this alloy system have demonstrated that diffusive growth is present in samples of diameter $<1.0 \mathrm{~mm}$ at very low velocities, of the order of $1.0 \mu \mathrm{m} / \mathrm{s}$, for the thermal profile of the apparatus used in this study $[4,7]$. In this study, all experiments were thus carried out in $0.4-0.6 \mathrm{~mm} \mathrm{I.D.} \mathrm{ampoules.} \mathrm{The} \mathrm{properties} \mathrm{of} \mathrm{the} \mathrm{Sn-Cd} \mathrm{system} \mathrm{are} \mathrm{listed} \mathrm{in}$ Table 1.

The technique of capillary sample assembly for directional solidification, described previously [4], was used. Since the nucleation at the ampoule-interface junction depends on the contact angles at the wall, three different ampoule materials (quartz, carbon-coated quartz and tantalum) were used in this study. We shall refer to the carbon-coated tube as carbon tube. In order to obtain a carbon-coated tube, acetone is inserted into a $0.6 \mathrm{~mm}$ quartz tube by capillary force and coated uniformly on the inside wall. The quartz tube was then heated to about $1100^{\circ} \mathrm{C}$, where acetone decomposed into carbon and water. This processing left a coating of carbon on the inside wall of the quartz tube.

Experiments were carried out in Sn-0.9 wt\% Cd alloy, with thermal gradient of 19.5 $\mathrm{K} / \mathrm{mm}$ and growth velocity ranging from 0.7 to $2.8 \mu \mathrm{m} / \mathrm{s}$. For these conditions, a stable planar morphology is predicted for velocities below $2.8 \mu \mathrm{m} / \mathrm{s}$. Initially, the alloy was melted in a larger $4.0 \mathrm{~mm}$ ampoule, and a capillary sample was inserted at the center of the larger quartz ampoule. The alloy was rapidly grown to a distance of $20.0 \mathrm{~mm}$ and held stationary in order to stabilize the thermal profile. The alloy was then solidified at a fixed pulling rate over a distance of about $70 \mathrm{~mm}$, after which it was quenched by rapidly moving the sample in the liquid metal cooling region. Microstructure was observed with OM (Optical Microscope) and 


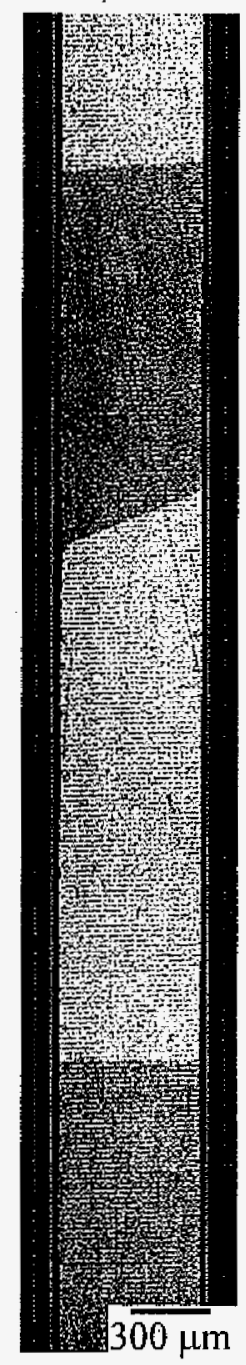

(a)

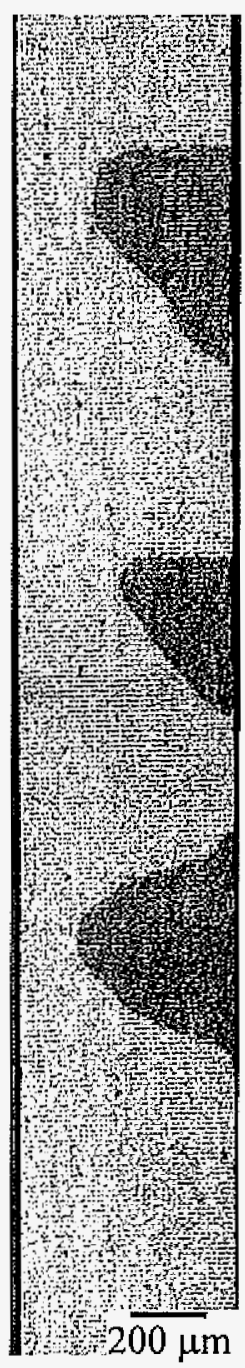

(b)

Fig. 1 (a) A complete band structure in $0.9 \mathrm{wt} \% \mathrm{Cd}$ for $\mathrm{G} / \mathrm{V}=6964 \mathrm{~K} \cdot \mathrm{s} / \mathrm{mm}^{2}$. (b) Partial band structure in $\mathrm{Sn}-0.8$ wt $\% \mathrm{Cd}$. 


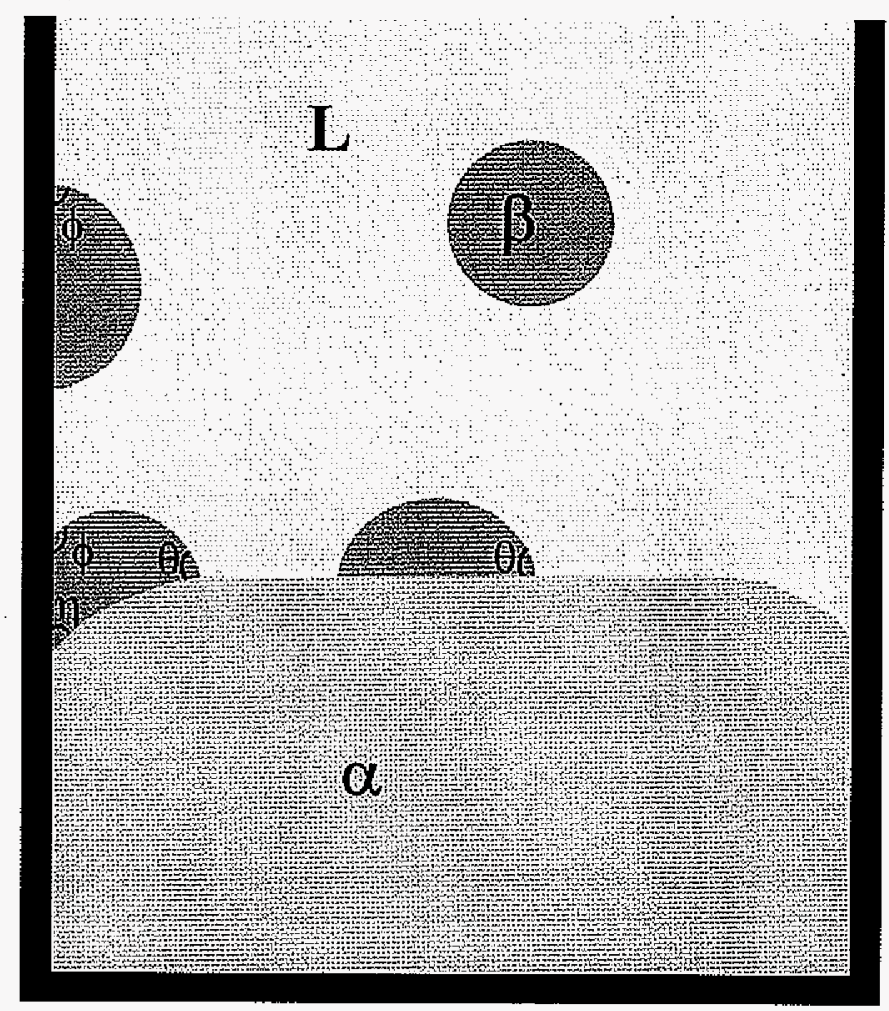

Fig. 2 Different possible heterogeneous sites for the nucleation of the $\beta$-phase at or ahead of the $\alpha$-liquid interface. The contact angles at the wall-liquid $(\phi), \alpha$-liquid $(\theta)$ and the wall-interface junction ( $\eta$ ) are shown. 
Table 1 Physical properties of Sn-Cd alloy

\begin{tabular}{llll}
\hline Property & Symbol & Value & Unit \\
\hline Peritectic temperature & $\mathrm{T}_{p}$ & 496 & $\mathrm{~K}$ \\
Diffusion coefficient & $D_{l}$ & $1.6 \times 10^{-3}$ & $\mathrm{~mm}^{2} / \mathrm{s}$ \\
Distribution coefficient & $k^{\alpha}$ & 0.163 & \\
& $k^{\beta}$ & 0.248 & $\mathrm{~K} / \mathrm{wt} \%$ \\
Liquidus slope & $m^{\alpha}$ & -2.368 & $\mathrm{~K} / \mathrm{wt} \%$ \\
& $m^{\beta}$ & -0.735 & $\mathrm{~J} / \mathrm{m}^{2}$ \\
S/L interface energy & $\gamma^{\alpha}$ & 0.074 & $\mathrm{~J} / \mathrm{m}^{2}$ \\
& $\gamma^{\beta}$ & 0.0603 & $\mathrm{~J} / \mathrm{m}^{3} \mathrm{~K}$ \\
Entropy of fusion & $\Delta \mathrm{S}^{\alpha}$ & 780000 & $\mathrm{~J} / \mathrm{m}^{3} \mathrm{~K}$ \\
& $\Delta \mathrm{S}^{\beta}$ & 561600 & $\mathrm{~m} \cdot \mathrm{K}$ \\
Gibbs-Thomson Coefficient & $\Gamma_{\alpha}$ & $0.948 \times 10^{-7}$ & $\mathrm{~m} \cdot \mathrm{K}$ \\
& $\Gamma_{\beta}$ & $1.074 \times 10^{-7}$ & $\mathrm{~m}$ \\
Atomic volume & $\mathrm{v}^{\prime \alpha}$ & $0.054 \times 10^{-27}$ & $\mathrm{~m}$ \\
\hline \hline
\end{tabular}


SEM (Scanning Electron Microscope) after etching with a $4 \%$ solution of nitric acid in lactic acid.

For an independent assessment of the effect of ampoule material on nucleation temperature, few experiments were also carried out with the capillary assembly containing

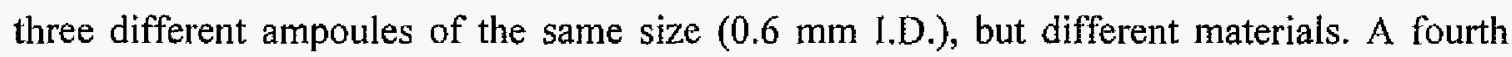
capillary tube that contained a calibrated thermocouple was also placed at the center to obtain the temperature profile. All four tubes were placed in a larger tube of $4.0 \mathrm{~mm}$ diameter, and they were first equilibrated such that the initial solid-liquid interface position in all three samples was at the same or the initial interface temperature was the same in all samples. The relative distances where the $\beta$-phase nucleated were measured to calculate the relative nucleation undercooling in the three ampoule materials.

To obtain the nucleation undercooling for the $\alpha$ - and the $\beta$-phases, temperatures where the $\alpha$-phase and the $\beta$-phase nucleate were determined from the thermal profile obtained from the thermocouple that was placed in the outer ampoule. In addition, compositions were also measured in the $\beta$-phase at the $\alpha: \beta$ and the $\beta: \alpha$ interfaces with WDS, and these compositions were related to the nucleation temperature by using the phase diagram. Contact angles at the ampoule wall: $\alpha$ :liquid were measured in Sn-0.9 wt\% Cd alloys that were grown by $3.0 \mathrm{~mm}$ at the growth rate of $2.5 \mu \mathrm{m} / \mathrm{s}$ and quenched rapidly before the peritectic phase nucleated. Contact angles at the ampoule wall: $\beta$ :liquid location were measured in another rapidly quenched sample after the first $\beta$-phase nucleation occurred. Contact angles were measured using SEM images after successively polishing the sample longitudinally and examining the appropriate section to minimize the sectioning effect. 


\section{Results}

\subsection{Nucleation undercooling}

Since the location at which nucleation occurs can not be precisely determined from one cross-section, detailed successive sectioning technique was used to examine the threedimensional shape of the complete and partial bands, as shown in Fig. 3. Generally, the Iocation of nucleation was found to be close to the central section of the sample. The temperature at the location of the nucleation was then obtained from the thermal profile. Also, the $\beta$-phase compositions were measured at the $\alpha: \beta$ and $\beta: \alpha$ interfaces, and they were converted to the $\alpha$-phase and the $\beta$-phase nucleation temperature, $\mathrm{T}_{N}{ }^{\alpha}$ and $\mathrm{T}_{\mathrm{N}}{ }^{\beta}$, by using the phase diagram, as shown by filled circles in Fig. 4. Note that the undercooling values for both the phases were obtained from the composition measurements in the $\beta$-phase only since the composition in the $\alpha$-phase can not be measured accurately since it decomposes upon cooling. From the nucleation temperatures, nucleation undercooling values were obtained by using the procedure described earlier $[4,6]$.

Nucleation undercooling for the $\alpha$-phase and the $\beta$-phase in carbon ampoule were measured for several bands in two different samples to examine the experimental variation in the nucleation undercooling values, and the results are shown in Fig. 5. The variation is very small. These nucleation undercooling values were measured in experiments in which only a single capillary sample was used. To examine the effect of ampoule material on nucleation undercooling, experiments were carried out in which ampoules of three different materials were placed in a large ampoule along with a fourth tube containing the thermocouple. The sample was first equilibrated so that the initial interface positions in all the ampoules were at 
the same location, as shown in Fig. 6. The locations of the nucleation of the $\beta$-phase in these three ampoules are marked in Fig. 6, and the absolute and relative temperature at the $\beta$-phase nucleation locations in the three ampoules were determined. The measured undercooling values are shown in Fig. 7 for the three ampoules. The undercooling values from temperature measurements were found to agree with those from the composition measurements. All the results on the nucleation undercooling are given in Table 2 . The nucleation undercooling for the tantalum ampoule was found to be the smallest and that for the quartz was the largest, indicating the effect of different contact angles at the ampoule wall. Note that once the $\beta$ phase is nucleated, it grows along the ampoule wall in the Ta tube, forms partial bands in the carbon tube, and shows complete band in the quartz ampoule.

\subsection{Contact angles}

To compare the experimental results with the nucleation model, three contact angles, $(\theta, \phi, \eta)$ are required for each phase in a given ampoule material, as shown in Fig. 8. Thus, a total of 6 angles are required for each ampoule, and a total of 14 contact angles are required for the three ampoules since the angle $\theta$ is the same for all ampoules. However, not all contact angles are independent, and the contact angles for the $\alpha$-phase are related to the corresponding contact angles for the $\beta$-phase, as shown in Appendix 1 , so that the knowledge of only three contact angles for each ampoule material is required. Four contact angles were measured experimentally in several quenched samples by taking into account the threedimensional shape of the interface, as shown in Fig. 3. Several contact angle measurements were carried out for each angle, and the optimum values were selected that satisfied the 


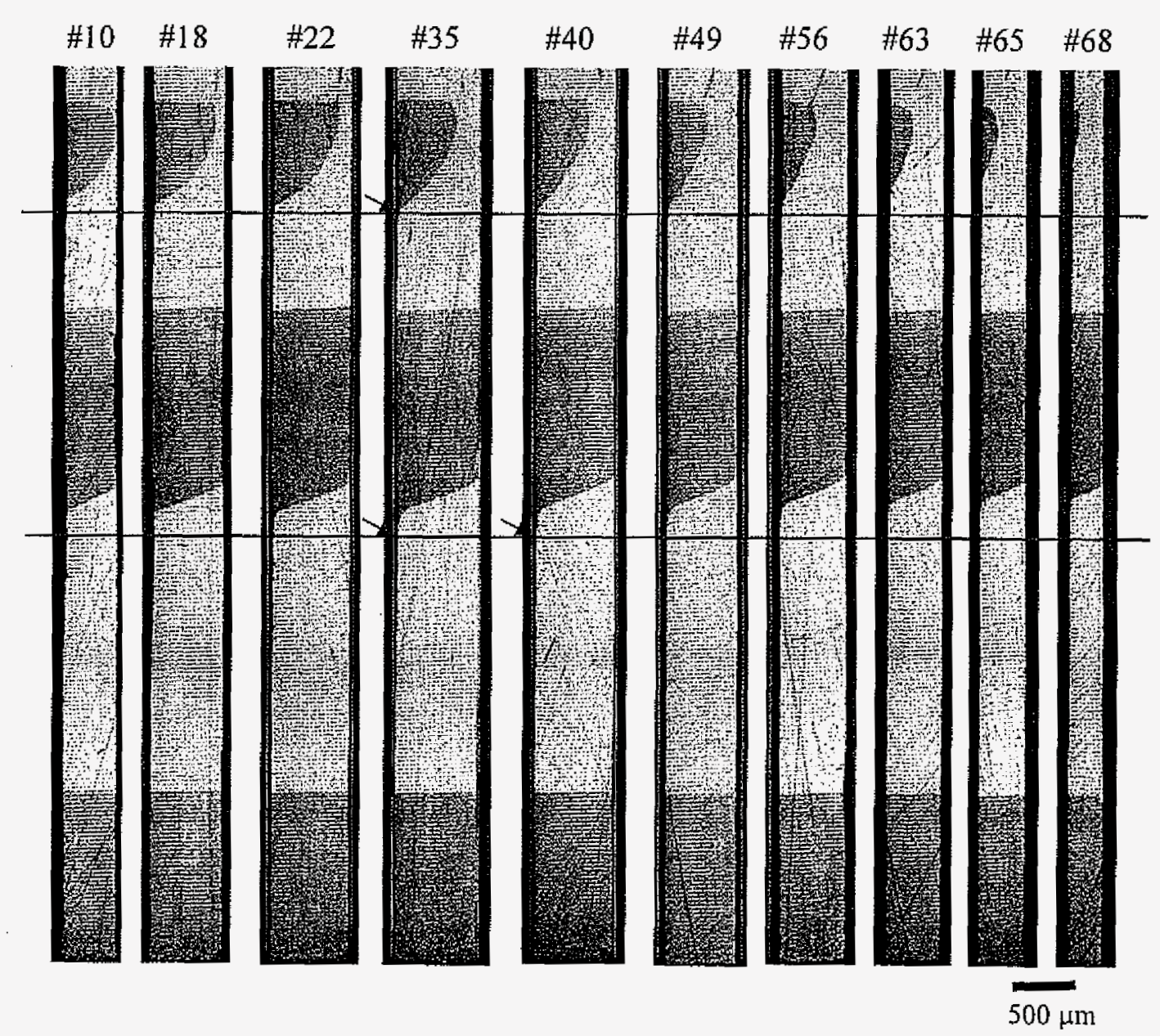

Fig. 3 Successive cross-sections to locate the nucleation site in three-dimensions. 


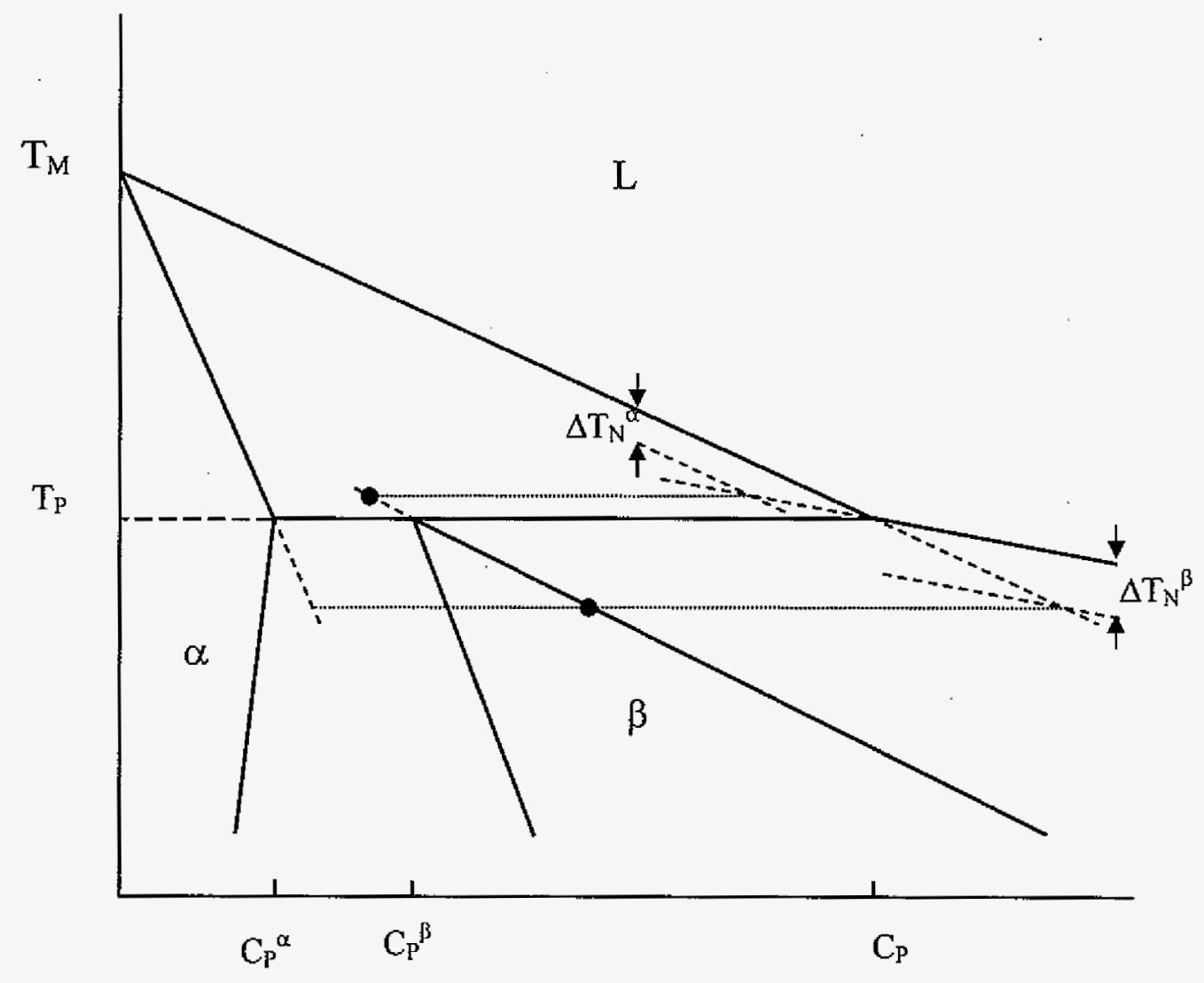

Composition

Fig. 4 Phase diagram showing nucleation undercooling from the composition in the $\beta$-phase at the nucleus location. 


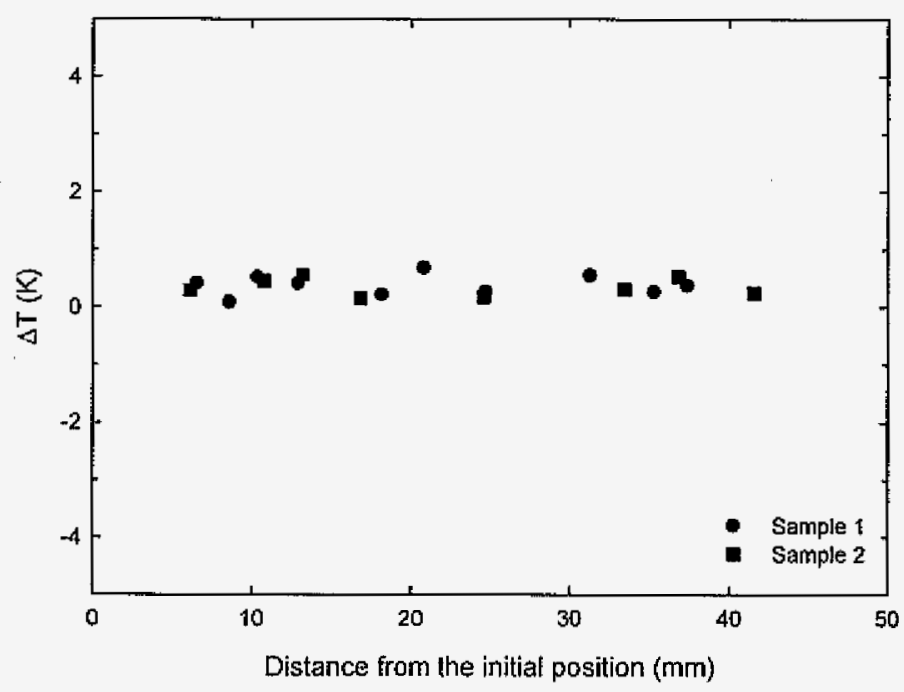

(a)

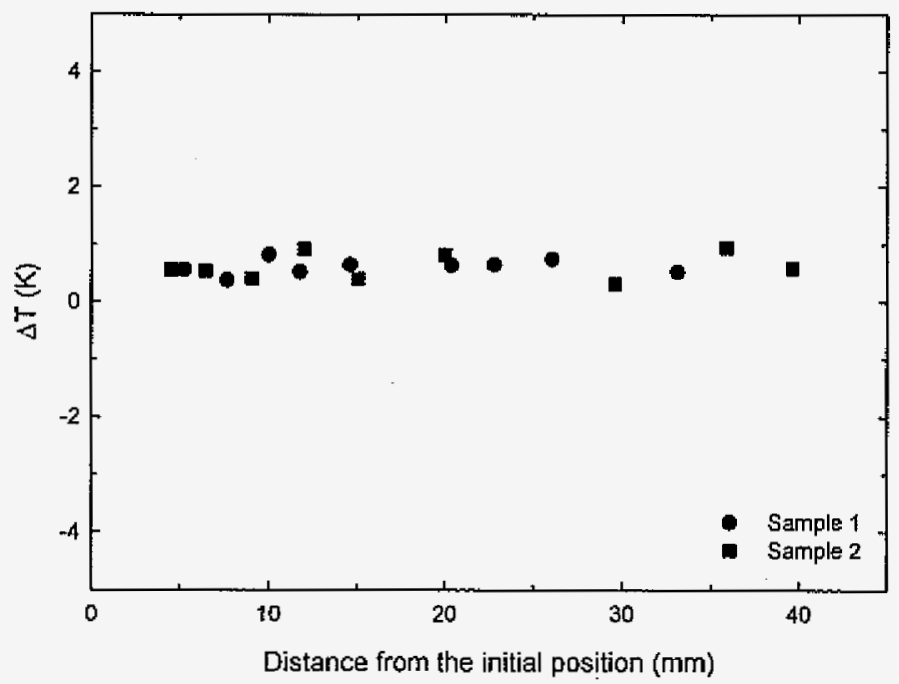

(b)

Fig. 5 Nucleation undercooling for (a) the $\alpha$-phase, and (b) the $\beta$-phase in carbon ampoules, measured for several bands in two different samples. 


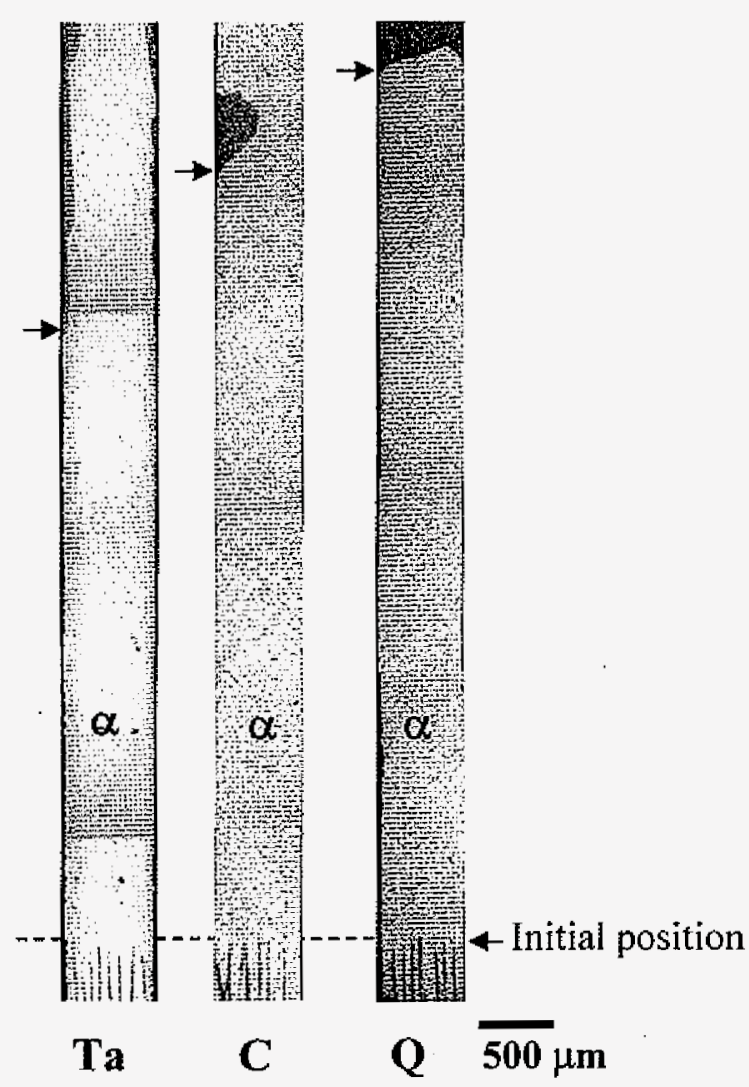

Fig. 6 The initial interface location and the location of the $\beta$-phase nucleation in Ta, carbon and quartz ampoules for the experiment in which all the three ampoules were placed in a larger quartz ampoule and solidified in a given experiment. 


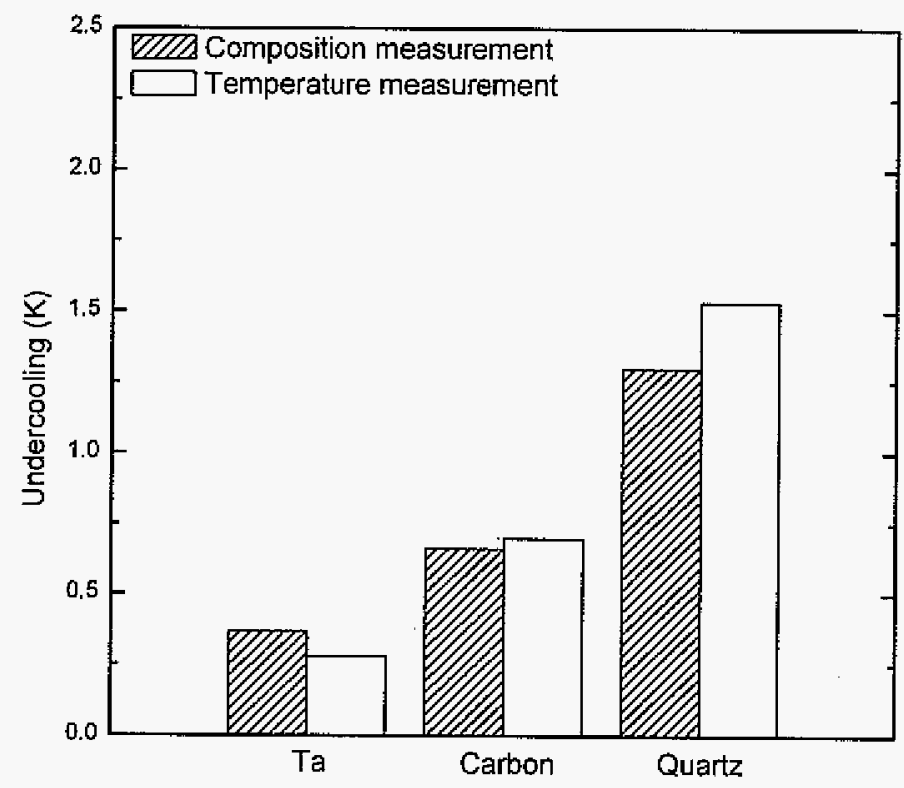

Fig. 7 Nucleation undercooling for the $\beta$-phase in tantalum, carbon and quartz ampoules, determined by temperature as well as composition measurements. 

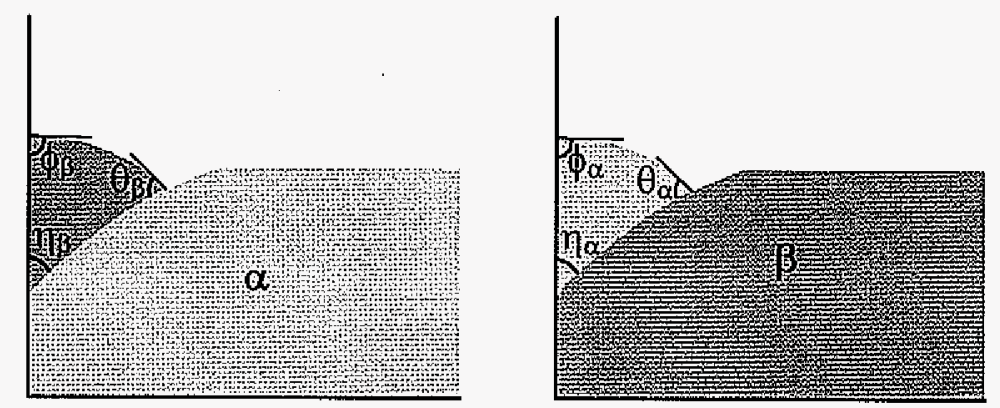

Fig. 8 Relevant contact angles for the nucleation of the $\alpha$-phase and the $\beta$-phase at the wallinterface triple junction. 
Table 2. Uncleation undercooling with tube materials.

\begin{tabular}{llll}
\hline \hline & Ta & $\mathrm{C}$ & $\mathrm{Q}$ \\
\hline$\alpha$-phase & $0.18(\mathrm{~K})$ & $0.2(\mathrm{~K})$ & $0.2(\mathrm{~K})$ \\
$\beta$-phase & $0.38(\mathrm{~K})$ & $0.66(\mathrm{~K})$ & $1.3(\mathrm{~K})$ \\
\hline
\end{tabular}

Table 3 Contact angle at the carbon tube and the tantalum tube.

\begin{tabular}{llrlll}
\hline & $\mathrm{C}$ & $\mathrm{Ta}$ & & $\mathrm{C}$ & $\mathrm{Ta}$ \\
\hline$\phi_{\alpha}$ & $106^{\circ}$ & $136^{\circ}$ & $\phi_{\beta}$ & $100^{\circ}$ & $105^{\circ}$ \\
$\theta_{\alpha}$ & $100.67^{\circ}$ & $99^{\circ}$ & $\theta_{\beta}$ & $90^{\circ}$ & $90^{\circ}$ \\
$\eta_{\alpha(\#)}$ & $97.71^{\circ}$ & $120^{\circ}$ & $\eta_{\beta(*)}$ & $82.29^{\circ}$ & $60^{\circ}$ \\
\hline \hline
\end{tabular}

(\#) Calcvulated 
constraints given in Appendix 1. The values of the contact angles are listed in Table 3.

\section{Discussion}

The nucleation undercooling values will be first compared with the predictions of the classical heterogeneous nucleation theory for the heterogeneous nucleation at the WSL triple junction. It will be shown that the theoretically predicted nucleation undercooling values are over two orders of magnitude larger than the experimentally observed values. A new plausible model of nucleation will then be proposed in which clusters of atoms present at the liquid-wall interface, when they encounter the interface, coalesce with the embryos at the wall-interface junction to form a critical size nucleus.

\subsection{Nucleation at the WSL junction}

Experimental results show that nucleation of the primary and the peritectic phases occurs at the wall-interface junction. The preferred heterogeneous nucleation site is governed by the free energy of formation of the critical nucleus, $\Delta G^{*}$, which depends on the values of the relevant contact angles. For heterogeneous nucleation at the WSL triple junction, the critical free energy of nucleation is given by:

$$
\Delta G^{*}=\left[\frac{16 \pi \gamma^{3}}{3 \Delta S_{v}^{2} \Delta T_{N}^{2}}\right] \mathrm{F}(\theta, \phi, \eta)
$$

in which $\gamma$ is the solid-liquid interface energy, $\Delta S_{\mathrm{v}}$ is the entropy change per unit volume and $\Delta T_{N}$ is the undercooling at which nucleation occurs. The function $F(\theta, \phi, \eta)$ has been derived by Trivedi [5] as 


$$
\begin{aligned}
F(\theta, \phi, \eta)= & \frac{1}{4 \pi}\left[\sin ^{2} \theta \cos \theta \cos ^{-1}\left(\cot \theta \cot \left(\eta_{1} / 2\right)\right)\right. \\
& \left.+\sin ^{2} \phi \cos \phi \cos ^{-1}\left(\cot \phi \cot \left(\eta_{2} / 2\right)\right)\right] \\
& +\frac{1}{4 \pi}\left[\cos ^{2} \theta \cot \left(\eta_{1} / 2\right)\left(\sqrt{\sin ^{2} \theta-\cos ^{2} \theta \cot ^{2}\left(\eta_{1} / 2\right)}\right)\right. \\
& \left.+\cos ^{2} \phi \cot \left(\eta_{2} / 2\right)\left(\sqrt{\sin ^{2} \phi-\cos ^{2} \phi \cot ^{2}\left(\eta_{2} / 2\right)}\right)\right] \\
& -\frac{1}{2 \pi}\left[\cos \theta \cos ^{-1}\left[\cot \theta \cot \left(\eta_{1} / 2\right)\right]+\cos \phi \cos ^{-1}\left[\cot \phi \cot \left(\eta_{2} / 2\right)\right]\right] \\
& +\frac{1}{8 \pi}\left[\cos \left(\frac{\cos \left(\eta_{1} / 2\right)}{\sin \theta}\right)+\cos ^{-1}\left(\frac{\cos \left(\eta_{2} / 2\right)}{\sin \phi}\right)\right]
\end{aligned}
$$

where $\cot \left(\eta_{1} / 2\right)=(\cos \phi+\cos \theta \cos \eta) / \cos \theta \sin \eta$, and $\cot \left(\eta_{2} / 2\right)=(\cos \theta+\cos \phi \cos \eta) / \cos \phi \sin \eta$. The nucleation at the wall-interface junction can occur only when the quantity under the square root sign is positive. It can be shown that this requires the following four conditions to be satisfied: $\theta+\eta+\phi>\pi, \theta-\eta+\phi<\pi, \theta+\eta-\phi<\pi$, and $\eta+\phi-\theta<\pi$. These conditions with an equality sign are equations of planes in the $\theta, \phi, \eta$ coordinates, and if we consider a cube with length $\pi$, then they are equivalent to four $<111>$ planes in cubic crystals. Thus nucleation at the WSL junction will be possible only when the point representing the three contact angles lies within the central tetrahedron shown in Fig: 9.

There are also four other smaller tetrahedrons in the cube, shown in Fig. 9 with an apex at points $A, B, C$, and $D$ in which nucleation at the wall-interface junction will not occur. As shown by Trivedi [5], they represent conditions for nucleation at the solid-liquid interface away from the wall, at the wall-liquid interface, and in the liquid. Since nucleation was found to occur only at the wall-interface junction in all our experiments, the points representing the three angles in different ampoule materials should lie within the central tetrahedron. Since the contact angle $\theta$ does not depend on the ampoule material, we examine the cross-section 


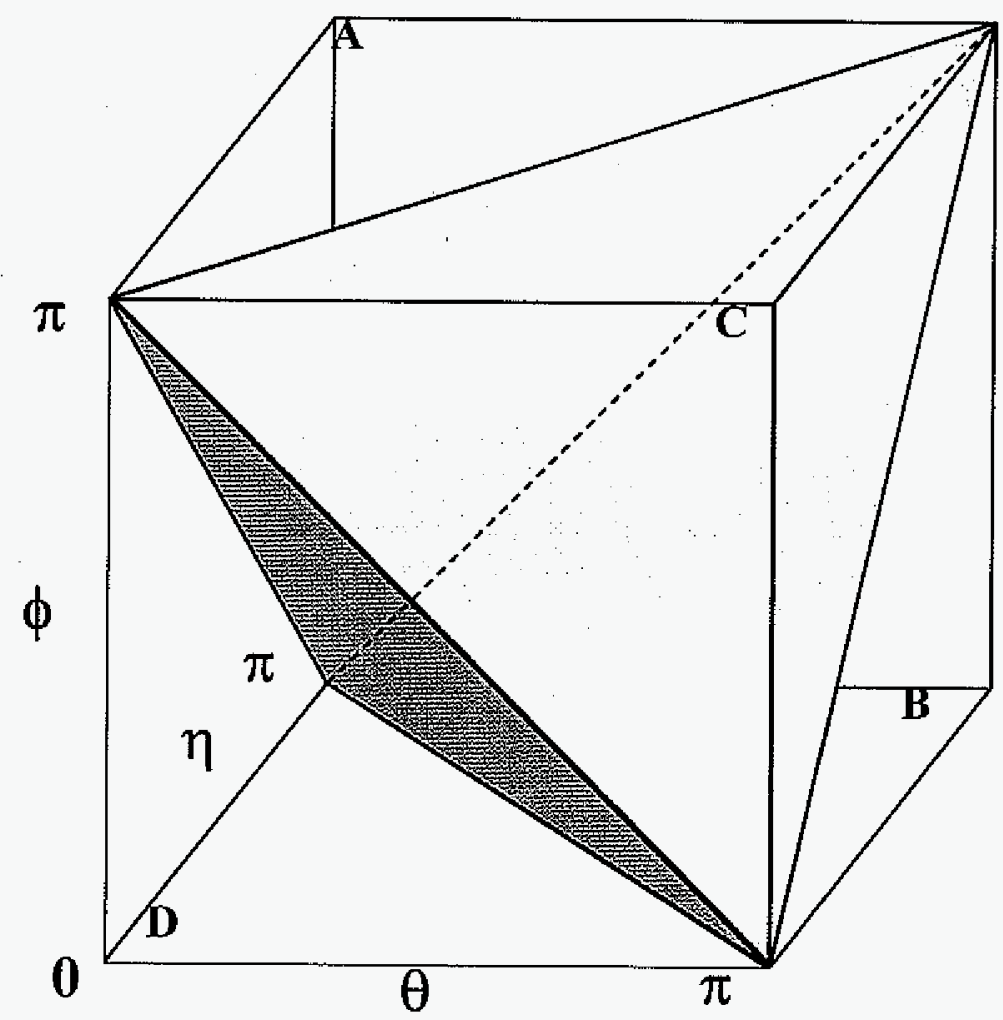

Fig. 9 Nucleation map showing the regimes of different preferred heterogeneous nucleation sites depending on the values of the contact angles $\theta, \phi, \eta$. The central tetrahedron corresponds to the nucleation site of the wall-liquid-solid triple junction. The tetrahedrons with apex at $A$ and $B$ represent the nucleation sites of the solid-liquid interface and the wall-liquid interface, respectively. The tetrahedrons with apex at $C$ and $D$ show regions for which no nucleation on any substrate is possible, and nucleation can occur heterogeneously in the bulk liquid only. 


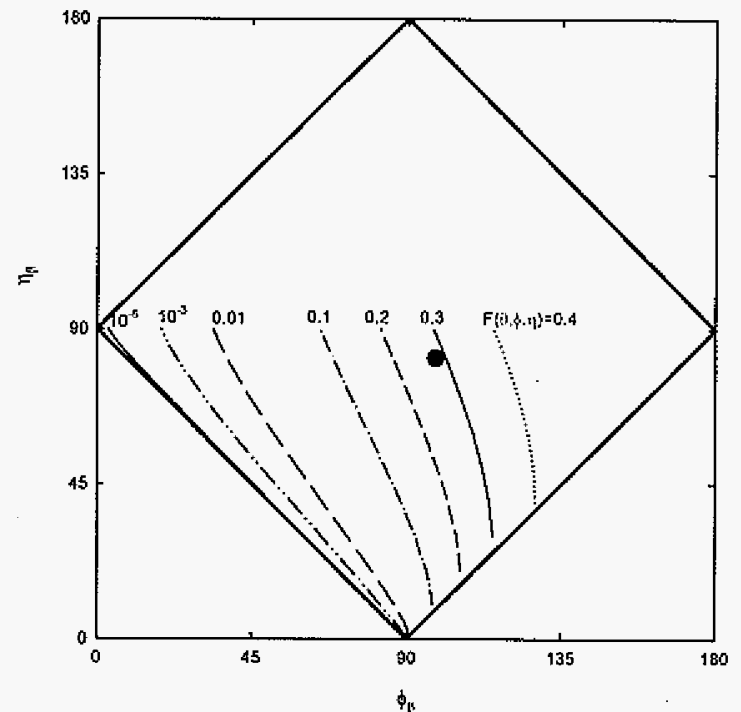

(a)

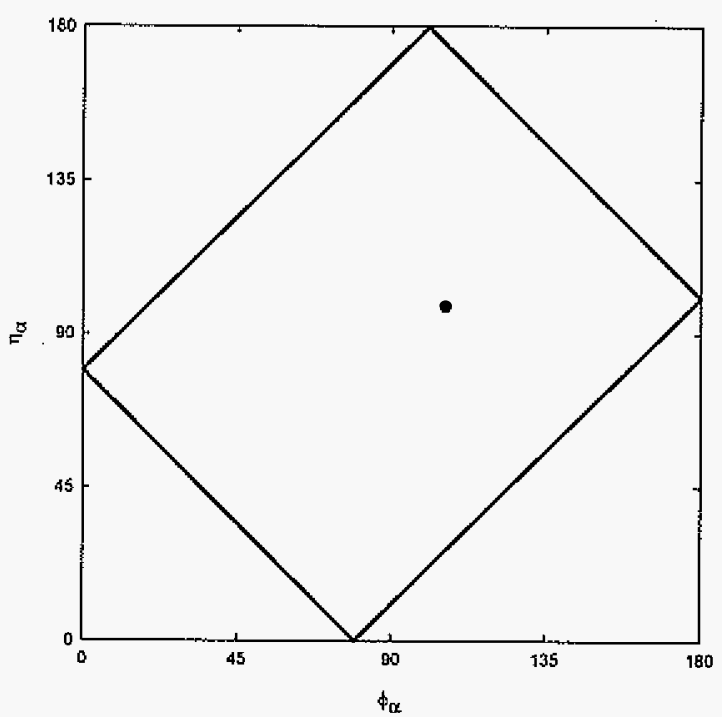

(b)

Fig. 10 Cross-sections of the nucleation map for: (a) the nucleation of the $\beta$-phase for $\theta_{\beta}=$ $90^{\circ}$, and (b) the nucleation of the $\alpha$-phase for $\theta_{\alpha}=100.67^{\circ}$. The experimental values of the contact angles fall within the central tetrahedron. 
of the central tetrahedron for $\theta_{\beta}=90^{\circ}$ and $\theta_{\alpha}=100.67^{\circ}$, as shown in Figs. 10 (a) and 10 (b), respectively. The experimental values of $\phi$ and $\eta$ for the nucleation of the $\alpha$-phase and the $\beta$ phase are shown in the Fig. 10 for the carbon ampoule. Note that both these points fall within the central tetrahedron, which is necessary for the nucleation at the wall-interface junction. However, the values of the functions $F_{\alpha}(\theta, \phi, \eta)$ and $F_{\beta}(\theta, \phi, \eta)$ for the measured contact angles are 0.46 and 0.294 , respectively, which are orders of magnitude larger than those required by the classical nucleation model, as we shall now discuss.

\subsection{Nucleation undercooling}

Experimental results on the undercooling for the nucleation of the $\alpha$-phase and the $\beta$ phase in carbon tubes can now be tested against the classical nucleation theory in which the nucleation process occurs through thermal fluctuations to form a critical nucleus. The nucleation rate, $I$, on a substrate is given by the relationship [1]:

$$
\mathrm{I}=\left[\frac{6 D}{\lambda^{2}}\right]\left[\frac{4 \pi \mathrm{r}^{* 2} f_{s}}{\lambda^{2}}\right] \mathrm{ZC}_{\mathrm{w}} \mathrm{e}^{-\left(\Delta \mathrm{G}^{*} / k \mathrm{~T}\right)}
$$

Where $D$ is the diffusion coefficient in the liquid, $Z$ the $Z$ eldovich non-equilibrium factor, $\mathrm{C}_{\mathrm{w}}$ the number sites available in the ampoule for nucleation at the wall-interface junction, $\lambda$ the distance between the atoms, and $f_{\mathrm{s}}$ is the fraction of atoms on the surface of the nuclei compared to the number of surface atoms for a spherical nucleus. The nucleation undercooling is then obtained when $I=1$, which can be written as.

$$
\Delta \mathrm{G}^{*}=k \mathrm{~T} \ln \left[\frac{24 D Z \mathrm{C}_{\mathrm{w}}}{\lambda^{4}} 4 \pi \mathrm{r}^{* 2} f_{\mathrm{s}}\right]=\mathrm{A} k \mathrm{~T}
$$

where $A$ is given by [1]: 


$$
A=\ln \left[\frac{24 \pi r^{* 2} f_{s} D}{\lambda^{4}} Z C_{w}\right]
$$

Substituting the value of $\Delta \mathrm{G}^{*}$ from equation (1), we obtain the nucleation undercooling at the wall-interface junction as

$$
\Delta T_{N}=\left(\frac{16 \pi \gamma^{3}}{3 \mathrm{~A} \Delta \mathrm{S}_{\mathrm{v}}^{2} k T_{N}}\right)^{\mathrm{l} / 2}[\mathrm{~F}(\theta, \phi, \eta)]^{1 / 2}
$$

Since $A$ is a logarithmic term, it is not very sensitive to the values of the parameters so that its value for the nucleation at the wall-interface junction can be estimated by using the typical values of the parameters, $C_{w}=10^{20}$, and $Z=10^{-2}$, and by taking the values for the $\beta$-phase in liquid in the Sn-Cd system: $D_{1}=1.6 \times 10^{-9} \mathrm{~m}^{2} / \mathrm{s}, \lambda=2.96 \times 10^{-10} \mathrm{~m}$, and $f_{\mathrm{s}}=0.25$. The value of $\mathrm{r}^{*}$ depends on nucleation undercooling. We first consider experimental value of the undercooling to calculate the critical radius, and then reiterate to obtain the $r^{*}$ value at the calculated nucleation undercooling. The value of $A=74$ is obtained for heterogeneous nucleation at the wall-interface junction.

The nucleation undercooling for the $\beta$-phase and the $\alpha$-phase is thus obtained as:

$$
\Delta T_{N}^{\beta}=\left(\frac{\pi \gamma_{\beta l}^{3}}{14 \Delta S_{v \beta}^{2} k T_{N}^{\beta}}\right)^{1 / 2}\left[F_{\beta}(\theta, \phi, \eta)\right]^{1 / 2}
$$

and

$$
\Delta T_{N}^{\alpha}=\left(\frac{\pi \gamma_{c l}^{3}}{14 \Delta \mathrm{S}_{\mathrm{v} \alpha}^{2} \mathrm{kT}_{\mathrm{N}}^{\alpha}}\right)^{1 / 2}\left[\mathrm{~F}_{\alpha}(\theta, \phi, \eta)\right]^{1 / 2}
$$

The theoretical values of the undercooling can now be obtained by using the measured contact angle values for the carbon-coated ampoule, which give $F_{\alpha}(\theta, \phi, \eta)=0.46$ 
and $F_{\beta}(\theta, \phi, \eta)=0.294$ that predicts nucleation undercooling of the $\alpha$-phase and the $\beta$-phase as $\Delta \mathrm{T}_{\mathrm{N}}{ }^{\alpha}=113 \mathrm{~K}$ and $\Delta \mathrm{T}_{\mathrm{N}}{ }^{\beta}=96.64 \mathrm{~K}$, respectively. These values are more than two orders of magnitude larger than the experimentally observed value of $0.20 \mathrm{~K}$ and $0.66 \mathrm{~K}$, respectively.

We may consider the discrepancy due to the errors in experimental measurements of nucleation undercooling or contact angles. Several nucleation undercooling values were measured in two different samples, as shown in Fig. 5, and they all give results within a narrow band of undercooling. In addition, the same technique was used by Trivedi and Park [4] and by Liu [9] to measure nucleation undercooling values for the $\alpha$-phase and the $\beta$-phase in the Sn-Cd systern, and they obtained values close to those found in this study. In addition, the nucleation undercooling in different ampoules was also of the same order of magnitude, so that the very large discrepancy can not be attributed to the error in the measurements of nucleation undercooling.

Since the precise measurements of the contact angles are difficult, we now examine if the discrepancy could be due to the error in the contact angle values. In fact, a more reliable conclusion can be made by first assuming that the classical nucleation model is operative, and then determine the contact angles required to obtain the experimentally measured nucleation undercooling values. As shown in Appendix 1, only one contact angle needs to be known accurately, the other five angles could be obtained by using equations (A3) to (A7) if nucleation undercooling follows the classical nucleation model. We consider the measured angle $\theta_{\beta}=90^{\circ}$ as an independent angle. By substituting the values of the nucleation undercooling in equations (7a) and (7b), one obtains:

$$
\mathrm{F}_{\beta}(\theta, \phi, \eta)=1.89 \times 10^{-5}
$$

and 


$$
F_{\alpha}(\theta, \phi, \eta)=1.83 \times 10^{-6}
$$

These values are very small, and we may examine the contact angles required to give these small values. We first use equation (2) to calculate pair of angles $(\phi, \eta)$ that will give a constant value of $\mathrm{F}(\theta, \phi, \eta)$. The contact angles $\phi$ and $\eta$ for the $\beta$-phase, required to give $F_{\beta}(\theta, \phi, \eta)=1.89 \times 10^{-5}$, are shown in Fig. 11 (a), and these pair of angles should be very close to the boundary of the nucleation map for nucleation at the WSL site. For the nucleation of the $\alpha$-phase, we construct a similar nucleation map for $\theta_{\alpha}=100.9^{\circ}$. The values of the $(\theta, \eta)$ required to give $F_{\alpha}(\theta, \phi, \eta)=1.83 \times 10^{-6}$, as shown in Fig. $11(\mathrm{~b})$. This line is also very close to the limit of stability of the nuclei at the WSL junction. Thus, classical nucleation model can explain the observed results only if the contact angles for the two phases lie on the respective lines close to their limit of stability. However, we have shown that the contact angles for the two phases are not independent in that if the contact angles for the $\beta$-phase are known, then the contact angles for the $\alpha$-phase are fixed by equations (A3) to (A5). If we consider that the contact angles for the $\beta$-phase fall on the required line in Fig. 11 (a), then the corresponding contact angels for the $\alpha$-phase are fixed and shown in Fig. 11 (b) as points, and they are significantly different from the line that will give $F_{\alpha}(\theta, \phi, \eta)=1.83 \times 10^{-6}$. Thus, no contact angle values are found that can satisfy both the required relationships given by equations (8a) and (8b) simultaneously. It is thus clear that experimentally observed nucleation undercooling can not be obtained from the classical nucleation theory at a given heterogeneous site. We shall now propose a new nucleation mechanism to explain the experimental results. 


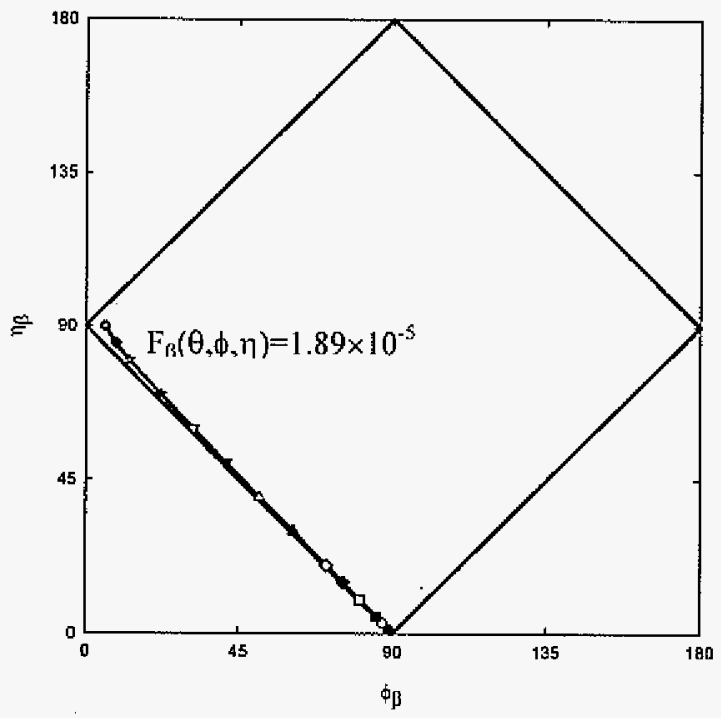

(a)

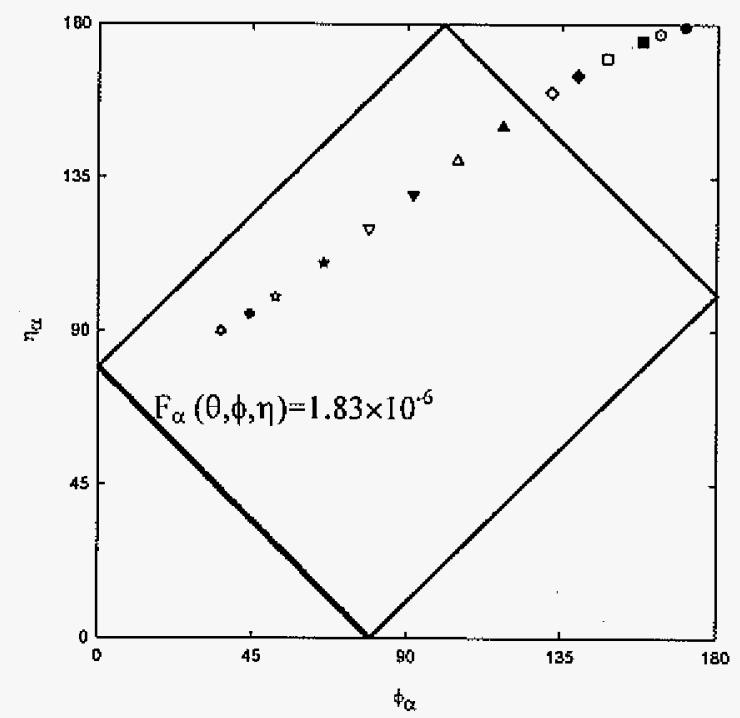

(b)

Fig. 11 The required contact angles for nucleation of (a) the $\beta$-phase or (b) the $\alpha$-phase according to the classical nucleation theory, which show the required angles to be close to the limit of stability. The points in Fig. (b) shows the contact angles in the $\alpha$-phase that would be present if the contact angles for the nucleation of the $\beta$-phase are along the line in Fig. (a). 


\subsection{Nucleation mechanism}

We consider the basic process of nucleation in terms of thermally activated cluster formation that can lead to the critical size nuclei at some definite undercooling. In the experiments presented here the nucleation undercooling is very small, where the cluster size will be significantly smaller than the critical cluster size if the clustering process occurs at the wall-interface triple junction. A new mechanism that may operates to form a critical cluster at some small but fixed undercooling will now be examined. It is proposed that cluster formation occurs at different heterogeneous sites in the undercooled liquid. We first consider cluster formation near a wall in the liquid ahead of the solid-liquid interface. These clusters are much smaller than the critical cluster size required for the nucleation of solid at the wallliquid interface. However, when these embryos encounter the solid-liquid interface, they could become critical clusters at some undercooling since the critical cluster size is significantly smaller for nucleation at the WSL triple junction. Thus, nucleation can occur when embryos at one heterogeneous site become critical cluster when it encounters another heterogeneous site as shown in Fig. 12. In reality, cluster formation occurs at both the heterogeneous sites, i.e. at the liquid-wall and at the wall-interface junction, so that the cluster at the wall-liquid, when it encounters the solid-liquid interface, will coalesce with the cluster at the wall-interface junction. Nucleation occurs at an undercooling where the total number of atoms in both the clusters is equal to the critical number of atoms required for nucleation at the wall-interface junction.

To illustrate this concept, we first consider the cluster formation at the WL and the WSL sites. The free energy change for cluster formation at the WSL site as a function of number of atoms, $n$, in the cluster is given by: 


$$
\Delta G(n)=\mathrm{n}_{\mathrm{n}} \Delta \mathrm{G}_{\mathrm{v}}+4 \pi\left(\frac{3 \mathrm{n} \mathrm{v}_{\mathrm{s}}}{4 \pi}\right)^{2 / 3} \gamma_{s l}(\mathrm{~F}(\theta, \phi, \eta))^{1 / 3}
$$

The free energy change for cluster formation at the WL site for the same number of atoms in a cluster will be given by the above equation with $F(\phi)$ replacing $F(\theta, \phi, \eta)$. It is more appropriate to first examine the number of atoms required to form a critical nucleus at the WSL junction as a function of undercooling. There are two effects of increasing undercooling.

(1) As the undercooling is increased, the number of atoms, $n^{*}$, required to form a critical nucleus at the WLS junction decreases, as given by the following relationship:

$$
\mathrm{n}^{*}=\left[\left(\frac{32 \pi}{3 \mathrm{v}_{\mathrm{a}}}\right)\left(\frac{\gamma_{\rho \mathrm{I}}}{\Delta \mathrm{S}_{\mathrm{v}}^{\beta}}\right)^{3} F(\theta, \phi, \eta)\right]\left(\frac{1}{\Delta \mathrm{T}}\right)^{3}
$$

which is shown in Fig. 13.

(2) As the undercooling is increased, more time is available for the clusters to form. It can be shown that the time, $t$, available for cluster formation increases as the undercooling is increased according to the relationship:

$$
t=\frac{\Delta T}{G V}
$$

Thus the number of atoms in a cluster at the WL site will increase with undercooling, in order for the clusters at the wall-liquid site to contain sufficient atoms to form a critical nucleus at the experimentally observed undercooling of $0.66 \mathrm{~K}$, the variation in the number of atoms in the cluster with undercooling should be of the form shown in Fig. 13, such that the two curves intersect at the observed undercooling of $0.66 \mathrm{~K}$.

The key problem here is that the cluster formation, according to the classical model of 
thermal fluctuations, is a very slow process such that the curve for the number of atoms in the cluster will be much lower and would intersect the $n^{*}$ curve at very large undercooling of $96.64 \mathrm{~K}$. Thus, it is quite apparent that the clustering process is enhanced at the wall of the ampoule. This is possible since the atoms in the liquid are attracted to the wall, and the number of atomic layers is small so that the use of equilibrium contact angles is not valid [10, 11]. In fact, it is known that the equilibrium contact angle or the constant interface energy is no longer present when the number of atomic layers is of the order of the width of the interface so that the concept of the Gibbs dividing interface can not be used. It has been shown that the interface energy decreases as the radius of the critical nucleus becomes smaller than the width of the diffuse interface. This has recently been confirmed by phasefield calculations that show that the contact angle decreases and approached a complete wetting condition as the number of atomic layers becomes smaller than the width of the interface [12].

\subsection{Nucleation and microstructure evolution}

Nucleation has been shown to play a critical role in governing the microstructure in peritectic systems. Because of the very small undercooling required, nucleation occurs first at the wall-interface junction, and the subsequent microstructure is governed by the relative rates of spreading of the new phase and the continuing growth of the parent phase. If the lateral growth of the new phase is small, the parent phase will continue to grow with increasing undercooling. If the undercooling reaches the value required for nucleation at the parent phase-liquid interface, nucleation will occur along the interface or at any other heterogeneous sites that will influence the final microstructure. We thus calculate the 


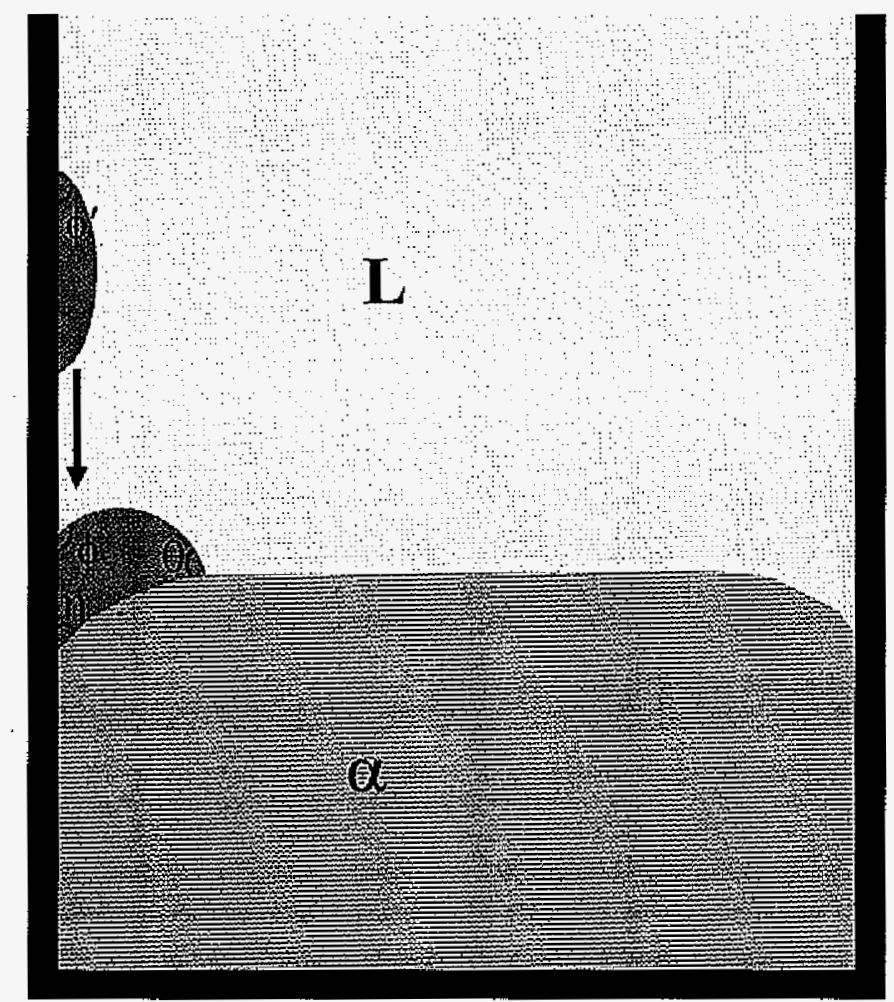

Fig. 12 A schematic illustration of the embryo formation at the wall-liquid interface, which becomes a critical nucleus at the wall-solid-liquid interface when it encounters the interface. 


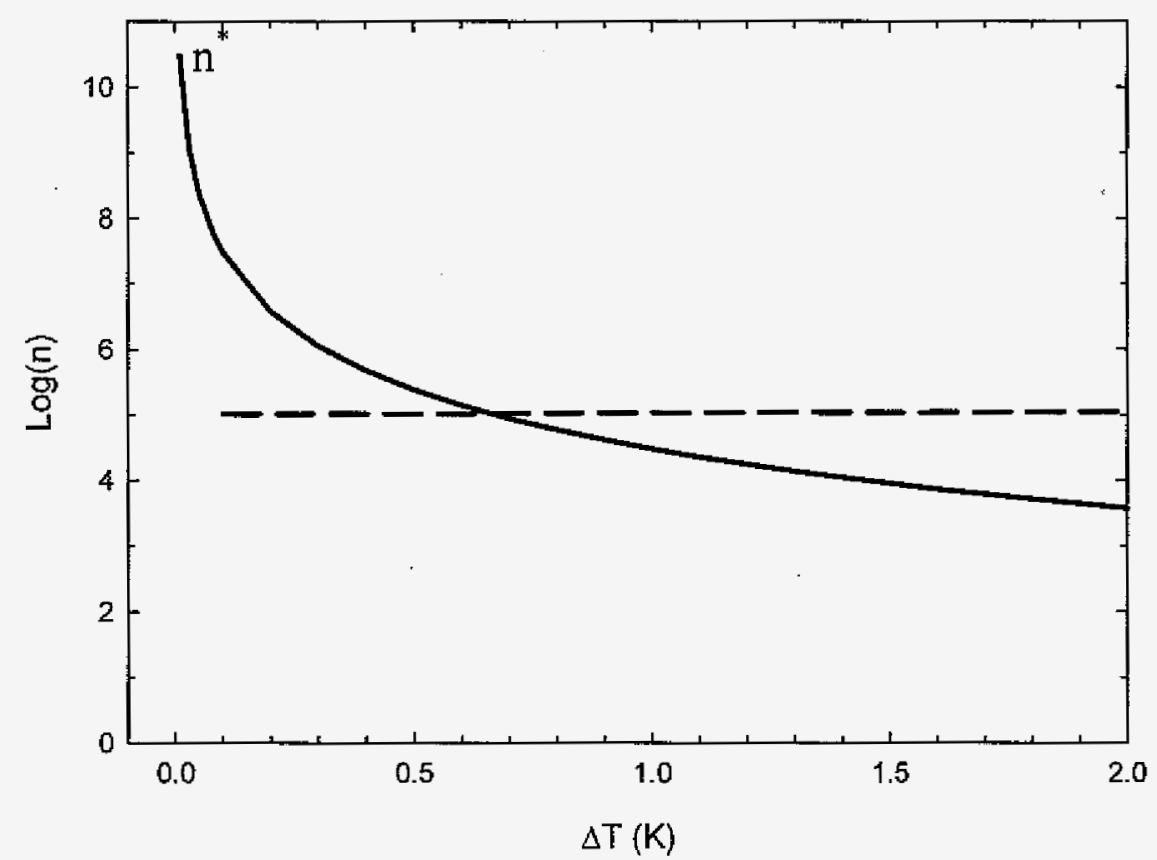

Fig. 13 Number of atoms $\left(n^{*}\right)$ in a critical cluster at the WSL triple junction, and a schematic variation in the total number of atoms in the two subcritical clusters (shown dotted), versus undercooling. 
undercooling values predicted by the thermal nucleation model at different heterogeneous sites by using the measured contact angle values. The predicted undercooling values at different sites are: $96.64,138.3$ and $168 \mathrm{~K}$ at the wall-interface triple junction, parent phaseliquid interface, and the wall-liquid interface, respectively, as shown in Fig. 14. The nucleation undercooling for the homogeneous nucleation of the $\beta$-phase in this system is $203.9 \mathrm{~K}$, which is larger than undercooling required at all the heterogeneous sites.

The value of the nucleation undercooling for the $\beta$-phase plays an important role in the formation of banded microstructures in a peritectic system. The nucleation undercooling of the $\alpha$-phase and the $\beta$-phase determines the composition window over which banding would occur [5], as shown in Fig. 14. To examine this, we fix the undercooling required for the $\alpha-$ phase at the value observed experimentally. This undercooling value determines the upper limit of composition of the banding window. The lower limit of composition for banding is then determined by the $\beta$-phase nucleation undercooling, and this lower limit moves to the larger composition as the nucleation undercooling for the $\beta$-phase is increased. In fact, the banding window will disappear if the undercooling value increases such that this boundary moves to coincide with the upper boundary. This limiting undercooling value for which no banding will occur is shown by an open circle in Fig. 14. The composition range, or the width of the banding window, as a function of the nucleation undercooling for the $\beta$-phase is shown in Fig. 15 that shows that no band formation in the Sn-Cd system would occur if the nucleation undercooling for the $\beta$-phase is larger than about $3.0 \mathrm{~K}$. Since the calculated theoretical values at all heterogeneous sites, based on the classical nucleation model, are significantly larger than $3.0 \mathrm{~K}$, no banding should occur in the Sn-Cd system and the initial 


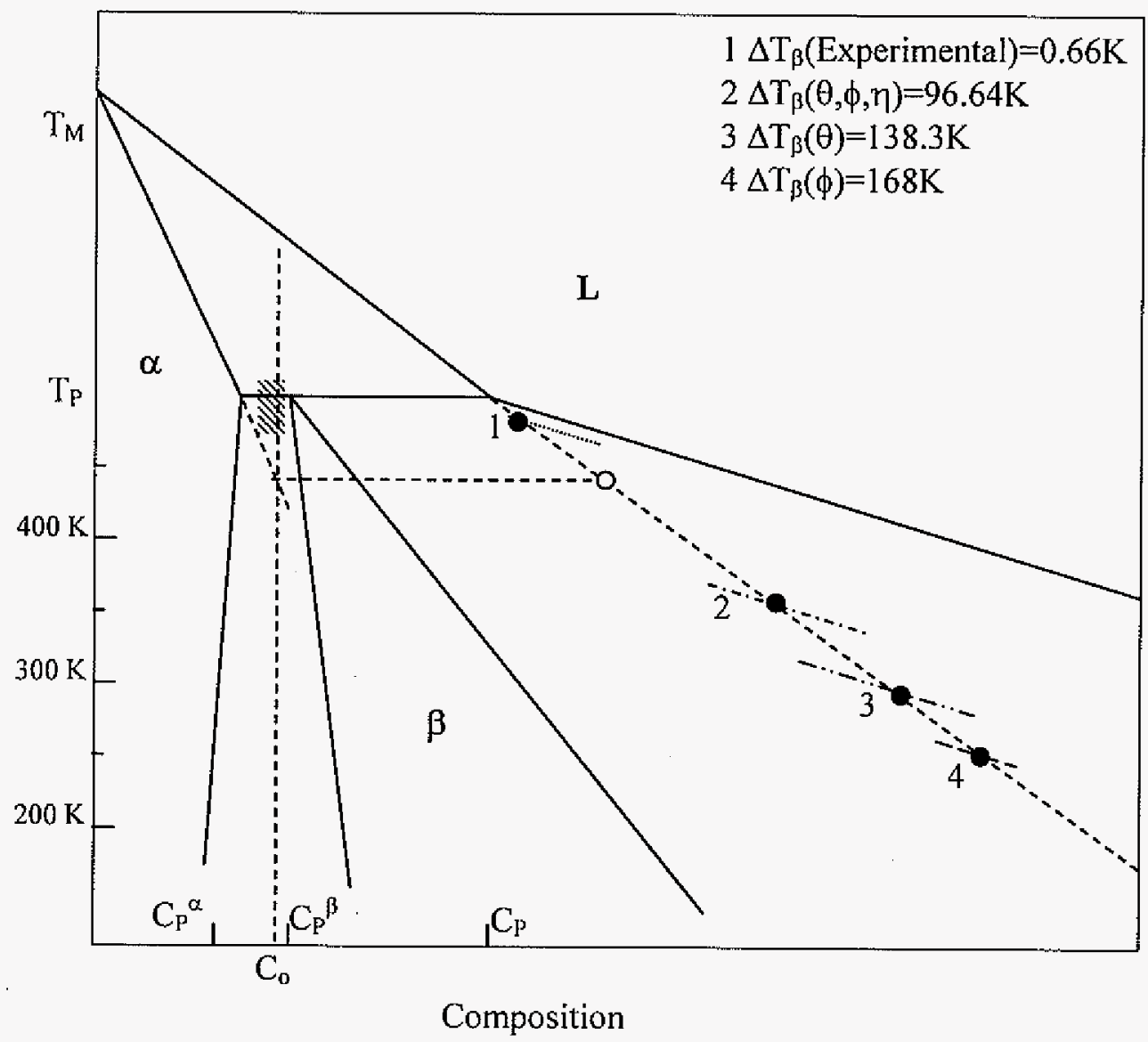

Fig. 14 Calculated nucleation undercooling of the $\beta$-phase at different nucleation sites. The banding window of composition is shown by the shaded region, and the nucleation undercooling at which the banding window will disappear is shown by the open circle. 


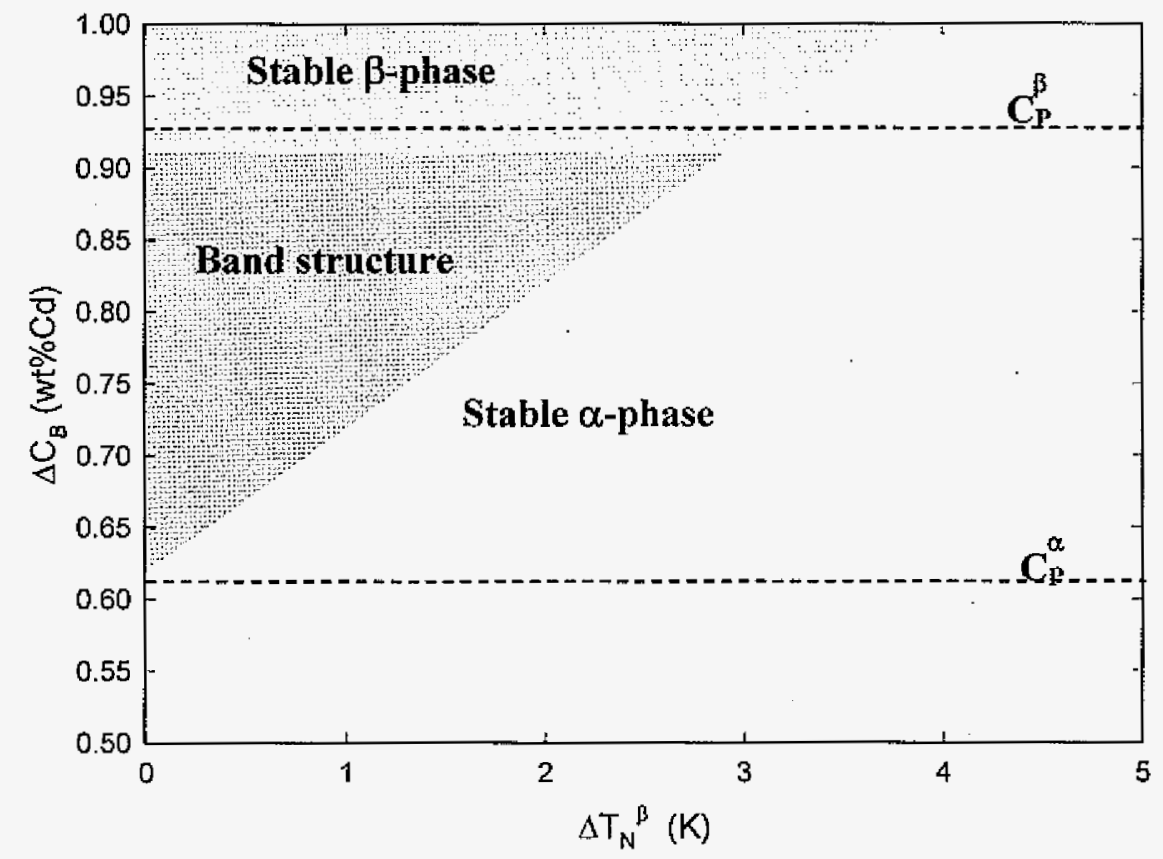

Fig. 15 Microstructure as a function of the amount of nucleation undercooling of the $\beta$-phase under $0.2 \mathrm{~K}$ of nucleation undercooling of the $\alpha$-phase. 
primary $\alpha$-phase will reach steady-state planar front growth condition before any nucleation of the $\beta$-phase can occur. The existence of the banded structure thus emphasizes the importance of the new mechanism presented in this paper.

\section{Conclusions}

Directional solidification experiments in the Sn-Cd peritectic system have been carried out at high thermal gradient to velocity ratio. A banded or an oscillatory microstructure forms by the process of repeated nucleation of the primary and the peritectic phases. Nucleation undercooling for the two phases has been measured from temperature profile and the composition measurements. Nucleation of each phase is observed to occur at the ampoule wall-solid-liquid triple junction, and the results are compared with the classical heterogeneous nucleation model. The experimental undercooling for the two phases has been observed to be more than two orders of magnitude smaller than the theoretically predicted values. It is shown that classical heterogeneous nucleation model is not operative. A new model is presented in which the cluster formation occurs at the wall-liquid interface, and the number of layers in the clusters is smaller than the thickness of the diffused interface. In this case, non-equilibrium contact angle will be present. The clusters are sub-critical for nucleation at the wall-liquid, but they can become critical clusters when they reach the wallsolid-liquid triple junction, since the critical nucleus at the triple junctions contains fewer

atoms. This mechanism requires very small undercooling, which plays a critical role in the development of banded microstructure. 


\section{Acknowledgements}

Appreciation is expressed to Dr. S. Liu for valuable discussions. This work was carried out at Ames Laboratory.

\section{References}

1. J.H. Perepezko and M.J. Uttormark, Metall. Mater. Trans. A, 27A(3) (1996) 533-547.

2. J.H. Perepezko, Mater. Sci. Eng. A, 178A(1-2) (1994) 105-111.

3. R. Trivedi, Metall. Mater. Trans. A, 26A(6) (1995) 1583-1590.

4. R. Trivedi, J.S. Park, J. Cryst. Growth, 235(1-4) (2002) 572-588.

5. R. Trivedi, Scripta mater., 53(1) (2005) 47-52.

6. S. Liu and R. Trivedi, Metall. Mater. Trans. A, 37A(11) (2006) 3293-3304.

7. J.S. Park, and R. Trivedi, J.cryst. growth, 187(3-4) (1998) 511-515.

8. A. Karma, W.J. Rappel, B.C. Fuh, and R. Trivedi, Metall. Mater. Trans. A, 29A(3) (1998) $1457-1470$.

9. S.Liu, Unpublished work, lowa State Univeristy, Ames, IA 50011 (2004).

10. T. E. Quested and A. L. Greer, Acta Metall., 53(9) (2005) 2683-2692.

11. B. Cantor, Phil. Trans R. Soc. Lond., 361 (2003) 409-417.

12. L. Granasy, T. Pusztai, D. Sailor, and J. A. Warren, Phys. Rev. Lett. 98 (2007) 035703/1-4. 
Appendix 1. Contact angle relationships

We consider energy balance at each triple point, shown in Fig. 8. For the nucleation of the $\beta$-phase at the $\alpha$ :L:wall junction, the following conditions need to be satisfied

$$
\begin{aligned}
& \gamma_{\alpha \beta} \cos \eta_{\beta}+\gamma_{W \beta}=\gamma_{W \alpha} \\
& \gamma_{\beta L} \cos \phi_{\beta}+\gamma_{W \beta}=\gamma_{W L} \\
& \gamma_{\beta L} \cos \theta_{\beta}+\gamma_{\alpha \beta}=\gamma_{\alpha L}
\end{aligned}
$$

For the $\alpha$-phase nucleation at the $\beta$ :L:wall junction, we obtain the following conditions

$$
\begin{aligned}
& \gamma_{\alpha \beta} \cos \eta_{\alpha}+\gamma_{W \alpha}=\gamma_{W \beta} \\
& \gamma_{\alpha L} \cos \phi_{\alpha}+\gamma_{W \alpha}=\gamma_{W L} \\
& \gamma_{\alpha L} \cos \theta_{\alpha}+\gamma_{\alpha \beta}=\gamma_{\beta L}
\end{aligned}
$$

The above equations give the following relationships among the contact angles.

(1) from equations (A1a) and (A2a), we obtain $\cos \eta_{\beta}=-\cos \eta_{\alpha}$, or

$$
\eta_{\beta}=\pi-\eta_{\alpha}
$$

(2) From equation $(\mathrm{A} 1 \mathrm{C})$ and $(\mathrm{A} 2 \mathrm{c})$, we obtain

$$
\frac{\gamma_{\beta L}}{\gamma_{\alpha L}}=\frac{1+\cos \theta_{\alpha}}{1+\cos \theta_{\beta}}
$$

(3) From simple algebraic manipulations of equations (A1b) and (A2b), we obtain

$$
\frac{\gamma_{\beta L}}{\gamma_{\alpha t .}}=\frac{\cos \phi_{\alpha}+\cos \eta_{\beta}}{\cos \phi_{\beta}+\cos \theta_{\beta} \cos \eta_{\beta}}
$$

If the contact angles for the $\alpha$-phase are known, then equations (A3-A5) give the corresponding angles for the $\beta$-phase. Thus, only three independent contact angle measurements are needed to assess the validity of the thermally activated nucleation process. However, if it is known that nucleation occurs by the thermally activated process, then two 
additional relationships are obtained for each ampoule material from the experimentally measured nucleation undercooling values, as:

$$
\left[\mathrm{F}_{\beta}(\theta, \phi, \eta)\right]=\left(\frac{14 \Delta \mathrm{S}_{\mathrm{v} \beta}^{2} \mathrm{k} T_{\mu}^{\beta}}{\pi \gamma_{\beta L}^{3}}\right)\left(\Delta T_{N}^{\beta}\right)^{2}
$$

and

$$
\left[\mathrm{F}_{\alpha}(\theta, \phi, \eta)\right]=\left(\frac{14 \Delta \mathrm{S}_{V \alpha}^{2} \mathrm{k} T_{N}^{\alpha}}{\pi \gamma_{\alpha L}^{3}}\right)\left(\Delta T_{N}^{\alpha}\right)^{2}
$$

Consequently, only one angle needs to be measured or estimated for one or more ampoule materials.

For two different ampoule materials, one would need additional four contact angles since the contact angles $\theta_{\alpha}$ and $\theta_{\beta}$ do not depend on the ampoule material. Equation (A3) and (A5) provide two relationships, so that only two additional contact angles in the other ampoule material are required. Thus a total of three angles for one ampoule and a total of five angles for two ampoules are required. 


\title{
CHAPTER 5: COUPLED AND SIMULTANEOUS GROWTH IN PERITECTIC ALLOYS
}

A paper to be submitted to Metallurgical and Materials Transactions

\author{
J. H. Shin and R. Trivedi \\ Materials and Engineering Physics, Ames Laboratory (USDOE) and \\ Department of Materials Science and Engineering, \\ lowa State University, Ames, IA 50011, USA
}

\begin{abstract}
Directional solidification studies were carried out in the $\mathrm{Sn}-\mathrm{Cd}$ peritectic system to study the simultaneous growth of the primary phase ( $\alpha$-phase) and the peritectic phase ( $\beta$ phase) that give rise to a composite microstructure. The mechanism for the evolution of the composite structure was determined by comparing the position of the fronts of the two phases during steady-state growth. Under diffusive growth conditions, a steady-state two-phase composite structure was obtained only when the peritectic phase lagged behind the primary phase. A coupled growth of the two phases, with macroscopically flat interface, was observed when a thin sample was directionally solidified in tantalum ampoules at some specific velocity. The peritectic phase formed along the ampoule wall with the primary phase forming at the center of the ampoule. At velocities slightly away from this specific velocity, the peritectic phase grows along the ampoule wall in an oscillatory manner. It is shown that the coupled growth occurs in thin Ta ampoules due to the constraint of the contact angle at the wall. The rod eutectic model is modified to include the contact angle effect, and the results are shown to agree with the experimental observations.
\end{abstract}




\section{Introduction}

In a peritectic system, a variety of interface patterns can form depending on composition, growth rate and temperature gradient. In the two-phase region of peritectic systems, the microstructures contain both the primary $(\alpha)$ and the peritectic $(\beta)$ phases. These microstructures can be broadly classified into two types [1]: (1) oscillatory microstructures and (2) steady-state composite microstructures, as shown in Fig. I (a) and 1 (b). The growth condition, $G / V$, and the composition range over which these two types of microstructures form are identified as regimes $I$ and $I I$ in the Fig 1 (c) $[1,2]$. The formation of oscillatory microstructures leading to partial or complete band formation has been discussed earlier [1, 3-8]. Here we shall concentrate on the formation of two-phase composite microstructure that forms under experimental conditions defined by the regime II in Fig. 1 (c). The composite structure consists of a two-dimensional array of primary cells or dendrites with peritectic phase in the intercellular or interdendritic region. For primary cells, the composite microstructure appears as a rod eutectic structure in the transverse section behind the interface, and experimental studies have clearly demonstrated that, after some transient, steady-state composite microstructures can be formed over the entire length of the sample [1, 8].

The formation of the peritectic phase in the intercellular region of the primary phase has been discussed by Lograsso et al. [2], who obtained a relationship between the primary and peritectic front positions with velocity, thermal gradient and composition. The distance between the two fronts was shown to decrease as the velocity was decreased at constant thermal gradient and composition. When the composite microstructure forms with peritectic front behind the primary front, we shall refer to this growth mode as the simultaneous growth. 

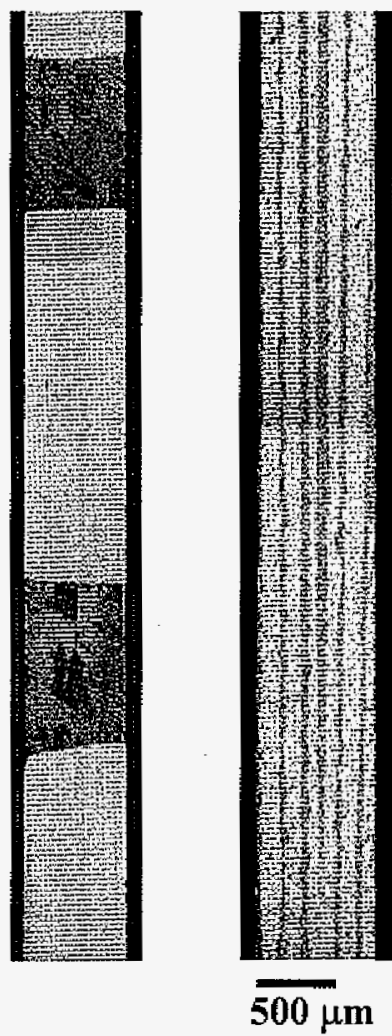

(a)

(b)

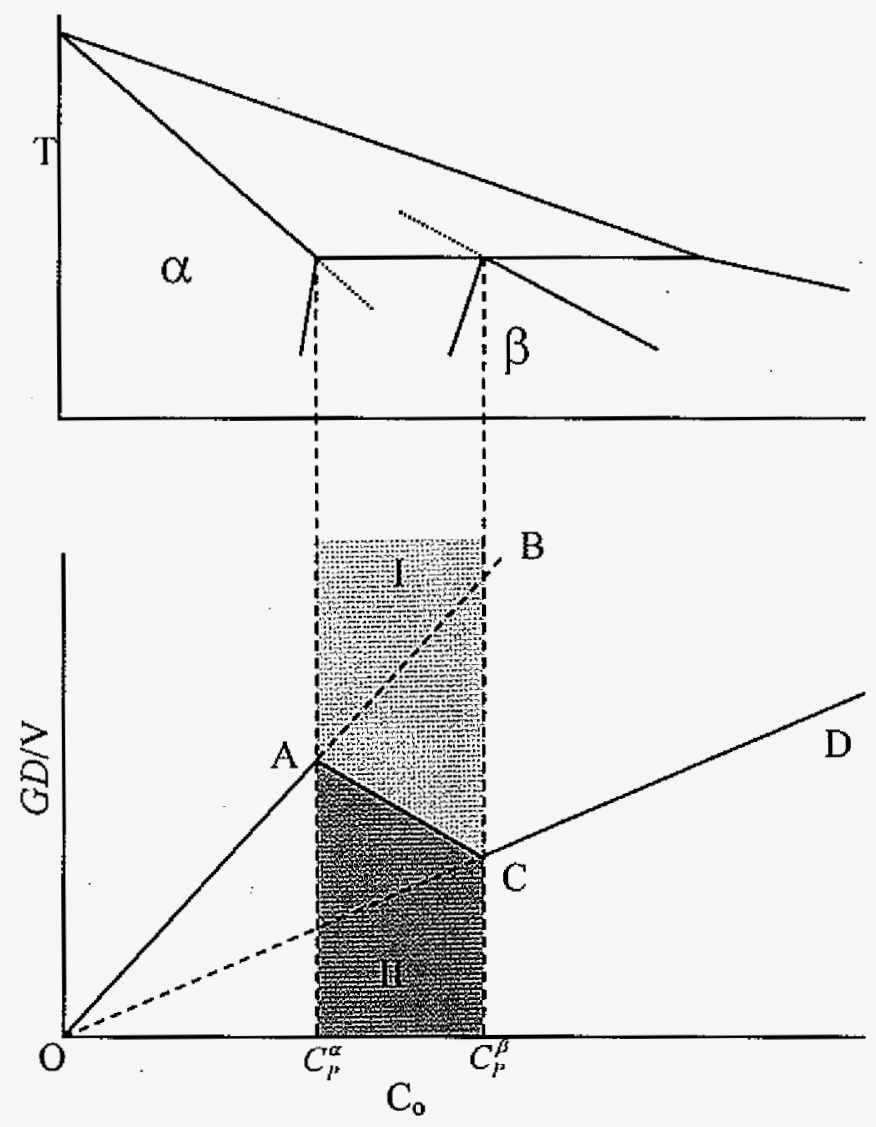

(c)

Fig. 1 (a) A two-phase banded microstructure, (b) a two-phase composite microstructure, and (c) Microstructure map in the two-phase region of the phase diagram showing the regime I for the banded microstructure and the regime II for the composite microstructure [1]. 
Since the difference between the primary and the peritectic fronts decrease with the decrease in velocity, a critical velocity can be defined at which both the phases grow at the same temperature, i.e. the two-interface is at the same temperature. We shall refer to this growth as the coupled growth in analogy with the eutectic growth. One of the critical questions is to establish whether the microstructure consisting of primary rods in a peritectic matrix is stable or not when the two phases grow at the same temperature. Although phase-field simulations have shown the coupled growth in a peritectic system to be stable, no unambiguous experimental verification is available since the model is based on diffusive growth only, while the experiments that show coupled growth have significant convection effects present.

The aim of this study is to carry out critical experiments in the Sn-Cd system under diffusive growth conditions to systematically examine the relative positions of the two phases near the advancing front under different growth conditions. It is found that when the distance between the two fronts becomes small, i.e. below certain critical distance, the rod eutectic type microstructure becomes unstable and no composite growth of primary rods in a peritectic matrix occurs. The second aim of this study is to explore experimental conditions in which a coupled growth could be formed. For this study, directional solidification is carried out in thin samples in three different ampoule materials, viz. quartz, carbon, and tantalum, where the contact angle at the wall will influence the microstructure. It is found that at specific growth conditions, in a tantalum ampoule, the peritectic phase grows along the wall and the primary phase grows in the center, with both the phases growing under steady-state conditions with a macroscopically flat interface. This coupled growth is observed only in the tantalum ampoule, and not in quartz and carbon ampoules, thus indicating the importance of the contact angle at the wall on the stability of a coupled 
microstructure. The model of rod eutectic is modified to incorporate the effect of the contact angle at the wall, and the results are shown to agree with the experimental observations.

The experimental study of the stability of the two-phase microstructure requires careful considerations of the convection effects since they can significantly influence the stability of the interface. We shall first discuss the effect of convection on the formation of a composite microstructure, then examine experimental studies in which convection effects have been establish the alloy system and experimental conditions where significant convection effects have been present, and finally describe experimental technique in which convection effects can be made negligible. We shall then use this technique to study steadystate composite microstructures under diffusive growth conditions.

\section{Convection effects}

Experimental studies in Al-Ni [9, 10], Fe-Ni [11-13], PB-Bi [2] and Sn-Cd [[1, 8] systems have been carried out to examine the formation of a macroscopically planar twophase interface in peritectic systems. The presence of planar two-phase interfaces has been observed by Lee and Verhoeven $[9,10]$ in the Al-Ni system, and by Kurz et al., [11-13] in the Fe-Ni system as shown in Fig. 2. These coupled growth microstructures formed under convective growth conditions, and the role of convection on the stability of the coupled growth needs to be investigated.

In most directional solidification experiments convection effects are generally present that will alter local conditions and may stabilize or destabilize the possible coupled growth. Two types of convection effects are present depending on the whether the rejected solute is lighter or heavier, or whether the density gradient at the interface in the liquid is positive or 


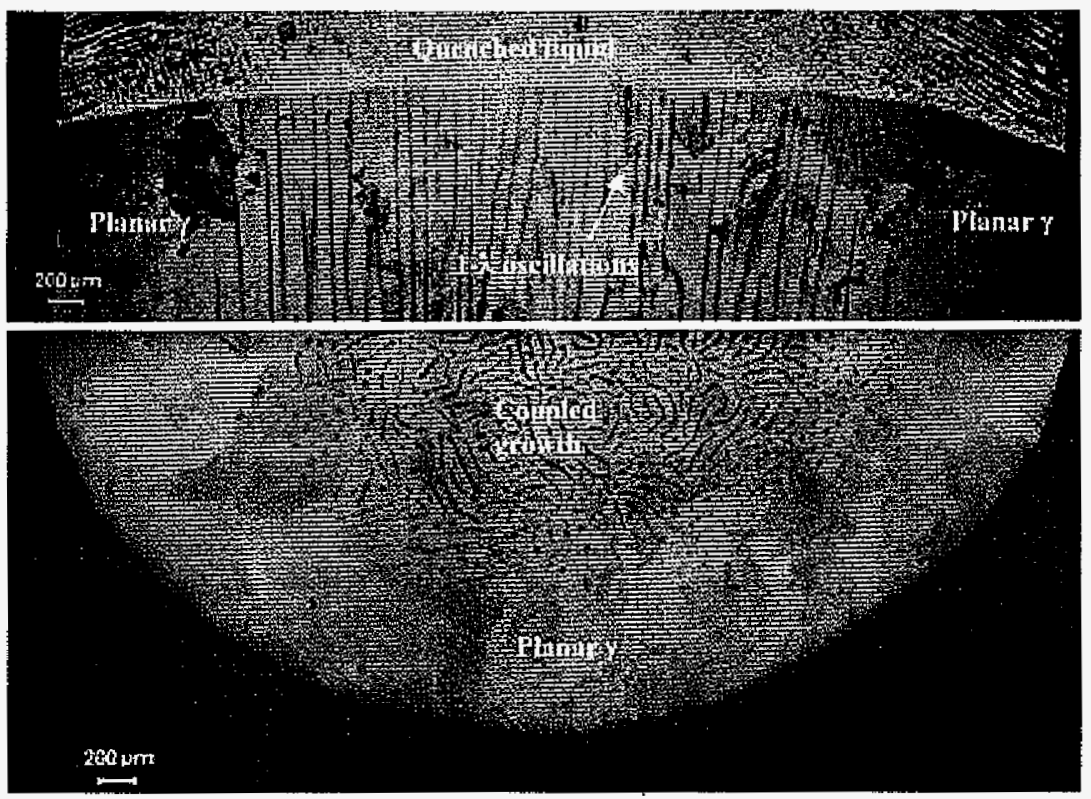

Fig. 2 Coupled growth in Fe-Ni alloy solidified at $\mathrm{G}=18 \mathrm{~K} / \mathrm{mm}, \mathrm{V}=10 \mu \mathrm{m} / \mathrm{s}$. 
negative. When the rejected solute is lighter, a strong double diffusive convection occurs that can give rise to strong oscillating convection $[16,17]$ or partial mixing in the liquid that can be described by the boundary layer models $[18,19]$. These theoretical models have clearly shown that no steady-state growth condition can be achieved since the interface velocity first increases somewhat quickly, and then increases slowly, and then decreases near the end of the sample. Since the velocity is not constant, the distance between the primary and the peritectic fronts will change continuously with solid fraction, so that no steady-state structures can be obtained. In this case, Lograsso et al. [2] have carried out detailed experimental studies in the $\mathrm{Pb}-\mathrm{Bi}$ system, and shown that no steady-state condition was reached, and the peritectic phase that formed behind the primary phase slowly advanced towards the primary front with and then overtook the primary phase as the solidification fraction was increased. Consequently, one may observe the eutectic-like microstructure during the transient conditions at some specific solidification fraction. In fact, when the peritectic phase overtakes the primary phase, a sharp planar two-phase interface in the quenched sample is often observed that should not be confused with the coupled growth. Since the convection effects are quite strong when the rejected solute is lighter, one would require extremely thin sample to minimize convection. Detailed experiments by Liu and Trivedi [7] in $\mathrm{Pb}-\mathrm{Bi}$ system have shown the presence of significant convection even in sample size as small as $0.4 \mathrm{~mm}$. Consequently, any study on coupled growth in a system in which a lighter solute is rejected would give reliable results.

The first requirement for obtaining diffusive growth is to select a system in which a heavier solute is rejected upon solidification. This, however, is not sufficient since any radial temperature gradient in the system will cause fluid flow that will give rise to solute content 
that increases from the center to the ampoule wall $[18,20]$. Since the ampoule is heated from outside in the vertical Bridgman growth, radial temperature variation is always present, and there is no threshold for fluid flow in presence of radial temperature variation. Consequently, fluid flow will also be present in this case such that the concentration will increase radially from the center to the wall. This steady-state variation in composition will also cause microstructure to vary in the radial direction, and the interface will become macroscopically convex. This is clearly seen in the experiments in the Fe-Ni system in which a very clear couple growth is observed in the central region only where the composition will be smaller than the alloy composition $[20,21]$. Since the composition varies in the radial direction, composition gradients in the radial direction at the interface will be present that may influence the stability of the microstructure. Consequently, unambiguous results can be obtained only when the convection effects are made to be negligible.

When the convection effects are due to the radial temperature gradient only, they can be minimized by using a very small diameter sample. The effect of sample diameter on convection is examined in the $\mathrm{Sn}-\mathrm{Cd}$ and $\mathrm{Al}-\mathrm{Cu}$ systems, and the size of the sample required is determined for diffusive growth at a given velocity and for the thermal profile of the directional solidification equipment $[3,4,18]$. Park and Trivedi $[3,4]$ have shown that the convection effects are negligible in the Sn-Cd system at $\mathrm{V}=1.0 \mu \mathrm{m} / \mathrm{s}$ in ampoules of $0.8 \mathrm{~mm}$ diameter. Shin and Trivedi [1] also showed that the convection effects in this system were negligible for velocities of $0.1 \mu \mathrm{m} / \mathrm{s}$ in ampoule of $0.6 \mathrm{~mm}$ diameter. Directional solidification experiments can thus be carried out in the $\mathrm{Sn}-\mathrm{Cd}$ system under diffusive growth conditions. 


\section{Experimental procedure}

Directional solidification experiment was carried out in $\mathrm{Sn}-0.9 \mathrm{wt} \% \mathrm{Cd}$ hypoperitectic alloy. The $G / \mathrm{V}$ ratio in the range of $4 \times 10^{3}$ to $7 \times 10^{3} \mathrm{~K} \cdot \mathrm{s} / \mathrm{mm}^{2}$ was used since the microstructure transition from cellular structure to planar interface is predicted within this range. Experiments were carried out in the bulk tube $(\phi=4.0 \mathrm{~mm})$ and thin tube $(\phi=0.4 \sim 0.6$ $\mathrm{mm})$. Two different tube materials such as the quartz and tantalum were used in order to examine the effect of wall material on the microstructure. After melting of the sample in a bulk tube, the thin tube was inserted into the central axial position of the bulk sample using a graphite holder. After the thin tube was set at the desirable position of the bulk sample, the sample was rapidly solidified up to $20 \mathrm{~mm}$ in order to obtain constant heat flow and the sample was then solidified at a desired pulling rate after interface stabilization.

The solidified microstructure was observed with OM (Optical microscope) and SEM (Scanning Electron Microscope) after etching with a $4 \%$ solution of nitric acid in lactic acid.

\section{Experimental results}

The following experimental results will now be examined. (1) The stability of composite microstructure. This will be examined first with and without the convection effects. Then, the stability of the composite microstructure will be related to the distance between the fronts of the primary and the peritectic phases under diffusive growth conditions. (2) The effect of ampoule material on the stability of a coupled growth. 


\subsection{The stability of composite microstructures}

The transverse and the longitudinal cross-sections of the directionally solidified Sn$0.9 \mathrm{wt} \% \mathrm{Cd}$ alloy at $\mathrm{V}=4.0 \mu \mathrm{m} / \mathrm{s}$ are shown in Fig. 3 (a) and 3 (b), respectively. Fig. 3 (a) shows transverse microstructure in the thin quartz ampoule and in the bulk sample. In both parts, microstructure shows that the $\alpha$-phase is surrounded by the $\beta$-phase. The volume of the $\beta$-phase in the bulk sample, i.e. in the region outside the embedded thin tube, however increases in the radial direction, i.e. from the center to the wall. In the thin tube, where diffusive growth is present, the primary cells are surrounded by the peritectic phase, and the microstructure.appears line a two phase composite microstructure. Note that at this velocity, some cells are just beginning to coalesce in the thin sample, so that the growth conditions are very close to the stability limit of the composite microstructure. The longitudinal microstructure, Fig. 3 (b), however, clearly shows that the two phases are not growing at the same temperature. Rather, the interface in the thin tube and in the bulk show that the $\alpha$-phase is the leading phase and the $\beta$-phase grows in the intercellular region of the $\alpha$-phase. The solute rejected laterally by the $\alpha$-phase is absorbed by the $\beta$-phase, which has a higher solute content. Therefore, both $\alpha$-phase and $\beta$-phase do not grow in a coupled manner. The $\alpha$-phase grows independently from the $\beta$-phase, but the $\beta$-phase growth depends on the solute rejected by the $\alpha$-phase. We shall refer to this growth mechanism as the cooperative growth in contrast to the coupled growth.

If $\ell$ be the distance between the $\alpha$ - and the $\beta$ - fronts, then $\ell$ decreases as $V$ decreases at constant $G$ and $\mathrm{C}_{\mathrm{o}}[2]$. Thus, experiments were carried out at lower velocities to obtain 
smaller values of $\ell$ with a limiting case of $\ell=0$ when both the phases grow at the same temperature.

When the velocity was reduced from 4.0 , the length $\ell$ was found to decrease, and its value was measured. Also, the transverse cross section showed that the composite microstructure starts to become more unstable. At the velocity of $3.0 \mu \mathrm{m} / \mathrm{s}$, the length $\ell$ was found to be zero so that both the phases were growing at the same temperature. The microstructures in the transverse section are shown in Fig. 4 for convective and diffusive growth conditions.

Note that, for diffusive growth, the microstructure no longer has a composite structure, and the two phases, although growing at the same temperature, are not coupled. Thus, it can be concluded that a coupled growth does not occur in the Sn-Cd peritectic system under diffusive growth conditions.

The tip composition in the $\alpha$-phase was measured for each velocity, and it was converted to the tip temperature by using the phase diagram. The tip temperature of the $\beta$ phase was then obtained from the knowledge of $\lambda$ and $G$. The variations in the $\alpha$ - and the $\beta$ phase tip temperatures with velocity are shown in Fig. 5. A rod-like cellular $\alpha$-phase in the $\beta$ phase matrix was observed only for $V>4.0 \mu \mathrm{m} / \mathrm{s}$. A deviation in the regular rod pattern was observed to start at $\mathrm{V}=4.0 \mu \mathrm{m} / \mathrm{s}$, as seen in Fig. 3 (a). More disordered was observed at lower velocities and no rod type microstructure could be identified at $V=3.0 \mu \mathrm{m} / \mathrm{s}$ when both the phases were growing at the same temperature. Note that the isothermal interface was found to form at a temperature above the peritectic temperature. 


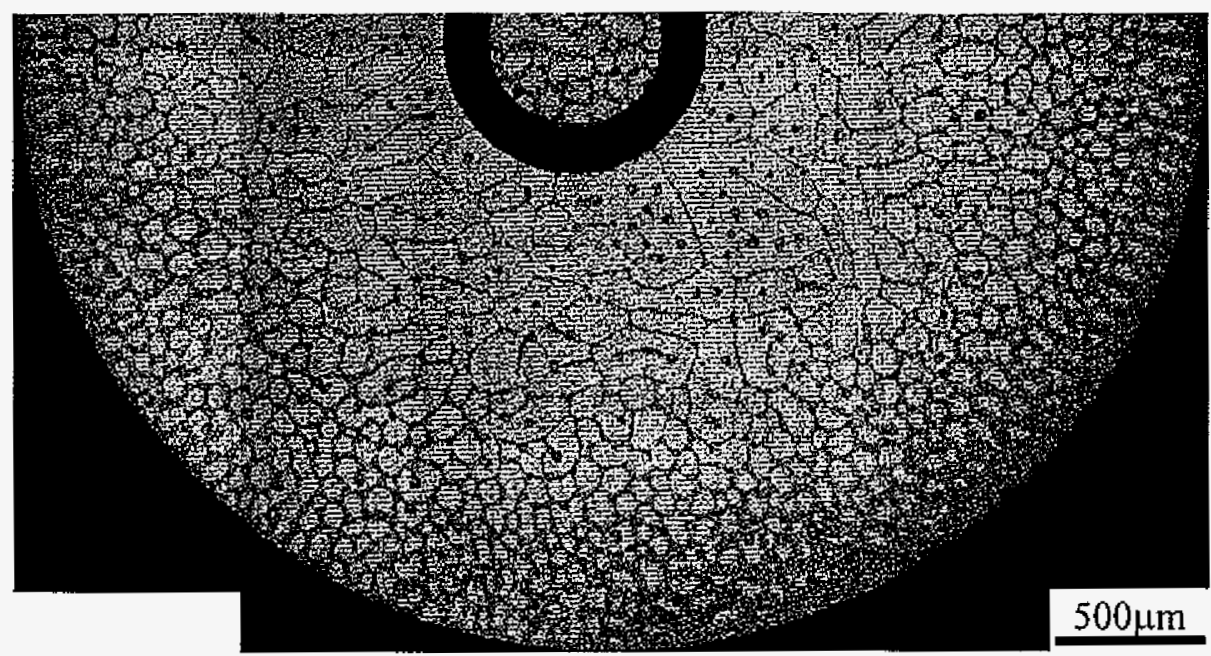

(a)

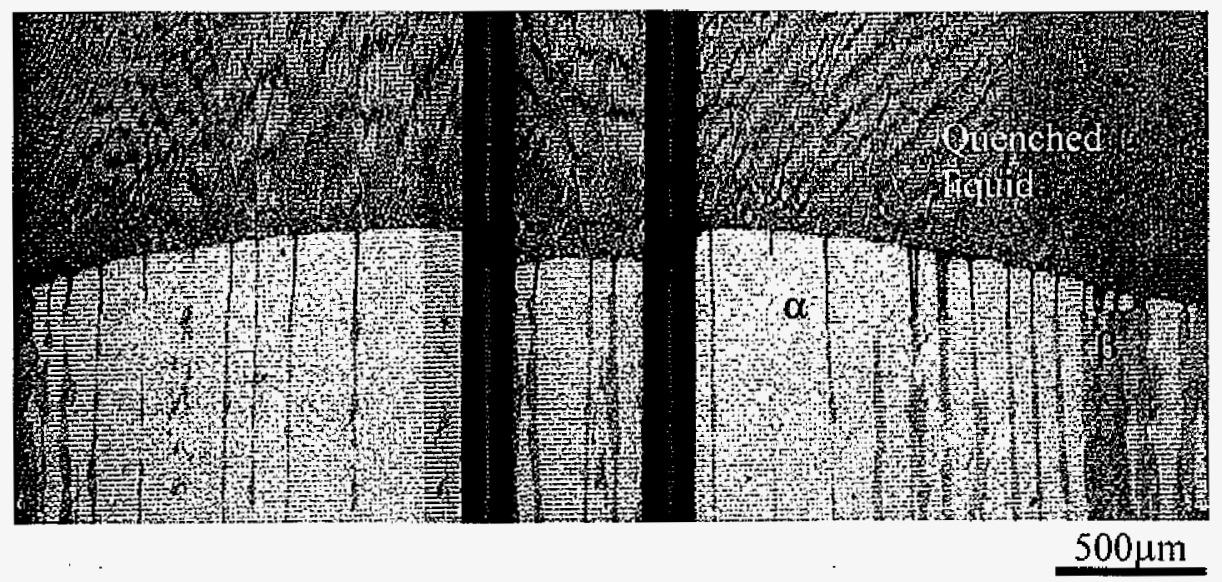

(b)

Fig. 3 Microstructure solidified at $G / V=4.87 \times 10^{3} \mathrm{~K} \cdot \mathrm{s} / \mathrm{mm}^{2}$ in Sn $-0.9 \mathrm{wt} \% \mathrm{Cd}$. (a) Transverse section; (b) longitudinal section. Bright phase is the $\alpha$-phase, dark phase is the $\beta$ phase. 


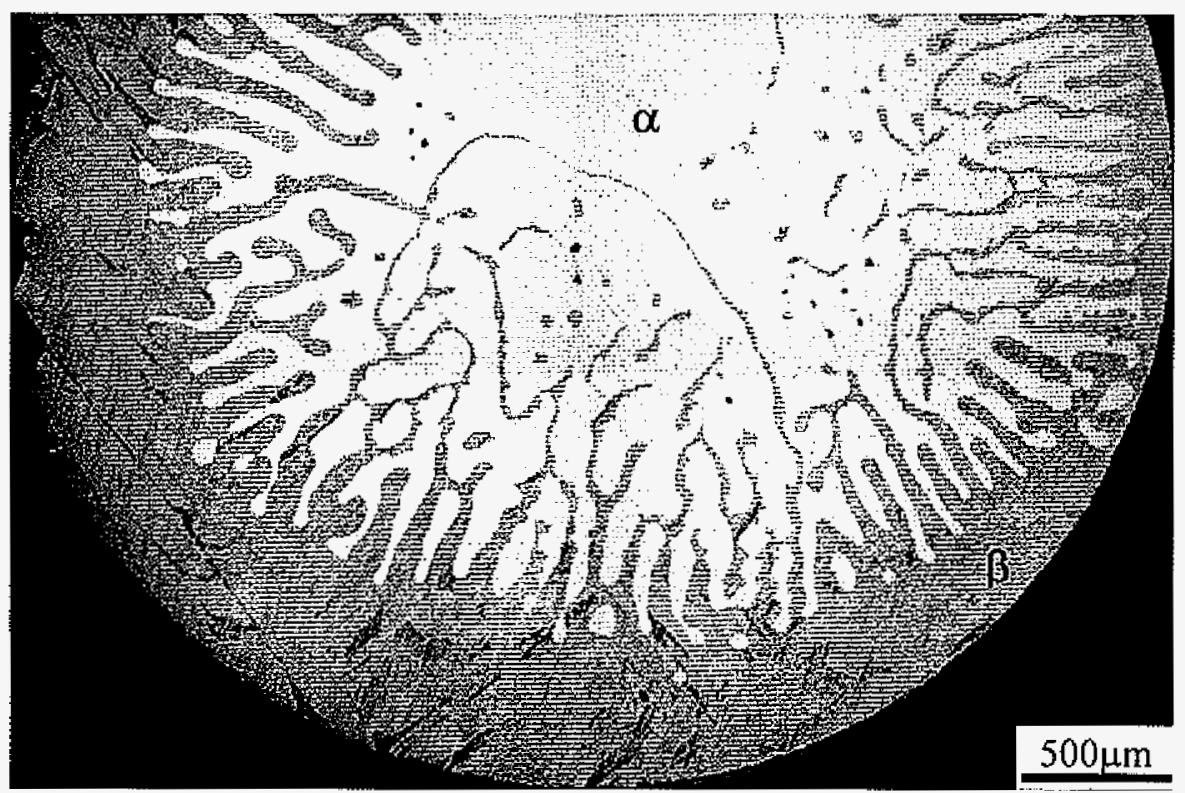

(a)

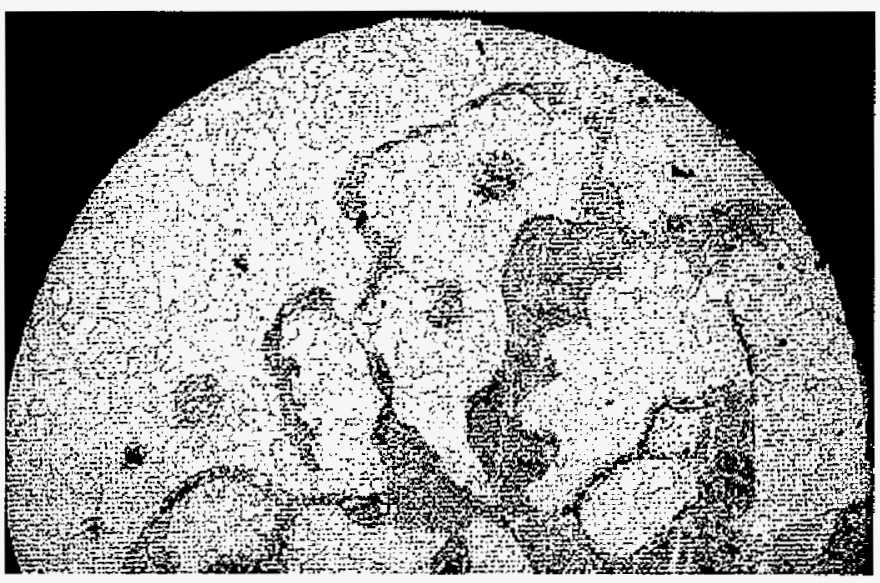

$100 \mu \mathrm{m}$

(b)

Fig, 4 Transverse microstructure solidified at $G / V=6.5 \times 10^{3} \mathrm{~K} \cdot \mathrm{s} / \mathrm{mm}^{2}$ in Sn- $0.9 \mathrm{wt} \% \mathrm{Cd}$.

(a) Convective growth condition; (b) Diffusive growth condition. Bright phase is the $\alpha-$ phase, dark phase is the $\beta$-phase. 


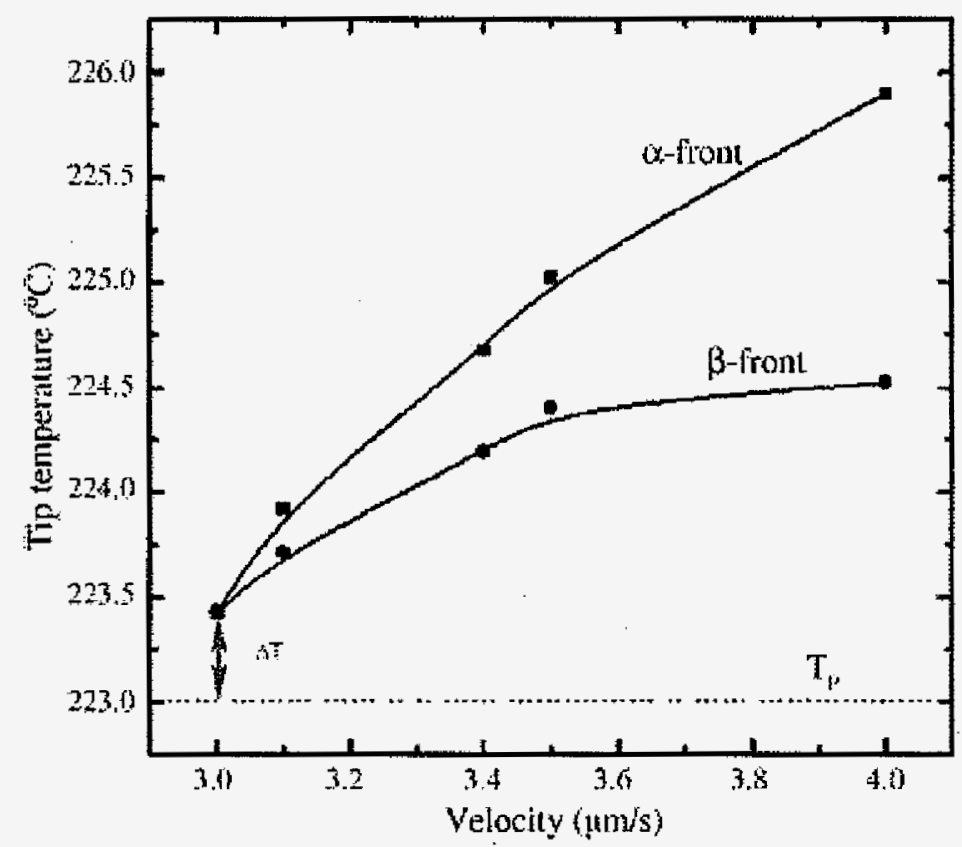

Fig. 5 The variation in the tip temperature of the $\alpha$-phase and the $\beta$-phase interfaces as a function of velocity. 


\subsection{The effect of tube materials on microstructure}

When Sn-Cd alloy was solidified at the quartz tube, microstructure evolves from complete band structure to partial band structure to $\alpha$-cellular structure as growth rate is increased. However, two different microstructures were formed when a hypo-peritectic alloy of Sn-Cd alloy was directionaily solidified in a tantalum tube just above the line AC in Fig. 1. When a Sn-Cd hypo-peritectic alloy was solidified in the tantalum tube under high G/V ratio condition, a complete band structure, as described by Trivedi [22], was observed, as shown in Fig. 6. In the tantalum tube, the $\beta$-phase nucleated at the wall- $\alpha$-liquid junction, and grew rapidly laterally to form a complete band.

However, under a narrow range of $G / V$ ratio, Sn-Cd hypo-peritectic alloy formed a coupled structure with a single rod of the $\alpha$-phase surrounded by the $\beta$-phase, as shown in Fig. 7. The G/V ratio showing coupled growth is just above line $\mathrm{AC}$ in Fig. 1, which shows the limit between band structure and $\alpha$-cell leading composite structure. The criteria of $G / V$ ratio, line $\mathrm{AC}$, for coupled growth under steady state growth condition in hypo-peritectic alloy, is given by

$$
\frac{G}{\mathrm{~V}}=-\frac{m_{\alpha}\left(C_{p}-C_{o}\right)}{D}
$$

In Fig. 7, the $\alpha$-phase forms at the center part of the tantalum tube, and grows at a steadystate, while the $\beta$-phase grows along the wall of the ampoule under steady-state growth conditions. Some curvature of $\alpha$-phase was observed as shown in Fig. 7, but the $\alpha$-rod interface is close to a planar rather than a cellular interface. Both the $\alpha$-phase and the $\beta$-phase do not grow precisely as the steady state growth, but small oscillations in the volume fraction are observed during growth. In fact, as we shall examine later, steady-state growth occurs 
only at one specific velocity for given $G$ and $\mathrm{C}_{\mathrm{o}}$ values. If the velocity is slightly larger or smaller, an oscillatory microstructure is obtained. This was observed experimentally when the velocity was altered.

To identify growth temperature of both phases, composition was measured along the center part of the $\alpha$-rod from the initial position, and the $\beta$-phase composition was measured below the quenched interface. It is found that the $\alpha$-phase grows above the peritectic temperature, whereas the $\beta$-phase grows just below the peritectic temperature, as shown in

Fig. 8. There is interface temperature difference between the $\alpha$-phase and the $\beta$-phase by 3.5 $\mathrm{K}$. This temperature difference, obtained from composition measurement, matches well with that calculated values by the thermal gradient $(G)$ and the distance $(l)$ between the $\alpha$-phase and the $\beta$-phase, as shown in Fig. 5.

\section{Discussion}

Hypo-peritectic alloys have been reported various microstructure evolutions such as complete and partial band structure in diffusive growth condition, and coupled growth and treelike structure in convective growth condition. This discussion focuses on coupled growth in peritectic alloy.

\subsection{Coupled growth in peritectic alloys}

Kurz et al.[11-13] experimentally observed coupled growth in an Fe-Ni alloy under convective growth conditions, as shown in Fig. 2, in which both phases grow at the isothermal interface. Coupled growth in eutectic structure is based on cooperative growth 


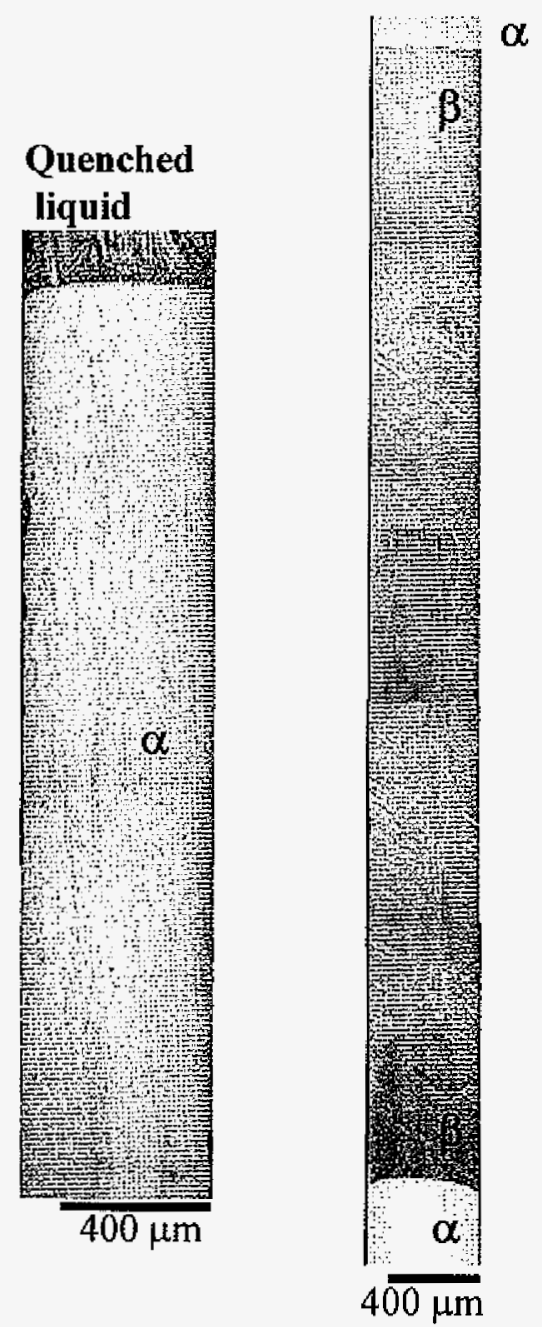

Fig. 6 Complete band microstructure in tantalum tube in Sn-0.9wt\% Cd alloy solidified at $G / \mathrm{V}=19.5 \times 10^{3} \mathrm{~K} \cdot \mathrm{s} / \mathrm{mm}^{2}$ 

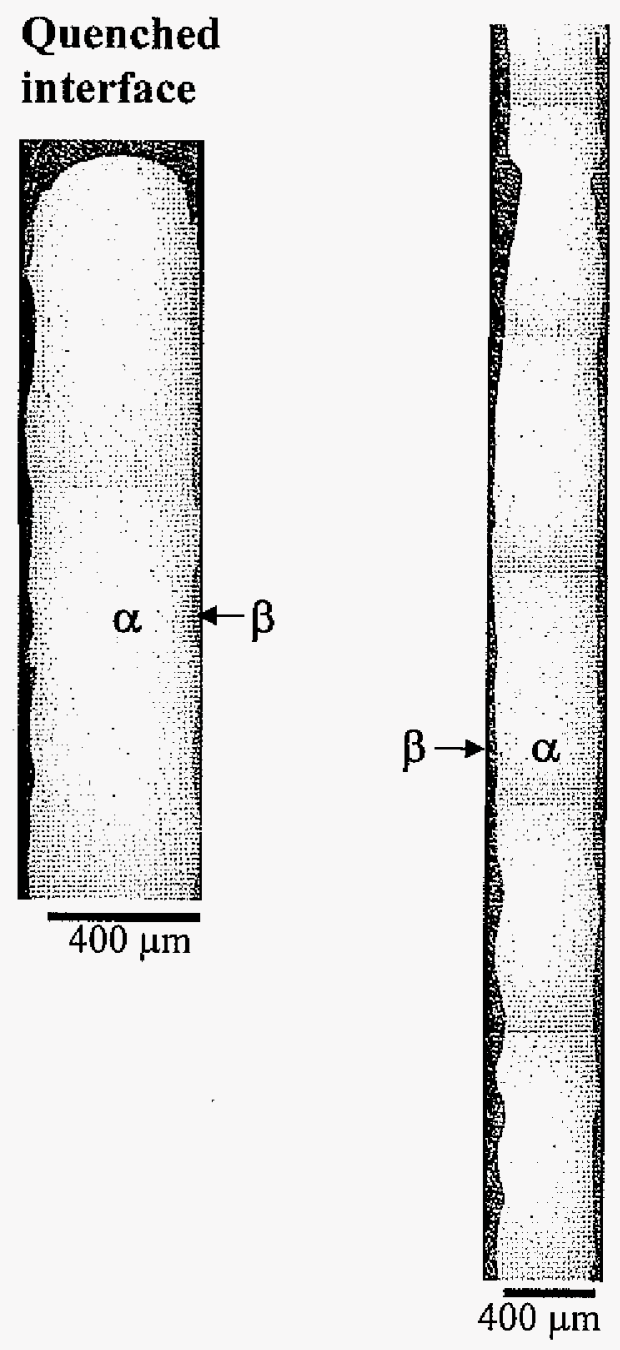

Fig. 7 Rod coupled structure in tantalum tube in Sn-0.9wt\% Cd alloy solidified at $G / \mathrm{V}=7.8 \times 10^{3} \mathrm{~K} \cdot \mathrm{s} / \mathrm{mm}^{2}$. 


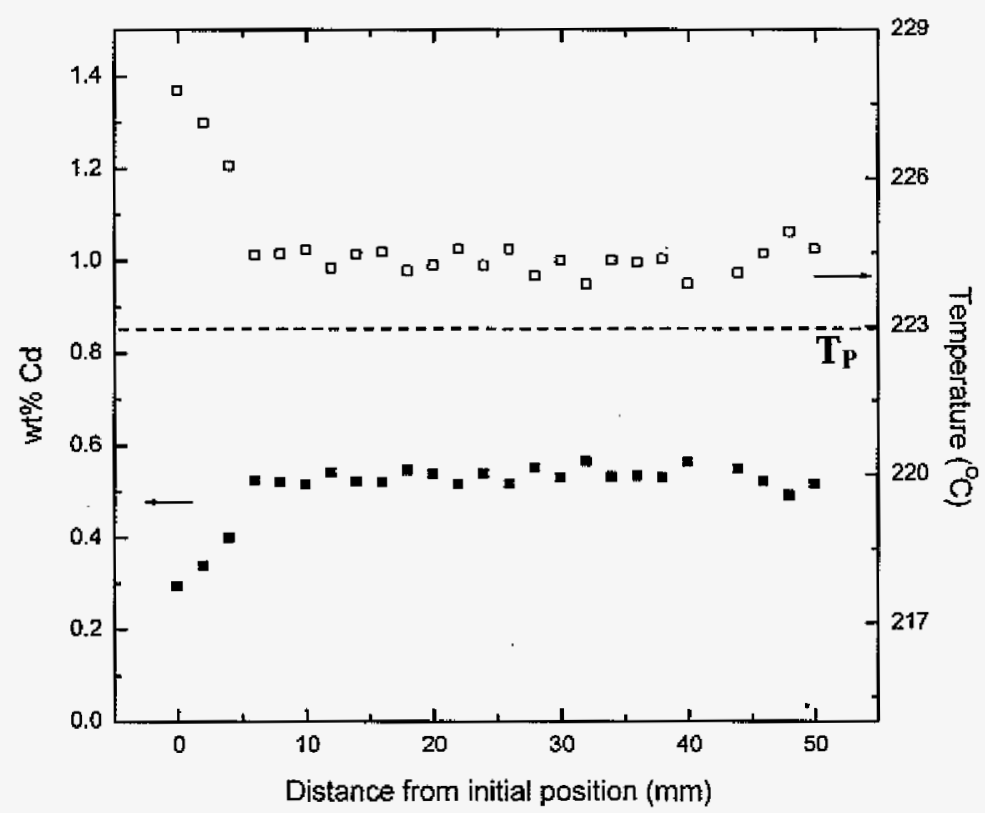

(a)

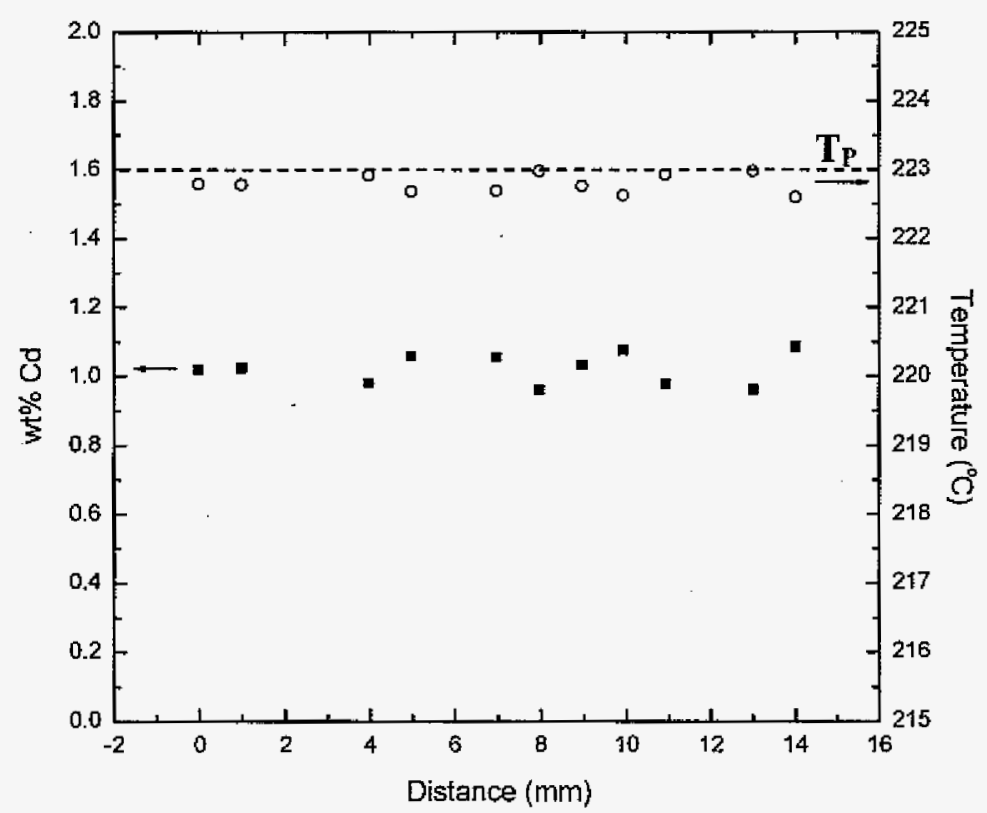

(b)

Fig. 8 Composition and growth temperature of (a) the $\alpha$-phase measured from the initial position and (b) the $\beta$-phase below the quenched interface. 
with exchanging solute and solvent below eutectic temperature since one phase has solute distribution coefficient, $k<1$ and the other phase has $k>1$. In a peritectic system, the $\alpha$-phase and the $\beta$-phase have solute distribution coefficient, $k<1$ so that both phases reject solute at the interface into the liquid. Thus, the two-phase growth in peritectic is somewhat analogous to the eutectic growth in off-eutectic composition in which a long range boundary layer of the solute is present. In order for the eutectic-like growth to occur, some coupling of solute between the two phases is required, i.e. some solute flow from the $\alpha$ - to the $\beta$-phases in the liquid is required. This would require the interface to be above the peritectic temperature where the interface composition in front of the $\alpha$-phase would be larger than that in front of the $\beta$-phase. Although the concentration difference between the liquid ahead of both the phases is present, the solute moving by diffusion along the lateral direction in the liquid is not dominant since both phases reject solute in the liquid and the concentration difference is

very small. Present experimental studies show that the coupling of solute is very weak, and no eutectic-like growth occurs in the Sn-Cd system. Note that both the phases do grow at the same temperature, but the microstructure does not consist of rods of the primary phase in a peritectic matrix. We now examine the coupled growth in a tantalum ampoule.

\subsection{Coupled growth of Sn-Cd alloy in the tantalum tube}

Our experimental results, for the first time, show the formation of a coupled microstructure in a thin tube of tantalum. When Sn-Cd alloy was solidified in the tantalum tube, the $\alpha$ - and the $\beta$-phases grew together and formed a coupled structure, showing a leading $\alpha$-phase with rod shape and the $\beta$-phase growing at the outer edge of the $\alpha$-phase 
with a significant curvature as shown in Fig. 7. The high curvature in the $\alpha$-rod is driven by solute segregation in the lateral direction since solute diffuses from the $\alpha / l$ interface with a high concentration region to the tube wall with a low concentration region.

Coupled growth was not observed in the quartz tube under various $G / V$ ratios, whereas coupled growth was observed only in the tantalum tube. When the $\beta$-phase is nucleated at the w- $\alpha-1$ junction, it grows preferentially along the wall, and the lateral spreading is negligible. Thus as the $\beta$-phase grows along the wall, the $\alpha$-phase keeps growing at the center until it reaches the steady-state growth condition. The stability of this structure is thus governed by the contact angle at the wall.

The effect of contact angle can be examined by considering the two-phase growth by using the approach of Jackson and Hunt $(\mathrm{J}-\mathrm{H})$. J-H model [24] considered 3-D solute diffusion by assuming that the interface can be considered to be planar for the solution of the diffusion equation. A solutal undercooling is thus calculated from the solution of the diffusion equation, and then interface energy contribution is examined that will give an isothermal interface. In the present case, the diffusion undercooling will not be altered, but the average curvature of the $\beta$-phase-liquid interface will change due to the constraint of the contact angle at the wall. If $\phi_{\beta}$ is the contact angle at the wall- $\beta$-liquid junction, then the average curvature is obtained as:

$$
\left\langle\frac{1}{\rho_{\alpha}}\right\rangle=\frac{2}{r_{\alpha}} \sin \theta_{\alpha}
$$

and 


$$
\left\langle\frac{1}{\rho_{\beta}}\right\rangle=\frac{2}{\mathrm{r}_{\alpha}} f_{\mathrm{R}}\left(\sin \theta_{\beta}+\sin \phi_{\beta}\right)
$$

Using these values, and assuming growth at the minimum undercooling, we obtain

$$
\begin{aligned}
& \lambda^{2} V=\frac{K_{2}}{K_{1}} \\
& K_{\mathrm{l}}=\frac{m P C_{o}}{f_{\alpha} f_{\beta} D} \\
& K_{2}=2 m \delta\left(\frac{\Gamma_{\alpha} \sin \theta_{\alpha}}{\left|m_{\alpha}\right| f_{\alpha}}+\frac{\Gamma_{\beta}\left(\sin \theta_{\beta}+\sin \phi_{\beta}\right)}{\left|m_{\beta}\right| f_{\beta}}\right)
\end{aligned}
$$

Where, $m=\frac{\left|m_{\alpha}\right| \cdot\left|m_{\beta}\right|}{\left|m_{\alpha}\right|+\left|m_{\beta}\right|}, P=2 f_{\alpha} \sum_{n=1}^{\infty} \frac{1}{\gamma_{n}^{3}} \frac{J_{1}^{2}\left(\sqrt{f_{\alpha}} \gamma_{n}\right)}{J_{0}^{2}\left(\gamma_{n}\right)}$, and $\delta=2 \sqrt{f_{\alpha}}$

Schematic drawing of contact angles and spacing is shown in Fig. 9. Since the spacing in the thin sample is fixed by the diameter of the sample, $d$, steady state growth would be obtained only at a fixed velocity given by $V=K_{2} / K_{1} d^{2}$. The magnitude of the fixed velocity, for a fixed ampoule diameter, is governed by the constant $K_{1}$ and $K_{2}$, in which $K_{2}$ contains the contact angle at the ampoule wall. Using the $\lambda-\mathrm{V}$ relationship, equation (4), we can calculate the variation in $\lambda$ as a function of velocity for different values of the contact angle at the wall. The results are plotted in Fig. 10. By taking the point that corresponds to $\mathrm{V}$ $=2.5 \mu \mathrm{m} / \mathrm{s}$ and $\lambda=\mathrm{d}$, we can determine the value of the contact angle that would be present for the alloy and ampoule material used in this study. When the contact angle of the $\beta$-phase with the tantalum wall is $44^{\circ}$, the calculated value is matched very well with the spacing and velocity as shown in Fig. 10. Physical properties of $\mathrm{Sn}-\mathrm{Cd}$ alloy that are used in this calculation is shown in table 1. 


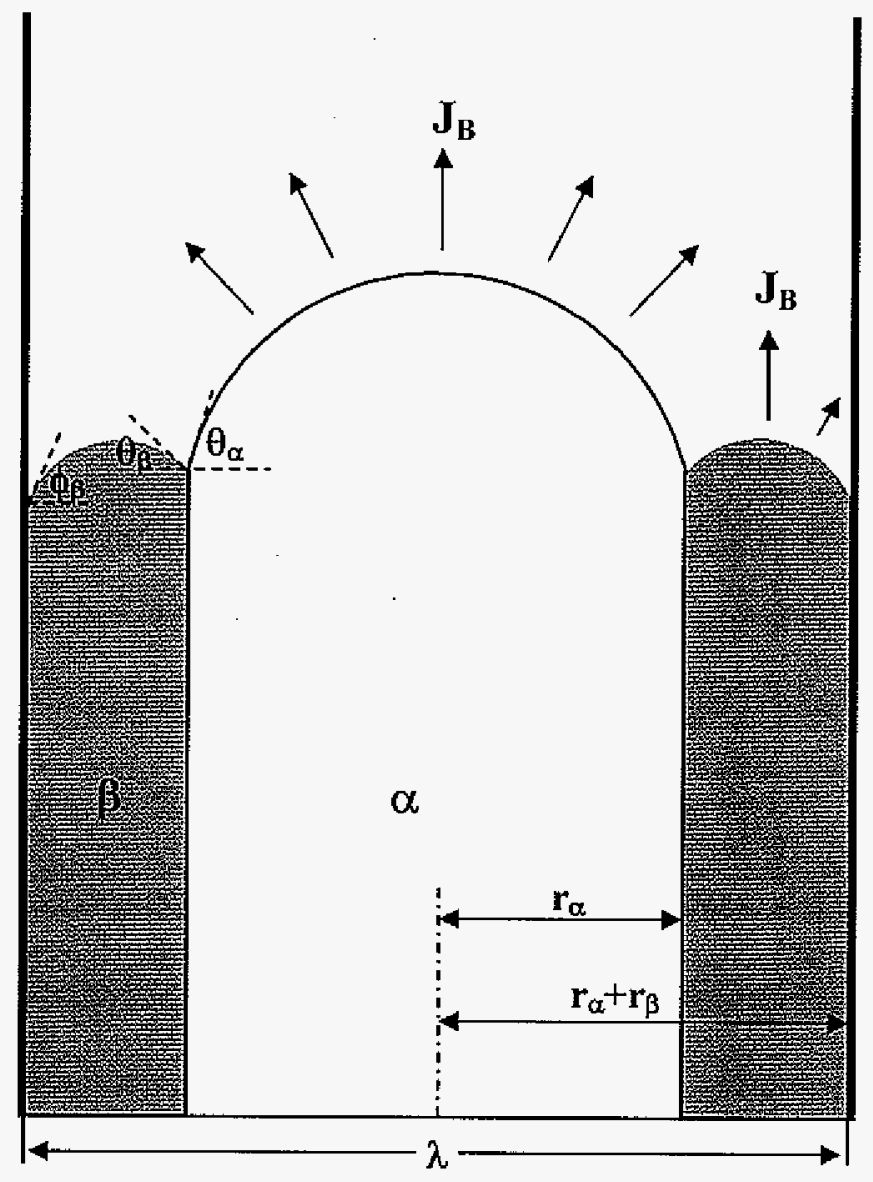

Fig. 9 Schematic drawing of the rod coupled growth showing solute moving, spacing, and the contact angles. 


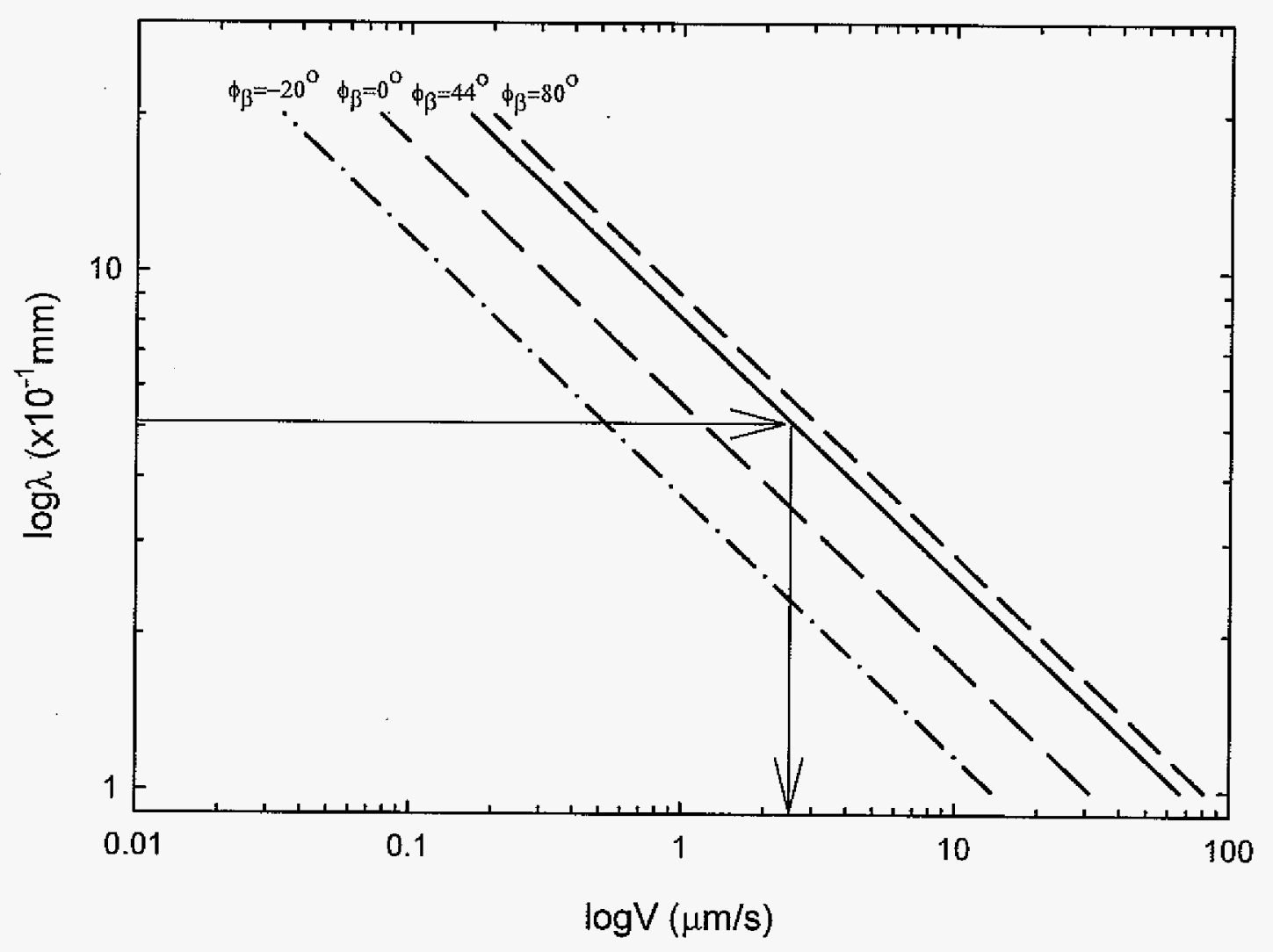

Fig. 10 The variation of the spacing as a function of pulling velocity at each contact angle of the $\beta$-phase contacting with the tantalum tube. 
Table 1 Material properties of Sn-Cd alloy

\begin{tabular}{clc}
\hline Symbol & Value & Unit \\
\hline $\mathrm{m}_{\alpha}$ & -2.368 & $\mathrm{~K} / \mathrm{wt} \%$ \\
$\mathrm{~m}_{\beta}$ & -0.735 & $\mathrm{~K} / \mathrm{wt} \%$ \\
$f_{\alpha}$ & 0.88 & - \\
$f_{\beta}$ & 0.12 & - \\
$\Gamma_{\alpha}$ & $0.9487 \times 10^{-7}$ & $\mathrm{~m} \cdot \mathrm{K}$ \\
$\Gamma_{\beta}$ & $1.073 \times 10^{-7}$ & $\mathrm{~m} \cdot \mathrm{K}$ \\
$\theta_{\alpha}$ & 75 & $\circ$ \\
$\theta_{\beta}$ & 35 & $\circ$ \\
$\mathrm{D}$ & $1.6 \times 10^{-9}$ & $\mathrm{~m} / \mathrm{s}$ \\
$\lambda$ & $0.514 \times 10^{-3}$ & $\mathrm{~m}$ \\
\hline
\end{tabular}


For a fixed contact angle and fixed ampoule diameter, only a fixed velocity gives a steady-state solution. Thus, if a slightly different value of velocity was used, no steady-state growth will be observed. Our experimental studies show that with small deviations in the velocity, an oscillating $\beta$-phase microstructure is observed. In addition, certain contact angle is required to obtain steady-state solution over the range of growth conditions examined. Thus we find that a couple growth occurs in the tantalum ampoule.

\section{Conclusions}

Experiments were carried out to study coupled growth in Sn-Cd peritectic alloy under convective and diffusive growth conditions. Coupled growth was not observed in Sn-Cd alloys under convective growth condition, in which tree-like structure or $\alpha$-cell. leading structure was observed. However, coupled growth in Sn-Cd alloy was found in the tantalum tube. The constraint of the contact angle at the wall was shown to stabilize the coupled growth. For a given ampoule diameter, there is a fixed velocity that will give a coupled growth and this velocity is shown to be determined by the contact angle at the wall.

The contact angle at the wall gives rise to an asymmetry in the diffusion field near the wall since the slope is equal to the tangent of the contact angle rather than zero, which is present in a periodic eutectic structure. Note that convection effects also result in a solute flow in the radial direction, which will also give rise to an asymmetry in the diffusion field, and may influence the stability of the coupled peritectic in bulk samples. Note hat convection not only alters the local composition, but it also gives rise to a composition gradient parallel to the interface, so that eutectic growth in presence of this addition flow of solute must also be considered. Furthermore, critical experiments are required in the $\mathrm{Ni}-\mathrm{Al}$ and $\mathrm{Fe}-\mathrm{Ni}$ systems 
under diffusive growth conditions to establish if coupled peritectic is stable under diffusive growth conditions.

\section{Acknowledgements}

This work was supported by the Office of Basic Energy Science, Division of Materials Science, U. S. Department of Energy under Contract No. W7405-Eng-82.

\section{References}

1. R. Trivedi and J. H. Shin, Mater. Sci. Eng. A, $413-414$ (2005) 288-295.

2. T. A. Lograsso, B. C. Fuh, and R. Trivedi, Metall. Mater. Trans. A ,36A(5) (2005) 12871300.

3. R. Trivedi and J. S. Park, J. Cryst. Growth, 235(1-4) (2002) 572-588.

4. J. S. Park and R. Trivedi, J. Cryst. Growth, 187(3-4) (1998) 511-515.

5. K. Tokieda, H. Yasuda, and I. Ohnaka, Mater. Sci. Eng. A, 262(1-2) (1999) 238-245.

6. P. Mazumder, R. Trivedi, and A. Karma, Metall. Mater. Trans. A, 31A(4) (2000)12331246.

7. S. Liu and R. Trivedi, Metall. Mater. Trans. A, 37A(11) (2006) 3293-3304.

8. W. J. Boettinger, Metall. Trans., 5(9) (1974) 2023-2031.

9. J. Lee and J. Verhoeven, J. Cryst. Growth, 143(1-2) (1994) 86-102.

10. J. Lee and J. Verhoeven, J. Cryst. Growth, 144(3-4) (1994) 353-366.

11. S. Dobler, T.S. Lo, M. Plapp, A. Karma, and W. Kurz, Acta Mater., 52(9) (2004) 2795 2808.

12. M. Vandyoussefi, H. W. Kerr, and W. Kurz, Acta Mater., 48(9) (2000) 2297-2306. 
13. T.S. Lo, S. Dobler, M. Plapp, A. Karma, and W. Kurz, Acta Mater., 51(3) (2003) $599-$ 611.

14. G. B. McFadden and S. R. Coriell, Phys. Fluids, 30(3) (1987) 659-671.

15. S. R. Coriell, M. R. Cordes, W. J. Boettinger, and R. F. Sekerka, J. Cryst. Growth, 49(1) (1980) 13-28.

16. P. Mazumder and R. Trivedi, Appl. Math. Mod., 28 (2004) 109-125.

17. P. Mazumder, R. Trivedi, and A. Karma, Metall. Mater. Trans., 31 A(4) (2000) 1233.

18. R. Trivedi, P. Mazumder and S.N. Tewari, Metall. Mat. Trans. 33A(12) (2002) 3763.

19. A. Karma, J-W Rappel, B. C. Fuh and R. Trivedi, Metall. Mater. Trans., 29A(5) (1998) $1457-1470$.

20. J.H Lee, S. Liu and R. Trivedi, Metall. Mater. Trans., 36A(11) (2005) 3111-3125.

21. J. D. Verhoeven, J. T. Mason, and R. Trivedi, Metall. Trans. A, 17A(6) (1986) 99I-1000.

22. R. Trivedi, Metall. Mater. Trans. A, 26A(6) (1995) 1583-1590.

23. M, sumida, Mater. Trans., 44(7) (2003) 1351-1358.

24. K. A. Jackson and J. D. Hunt, Trans. Metall. AIME, 236(8) (1966)1129-1142.

25. R. Trivedi and W. Kurz, Solidification Processing of Eutectic Alloys, ed. By G. J. Abbaschian, D. M . Stefanescu, and R. J. Bayuzik. Warrendale, PA: The Materials Society 1988 . 


\section{CHAPTER 6: GENERAL CONCLUSIONS}

When two phases form during the solidification of alloys, the development of microstructure can be governed by the complex interactions of nucleation and growth competition. The evolution of complex microstructure is examined through well-designed experiments in the two-phase region of hypo-peritectic alloys to isolate the effects of nucleation and growth competition. To obtain diffusive growth, experiments are carried out in capillary ampoules of diameters less than $0.6 \mathrm{~mm}$. Two sets of experiments were carried out, and analyzed theoretically.

Directional solidification experiments were carried out at high $G D / \mathrm{V}$ ratios where both the primary and the peritectic phases would grow with a planar interface. Oscillatory microstructures are observed in which bands of the primary and the peritectic phases form alternately in the growth direction. The periodicity of the banded structure, and the composition range over which the bands form is related to the nucleation undercooling of the two phases. Experimentally, nucleation was found to occur at the wall-solid-liquid interface, and the undercooling was found to be very small for each phase. A model for heterogeneous nucleation at the wall-solid-liquid was used to very the experimental results. However, undercooling for heterogeneous nucleation at the wall-solid-liquid junction was found to be only a fraction of $1^{\circ} \mathrm{C}$, which is order of magnitude smaller than the value predicted by the classical nucleation theory. It is proposed that clustering of atoms in the liquid occur at the wall, analogous to the adsorption on a substrate, due to the interaction between the wall and atoms in the liquid. In this case, the clusters near the wall form with very few layers that are less than the thickness of the interface, and a non-equilibrium contact angle will be present 
whose value can go to zero as the number of layers approaches one. When this small contact angle cluster meets the wall-liquid-solid interface, it can become a critical size embryo since fewer atoms are required for the critical nucleus size at this location. In essence, an embryo of subcritical size at the wall-liquid interface becomes a critical size nucleus as at encounters the wall-solid-liquid junction. It is shown that the mechanism for very small nucleation undercooling is critical for microstructure formation, since the higher mucleation undercooling predicted by the classical nucleation model will not form bands or oscillatory microstructures.

Some of the fundamental aspects of the formation of composite, complete and partial bands, and mixtures of these microstructures have been developed to understand the mechanism of microstructure formation under diffusive growth conditions in the two-phase region of peritectic alloys. A microstructure map is developed for composite and oscillatory band formation in peritectic alloys as a function of thermal gradient, velocity, and composition.

The formation of composite microstructures, such as rod eutectic, is examined experimentally in the Sn-Cd system. The conditions under which a steady-state growth occurs are established in thin samples in which diffusive growth is present. It is found that the peritectic phase lags the primary phase at the growth front, and the composite microstructure becomes unstable when dome critical distance is reached between the primary and the peritectic fronts. Thus, the two-phase composite growth does not occur with a planar front consisting of the two phases, as in the eutectic growth. Our experimental studies in thin tubes of $\mathrm{Ta}$, however, shows the presence of a coupled growth, which is shown to be stabilized by the contact angle at the wall of Ta. A critical contact angle is required at the 
wall since no coupled growth was observed in the quartz and the carbon ampoule. A high contact angel of the peritectic phase with the Ta tube wall stabilizes coupled growth, in which the contact angle of peritectic phase with the Ta tube is $44^{\circ}$. A theoretical model of rod eutectic was modified to include the effect of the contact angle at the wall that required nonzero slope of the peritectic phase at the wall. Since the eutectic spacing is fixed by the ampoule diameter, steady-state growth occurs at only one velocity. When the velocity is changed, no steady-state growth occurs, and the peritectic phase forms an oscillatory structure along the wall. 


\title{
APPENDIX: DETERMINING SOLUTE DIFFUSION COEFFICIENT BY DIRECTIONAL SOLIDIFICATION TECHNIQUE
}

\author{
J. H. Shin', Shan Liu ${ }^{2}$, J. H. Lee ${ }^{3}$ and R. Trivedi ${ }^{1}$ \\ ${ }^{1}$ Department of Materials Science and Engineering, \\ Iowa State University, Ames, IA 50011 \\ ${ }^{2}$ Materials and Engineering Physics Program, Ames Laboratory-USDOE, Ames, IA 50011 \\ ${ }^{3}$ Department of Materials Science, Changwon National University, Kyungnam, Korea
}

\begin{abstract}
The solute diffusion coefficient in the liquid is difficult to reliably determine since convection is inevitable for solidification processes conducted terrestrially. By directionally solidifying a sample in a capillary ampoule, we have determined the diffusion coefficient in different alloy systems. If the rejected solute at the growth interface is heavier, the convection effect in a sample $<\phi 1.0 \mathrm{~mm}$ (diameter) becomes negligible and the diffusion coefficient can be derived through the composition measurement in the quenched liquid. Nonetheless, if the solute is less dense, laminar flow still exists in the liquid of a sample $<\phi 1.0 \mathrm{~mm}$ capillary sample. Then the diffusion coefficient must be evaluated by matching the composition profile with an initial transient model incorporating the convection effect. Consistent values are obtained from samples quenched at different solid fractions in $\mathrm{Pb}-\mathrm{Sb}$ alloys.
\end{abstract}




\section{Introduction}

Different techniques have been employed to determine the value of diffusion coefficient $\left(\mathrm{D}_{l}\right)$, which includes thin layer diffusion [ $\left.1-3\right]$, droplet movement in a temperature gradient field $[4,5]$ and the solute boundary layer measurements in a quenched directionally solidified alloy [6-11]. The last method has been extensively used to evaluate the diffusion coefficient in metallic alloys, which involves measuring the composition profile in the quenched liquid ahead of a planar growth interface and fitting it into diffusive models.

However, different values have been reported for a specific binary system. For example, the experimentally determined value of $\mathrm{D}_{\text {/ }}$ in Al-Cu binary alloys varies from 2.4 to $5.5 \times 10^{-9} \mathrm{~m}^{2} / \mathrm{s}[3,6-11]$, which makes it impossible to validate theoretical models. We believe that the major reason for the scatter is due to the liquid convection which changes solute transport ahead of the growth interface [6]. For upward directional solidification, the positive temperature profile along the axial direction makes the melt thermally stable; but melt flow can still occur due to either the solute distribution in the boundary layer or the temperature non-uniformity along the radial direction; therefore it is incorrect to evaluate $D_{\text {l }}$ by naively comparing the measured composition profile with a diffusive model.

In order to obtain the diffusion coefficient $\mathrm{D}_{/}$correctly, there are only two appropriate routes: (1) conducting a directional solidification experiment in the diffusive regime; or (2) analyzing the measured composition profile with a model including convective mass transport. The first one has been applied to a binary alloy with a heavier solute since a capillary sample makes it possible to obtain a diffusive growth process $[6,12,14]$. However for a binary alloy with a lighter solute, liquid convection occurs due to the solute profile in 
the boundary layer $[15,16]$. Thus only the route (2) can be applied to derive the diffusion coefficient in liquid.

In this paper we shall focus on experimental determination of $\mathrm{D}_{l}$ in $\mathrm{Pb}-\mathrm{Sb}$ alloys where convection exists even in a sample of $\phi 0.4 \mathrm{~mm}$. Samples of different diameters were prepared with different solidification fractions and a consistent value of $D_{F}=(5.4 \pm 0.1) \times 10^{-9}$ $\mathrm{m}^{2} / \mathrm{s}$ was derived by comparing the measured composition profile with the transient growth model by Karma et. al. [17].

\section{Experimental procedure}

A typical Bridgman solidification setup was used to conduct the directional solidification and the ratio of temperature gradient $(G)$ over the moving velocity $\left(V_{p}\right)$ was maintained high enough so that a planar front growth should be ensured for different compositions. We have developed a special technique to conduct directional solidification in a capillary ampoule $[6,12,14]$. For $\mathrm{Pb}-2.0 \mathrm{wt} \% \mathrm{Sb}$ alloys, quartz capillary tubes of different diameters, $\phi 0.4,0.8$ and $1.5 \mathrm{~mm}$ were quenched at solidification fraction $f_{\mathrm{s}}=0.3,0.5$ and 0.7 to examine the possible effect of melt convection. The length of a capillary sample is approximately $100 \mathrm{~mm}$.

Composition analysis was performed with WDS-EPMA. In order to improve the accuracy, a standard was prepared with the composition determined through wet chemical analysis. The standard was homogenized and had the same microstructure as the specimen to be analyzed; therefore the error was minimized to be $\pm 0.1 \mathrm{wt} \% \mathrm{Sb}$. 


\section{Experimental results}

\subsection{Morphology of $\mathrm{Pb}-2.0 \mathrm{wt} \% \mathrm{Sb}$}

Effect of solidification fraction We first examined the morphology of quenched interface to ensure that a planar front has been obtained, especially at a high solidification fraction such as $f_{\mathrm{s}}=0.7$. Fig. 1 shows the interface morphology at $\mathrm{V}_{\mathrm{p}}=0.5 \mu \mathrm{m} / \mathrm{s}$ for $f_{\mathrm{s}}=0.3,0.5$ and 0.7 respectively. The diameter of central capillary sample is $\phi 0.4 \mathrm{~mm}$. A few notable morphological features are: (1) a planar interface has been obtained in both the capillary sample and the bulk for all solidification fractions; (2) capillary samples have lower interface positions, and the difference in interface position between a capillary sample and the bulk increases with solidification fraction; (3) the interface in the capillary sample becomes slanted as the solidification fraction increases. It should be emphasized that the interface in a capillary sample is still planar in nature since serial polishing through a capillary sample does not show any cellular instabilities across the interface.

In a bulk sample growth of $\mathrm{Pb}-\mathrm{Sb}$ alloys, Dean, Kerr and Hellawell found that the liquid was nearly well mixed [18]. However, in the capillary sample, due to the increase in ratio of surface area over volume, the liquid flow significantly slows down and has become single-roll laminar. The directional flow of the liquid near the interface carries solute to the downstream side, leading to a non-uniform solute distribution across the growth front and a slanted interface.

Since the bulk grows under nearly complete mixing in the liquid [18] while the convection intensity in a capillary sample is significantly reduced, the solute accumulation rate at the interface of a capillary sample is faster than that in the bulk; therefore the interface 


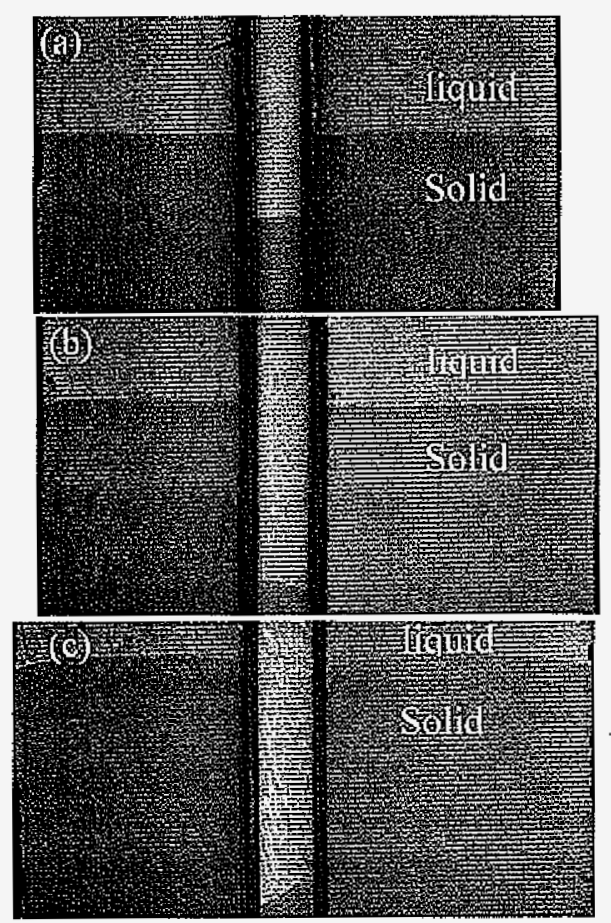

Fig. 1 Interface morphology of $\mathrm{Pb}-2.0 \mathrm{wt} \% \mathrm{Sb}$ alloy quenched at (a) $f_{\mathrm{s}}=0.3$, (b) $f_{\mathrm{s}}=0.5$, (c) $f_{\mathrm{s}}=0.7$ 
position in a capillary sample is always lower and this difference is enhanced with the increase in solidification fraction.

Effect of sample diameter From the above discussion, one may speculate that the interface morphology should also depend on the diameter of a capillary tube. Fig. 2 shows the morphologies of $\phi 0.8$ and $\phi 1.5 \mathrm{~mm}$ capillary samples while the solidification fraction is maintained at 0.5 . These together with Fig. 1 (b) obviously shows the effect of capillary diameter: a larger capillary tube reduces the difference of interface position between the capillary sample and the bulk since it increases the intensity of the liquid convection, reduces the thickness of the diffusion boundary layer ahead of the growth interface and slows down the solute accumulation rate of the interface liquid in the capillary sample. Next, we will present the detailed composition measurement for each sample shown above.

\subsection{Solute distribution}

Composition profiles in the solid and the quenched liquid Fig. 3 shows solute distribution profiles within the solid and the liquid in the $\phi 0.4 \mathrm{~mm}$ capillary sample quenched at $f_{\mathrm{s}}=0.7$. The liquid shows an exponential decrease with distance away from the quenched interface, the solute distribution profile in the solid is rather like that for a diffusive growth process, therefore we will first check whether the initial transient models can describe the measured solute profile or not. The first model is by Smith et. al. [19], which expresses solid concentration $\left(\mathrm{C}_{\mathrm{s}}\right)$ as:

$$
\frac{C_{s}\left(z^{\prime}\right)}{C_{o}}=1+e r f\left(\frac{\sqrt{z^{\prime} V_{p} / D_{L}}}{2}\right)+(2 k-1) \exp \left(\frac{-k(1-k) z^{\prime} V_{p}}{D_{L}}\right) \operatorname{erfc}\left(\frac{2 k-1}{2} \sqrt{z^{\prime} V_{p} / D_{L}}\right)
$$



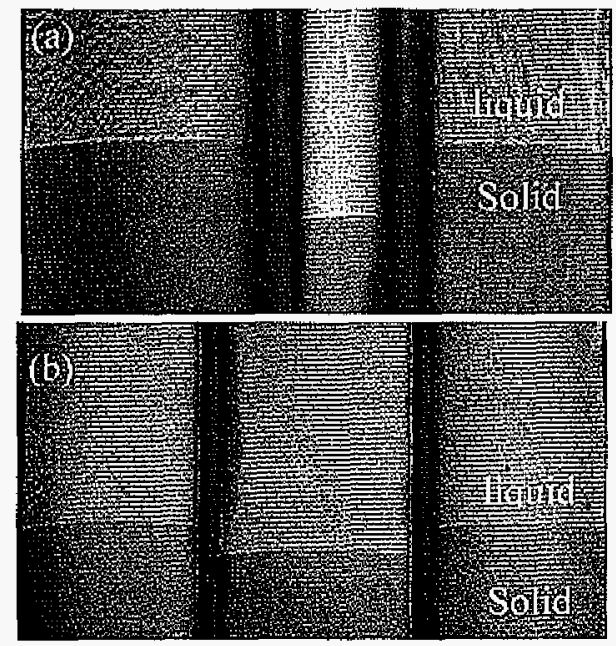

Fig. 2 Interface morphology in (a) $0.8 \mathrm{~mm}$ and (b) $1.5 \mathrm{~mm}$ capillary sample $\left(\mathrm{Pb}-2.0 \mathrm{wt} \% \mathrm{Sb}, f_{\mathrm{s}}=0.5, \mathrm{~V}_{\mathrm{p}}=0.5 \mu \mathrm{m} / \mathrm{s}, \mathrm{G}=17 \mathrm{~K} / \mathrm{mm}\right)$ 
where $\mathrm{C}_{\mathrm{o}}$ is the composition of master alloy, $k$ is the equilibrium solute distribution coefficient, $z^{\prime}$ is the distance from start of solidification. The reported value of diffusion coefficient varies from $(1.0 \sim 6.0) \times 10^{-9} \mathrm{~m}^{2} / \mathrm{s}$, so we choose $D_{F}=(1.0,3.0,6.0) \times 10^{-9} \mathrm{~m}^{2} / \mathrm{s}$ to make the computation. The results are shown in Fig. 3. The profile for $D_{F}=6.0 \times 10^{-9} \mathrm{~m}^{2} / \mathrm{s}$ is systematically lower than the measured composition, the one for $\mathrm{D}_{F}=3.0 \times 10^{-9} \mathrm{~m}^{2} / \mathrm{s}$ overlaps with the measurement up to $50 \mathrm{~mm}$, then the calculation becomes lower than the measured value. For $D_{F}=1.0 \times 10^{-9} \mathrm{~m}^{2} / \mathrm{s}$, the calculated solute accumulation in the first $30 \mathrm{~mm}$ long segment is much higher than the measurement. Therefore, we conclude that the measured composition profile cannot be accounted for by Smith et. al.'s model [19].

We have also made the comparisons with other diffusive growth models such as the one by Favier [20] and similar disagreement has been observed. The fundamental reasons for this disagreement are (1) that convection still exists in the capillary sample, as shown by the $f_{\mathrm{s}}$-dependent microstructures in Fig. 1 , and (2) that all these analytical models assume that the interface velocity instantly jumps to the externally set velocity $V_{p}$ once a sample starts to grow. However, this should not be the case since the instant interface velocity should be controlled by the interface flux balance, which varies in the initial transient. Consequently, a dynamic model such as the one by Karma et. al. [17], which includes this transient velocity effect together with the convection effect in the liquid, should be used to analyze the experimental data.

Effect of solidification fraction The composition in the solid and the liquid has been measured for $\phi 0.4 \mathrm{~mm}$ samples quenched at $f_{\mathrm{s}}=0.3,0.5$ and 0.7 (Fig. 4). One may instantaneously notice that liquid composition at and far from the interface increases with $f_{\mathrm{s}}$. 


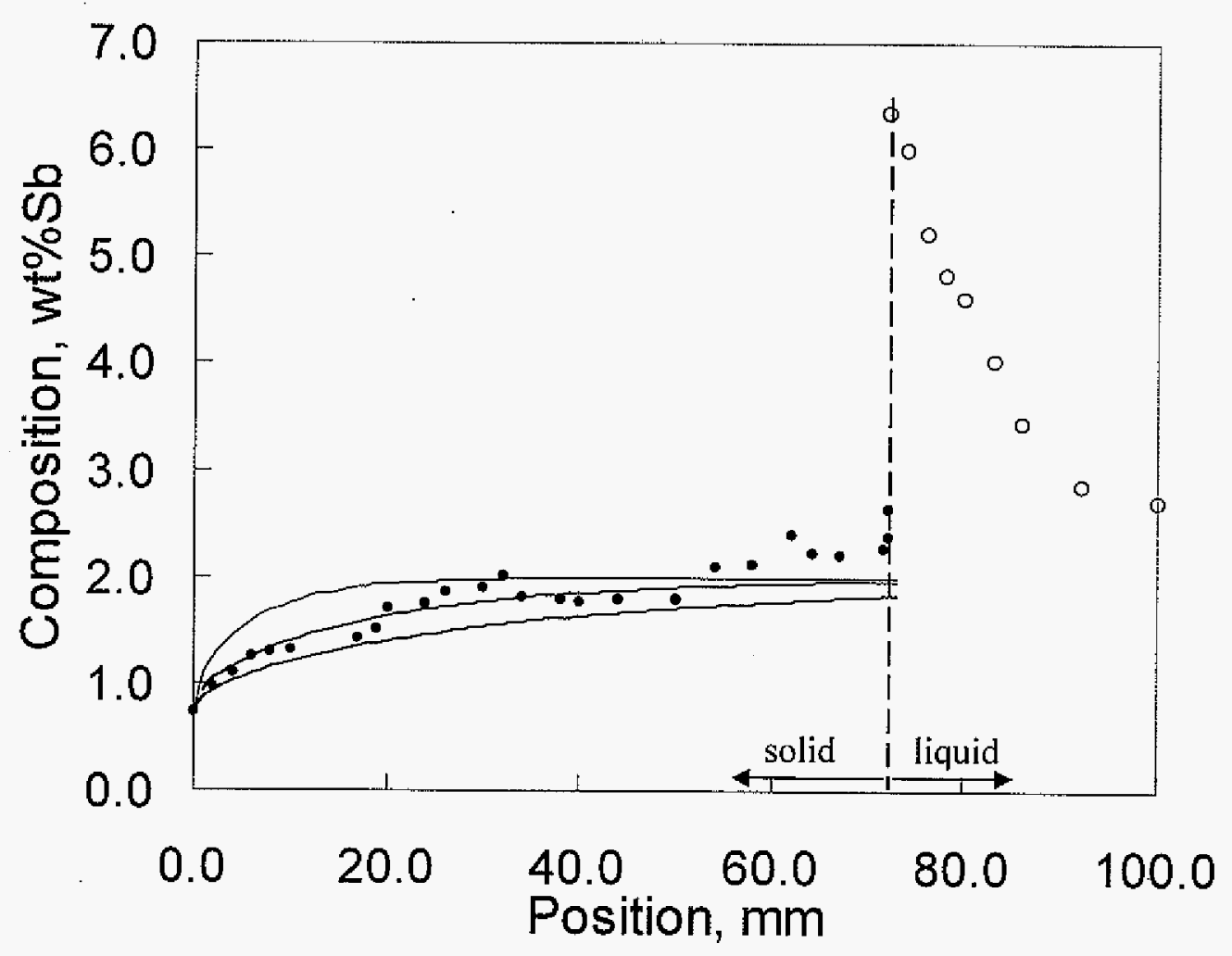

Fig. 3 Comparison of measured solid composition (growth length: $70 \mathrm{~mm}$ ) with calculation of a diffusive growth model [19]. Three lines are for $\mathrm{D}_{F}=1.0 \times 10^{-9}$ (upper curve), $3.0 \times 10^{-9}$ (middle curve) and $6.0 \times 10^{-9} \mathrm{~m}^{2} / \mathrm{s}$ (lower curve) 
More importantly, the composition data in the solid can be considered to overlap on one another since the error related with measurement is $\pm 0.1 \mathrm{wt} \%$. In the context of BPS boundary layer model [21], the boundary layer has the same thickness for these $\phi 0.4 \mathrm{~mm}$ samples despite different solidification fractions.

Effect of capillary diameter Fig. 5 shows the composition measurement in $\phi 0.4, \phi 0.8$, and $\phi 1.5 \mathrm{~mm}$ samples at $f_{\mathrm{s}}=0.5$. The liquid composition profiles are characteristic of the boundary layer flow and a thicker sample has a much narrower diffusion layer. Moreover, the interface composition in a thicker sample is lower and solid composition increases with a slower rate in comparison with a thinner sample. Bulk liquid composition in a thicker sample is also higher. Obviously, these features are closely related with the variation in the intensity of melt flow in samples of different diameters.

\section{Discussion}

\subsection{Calculation of diffusion coefficient}

As mentioned before, the dynamic model by Karma et. al. [17] will be employed to analyze the experimental data and extract the diffusion coefficient in this alloy system. This model has been satisfactorily used in the directional solidification of $\mathrm{Pb}-\mathrm{Bi}$ peritectic alloys and has been found to quantitatively agree with experimental observations regarding the solute distribution and banding formation [16]. In brief, this model includes the following aspects: (1) the sample is of a finite length $L$; (2) the instant interface velocity, $V_{i}(t)$, which is determined by local solute flux balance, increases gradually towards the pulling velocity $V_{p}$; (3) convection is considered in the context of BPS boundary layer model which characterizes 


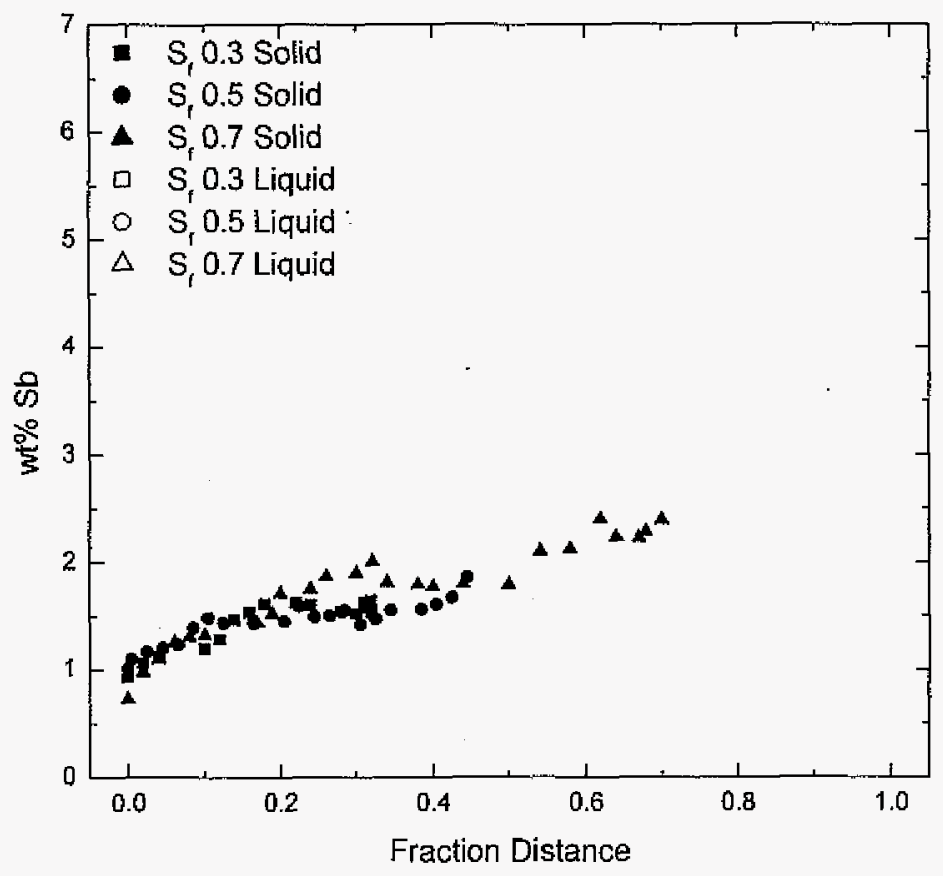

Fig. 4 Measured (symbols) and calculated (lines) composition profiles in the solid and liquid for $f_{\mathrm{s}}=0.3,0.5$ and 0.7 in $0.4 \mathrm{~mm}$ samples of $\mathrm{Pb}-2.0 \mathrm{wt} \% \mathrm{Sb}\left(\mathrm{V}_{\mathrm{p}}=0.5 \mathrm{~m} / \mathrm{s}\right)$ 


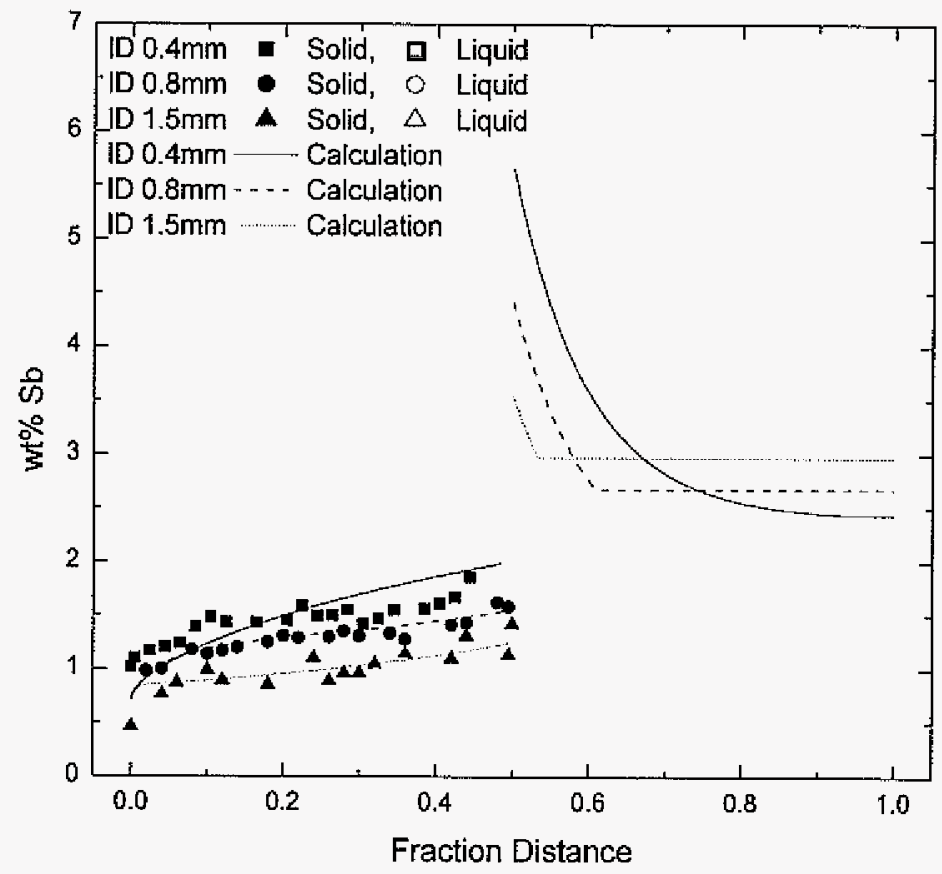

Fig. 5 Measured (symbols) and calculated (lines) composition profiles in the solid and liquid $\phi 0.4, \phi 0.8$, and $\phi 1.5 \mathrm{~mm}$ samples of $\mathrm{Pb}-2.0 \mathrm{wt} \% \mathrm{Sb}\left(\mathrm{V}_{\mathrm{p}}=0.5 \mathrm{~m} / \mathrm{s}, f_{\mathrm{s}}=0.5\right)$ 
the intensity of convection by a single non-dimensional thickness parameter $\Delta$. The model can be expressed by three coupled first-order differential equations that relate the instant interface velocity $V_{i}(t)$, the instant diffusion length $\delta(t)$ and the composition in the bulk liquid $\mathrm{C}_{\mathrm{m}}(\mathrm{t})$ to the boundary layer thickness $\Delta$, pulling velocity $\mathrm{V}_{\mathrm{p}}$ and system properties.

These differential equations can only be numerically solved, but the diffusion coefficient and the boundary layer thickness must be known beforehand. Since evaluating the value of diffusion coefficient is the primary objective of this paper, we have developed an iterative procedure so that the most appropriate values of $\mathrm{D}_{l}$ and $\Delta$ can be found which fit the solute distribution in both the solid and the liquid in different capillary samples. The numerical calculations have already been included in Fig. 4 and Fig. 5. For $\phi 0.4 \mathrm{~mm}$ samples (Fig. 4), $\Delta=4.0$ and $\mathrm{D}_{l}=5.4 \times 10^{-9} \mathrm{~m}^{2} / \mathrm{s}$ best fit the experimental measurement with respect to the solid and liquid composition profiles, and the compositions at the interface. For the $\phi 0.8 \mathrm{~mm}$ sample, with the same diffusion coefficient, $\Delta=1.0$ best fits the experimental data; while for the $\phi 1.5 \mathrm{~mm}$ sample, $\Delta=0.3$ produces a satisfactory agreement (Fig. 5).

It has been suggested that a value of $\Delta=5$ produces a composition profile very close to a solidification controlled by diffusion $[15,20]$; therefore the mass transfer in the $\phi 0.4 \mathrm{~mm}$ sample is close to a diffusive growth process so that the solute distribution in the solid shows the similar feature to a diffusion controlled process.

It should be emphasized that the numerical calculation depends on the selection of $\Delta$ value, a parameter most difficult to estimate. Until now, there is only one analytical expression that can be used to calculate the boundary layer thickness for the Czochralski process where forced convection is introduced by crystal and/or crucible rotation [22]. For a 
Bridgman solidification process, only natural convection occurs, where the flow field is impossible to describe analytically at present, therefore the only method that $\Delta$ has been reported is through analyzing solid composition profile and fitting it with the boundary layer model [23]. In this study, we used both solid and liquid composition profiles in the $\phi 0.4 \mathrm{~mm}$ sample with three solidification fractions to evaluate $\Delta$ and $D_{l}$, and subsequently $\Delta$ values for the larger ampoules were obtained from fitting corresponding composition profiles. Therefore the derived values of $\mathrm{D}_{l}$ and $\Delta$ are considered to be reliable.

\subsection{Comparison with literature data}

There are few published results that report the evaluation of boundary layer thickness $\Delta$ and the diffusion coefficient in $\mathrm{Pb}-\mathrm{Sb}$ system. Dean, Kerr and Hellawell [18] conducted directional solidification in $\mathrm{Pb}-1.0 \mathrm{wt} \% \mathrm{Sb}$ alloys in a $\$ 6.0 \mathrm{~mm}$ glass ampoule aligned horizontally (the sample length is $100 \mathrm{~mm}$ ) and they reported that $\Delta=0.2$ for $V_{p}=1.5 \mu \mathrm{m} / \mathrm{s}$ and $\mathrm{G}=8 \mathrm{~K} / \mathrm{mm}$. Since their sample was horizontal and the sample diameter was much larger than the ones in our study, a thinner boundary layer thickness was very reasonable. They also noticed that slightly increased with growth velocity and sharply increased once the solid/liquid interface was destabilized into cells, then they derived that $\mathrm{D}_{\Gamma}=3.0 \times 10^{-9} \mathrm{~m}^{2} / \mathrm{s}$. However, they used the alloy composition to make calculations, which was not appropriate since liquid composition at the interface continuously increased so that the interface would become destabilized as the solidification process continued. Therefore local interface composition should be used to analyze the interface stability rather than the master alloy composition $[12,14]$. Since liquid composition at the interface was definitely larger than the 
alloy composition, a larger diffusion coefficient than the reported value should be expected. In the present study, we employed the dynamic model for the initial transient and compare solute profiles in both the solid and the liquid. Actually, this diffusion coefficient has been used to analyze the cell/dendrite spacing and dendrite tip radius and a satisfactory agreement has been obtained, which will be reported later.

Chen et. al. [24] studied the macrosegregation behavior in $\mathrm{Pb}-2.0 \mathrm{wt} \% \mathrm{Sb}$ alloys in ampoules of $\phi 1.0,2.0,3.0$, and $7.0 \mathrm{~mm}$. They fit the solute segregation profile with Favier's model [20] and reported that $\Delta=0.8 \phi^{0.53}$. As discussed before, Favier assumed that the interface velocity jumped to the external velocity $V_{p}$ instantly, but the actual interface velocity is smaller than $V_{p}$, which can cause significant deviation for the calculated boundary layer thickness since a slower interface velocity will offer longer time to diffuse rejected solute and increase bulk liquid composition $\mathrm{C}_{\mathrm{m}}(\mathrm{t})$. A careful examination of the comparison with Favier's model (Fig. 6 in [24]) shows that the measured composition for $f_{s}>0.4$ is actually higher than the model prediction.

\subsection{Comparison with determination of $\mathrm{D}_{l}$ in $\mathrm{Al}-\mathrm{Cu}$ alloys}

Since AI-Cu alloys contain a heavier solute, it will be interesting to compare the diffusion coefficient measurement in $\mathrm{Al}-\mathrm{Cu}$ and $\mathrm{Pb}-\mathrm{Sb}$. We have carried out detailed experimental studies to determine $\mathrm{D}_{/}$in capillary samples of $\mathrm{Al}-\mathrm{Cu}$ hypo-eutectic alloys. Moreover, there are a few studies that report the experimentally determined diffusion coefficient from the composition profiles in directional solidification processes $[3,4,6,8-11$, $25,26]$. All the relevant measurements are summarized in Fig. 6 , which clearly shows that the derived diffusion coefficient increases with sample diameter. Only in samples $<1.0 \mathrm{~mm}$ 
does the derived value of diffusion coefficient become independent of sample size, indicating that it is the real magnitude. Those previous experimental studies with samples $>1.0 \mathrm{~mm}$ actually include the convective mass transfer in the liquid, leading to the increase in the effective diffusion coefficient from the composition measurement.

In comparison with the present study in $\mathrm{Pb}-\mathrm{Sb}$ alloys, a clear distinction exists between a heavier and a lighter solute containing alloy: for the former, a thin enough capillary sample makes the diffusive growth possible and the real diffusion coefficient can be obtained through the composition profile in such a capillary sample; while for the later, it is not possible to obtain diffusive growth since the solute boundary layer ahead of the growth interface drives melt convection and the real diffusion coefficient can only be obtained by comparing the measured composition profile with the one calculated with an initial transient model that incorporates the convection effects.

\section{Conclusions}

Systematic experimental studies have been carried out to evaluate the diffusion coefficient in the liquid by analyzing composition profiles in $\mathrm{Pb}-2.0 \mathrm{wt} \% \mathrm{Sb}$ alloys. The capillary sample $(\phi 0.4, \phi 0.8$ and $\phi 1.5 \mathrm{~mm})$ solidification technique has been used in an effort to obtain diffusive growth conditions; however the quenched interface morphologies unambiguously indicate that convection still exists even in the thinnest sample since morphological features vary with solidification fraction and this is further verified by the disagreement between the composition measurement and the calculation by diffusive models. The measured composition profiles in both the solid and the liquid for samples of different solidification fractions and diameters are subsequently compared with the dynamic growth 


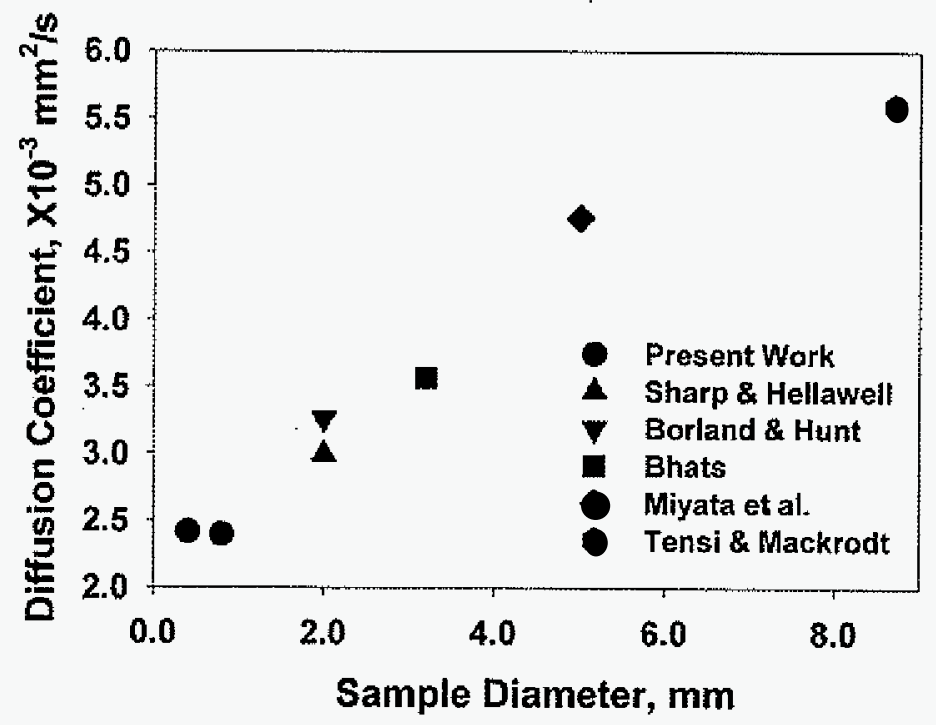

Fig. 6 A correlation between the effective diffusion coefficient and the diameter of the sample used in experiments of AI-Cu alloys [6]. 
model by Karma et. al. ${ }^{[17]}$, which include the convection effect. Through iterative calculation of the composition profiles, continual comparison with experimental measurements and global minimization of the difference between the measurement and calculation, the derived diffusion coefficient in the liquid is found to be $D_{l}=5.4 \times 10^{-9} \mathrm{~m}^{2} / \mathrm{s}$ and the boundary layer thickness is 4.0 for the $\phi 0.4 \mathrm{~mm}$ sample, 1.0 for the $\phi 0.8 \mathrm{~mm}$ sample and 0.3 for the $\phi 1.5 \mathrm{~mm}$ sample. This experimental study also uncovers the important difference for the solidification behavior in alloy systems which contain a heavier or a lighter solute. For the lighter solute containing alloys, it is impossible to carry out the growth process controlled by diffusion only even though a capillary sample is used. Nonetheless, for a heavier solute containing alloy, diffusive growth can be obtained as long as the sample is thin enough.

\section{Acknowledgments}

This work was made possible by support from the Office of Basic Energy Science, Division of Materials Science, U.S. Department of Energy, under Contract No. DE-AC02$07 \mathrm{CH} 11358$.

\section{References}

1. J. B. Edwards, E. E. Hucke and J. J. Martin, Int. Metall. Rev., 13 (1968) 1-12.

2. K. Niwa, M. Shimoji, S. Kado, Y. Watanabe, and T. Yokokawa, J. Metals, 9(1) (1957) 96-101.

3. T. Ejima, T. Uamamura, N. Uchida, Y. Matsuzaki, and M. Nikaido, J. J. Inst. Metals, 44(3) (1980) 316-323.

4. M. P. Watson and J. D. Hunt, Metall. Trans., 8A(11) (1977) 1793-1798. 
5. S. J. Teng and J. Choi, Metall. Mater. Trans., in print.

6. J.H.Lee, S. Liu, H. Miyahara, R. Trivedi, Metall. Mater. Trans., 35B(5) (2004) 909-917.

7. J. D. Verhoeven, E.D. Gibson, and R.I. Griffith, Metall. Trans., 6B(3) (1975) 475-480.

8. R. M. Jordan and J. D. Hunt, Metal. Trans.A, 2A(12) (1971) 3401-3410.

9. R.M. Sharp and A. Hellawell, J. Cryst. Growth, 5(3) (1969) 155-161.

10. D. Froschhammer, H.M. Tensi, H. Zoller, U. Feurer, Metall. Trans. B, 11B(1) (1980) $169-171$.

11. T. Sato and G. Ohira, J. Cryst. Growth, 40(1) (1977) 78-89.

12. R. Trivedi, S. Liu, P. Mazumder, and E. Simsek, Sci. Tech. of Advanced Mater., 2(1) (2001) 309-320.

13. S. Liu, P. Mazumder and R.Trivedi, J. Cryst. Growth, 240(3-4) (2002) 560-568.

14. S. Liu, J. Li, J, Lee, and R. Trivedi, Phil. Mag., 86(24) (2006), 3717-3738.

15. J. Hui, R. Tiwari, X. Wu, S.N. Tewari, and R. Trivedi, Metall. Mater.Trans. A, 33A(11) (2002) 3499-3510.

16. S. Liu and R. Trivedi, Metall. Mater. Trans., 37A(11) (2006) 3293-3304.

17. A. Karma et al., Metall. Mater. Trans., 29A (5) (1998) 1457-1470.

18. F. Dean, J. R. Kerr and A. Hellawell, J. Institute of Metals, 90 (1962) 234-237.

19. V. G. Smith, W.A.Tiller ans J. W. Rutter, Canadian J. Phys., 33 (1995) 723-745.

20. J.J.Favier, Acta Metall., 29(1) (1981) 205-214.

21. J.A. Burton, R.C. Prim and W. P. Slichter, J. Chem. Phys., 21 (1953) 1987-1991.

22. L.O.Wilson, J. Cryst. Growth, 44(2) (1978) p.247-250.

23. M. Zeif and W.R.Wilcox, Fractional Solidification, Marcel Dekker inc. NY., 1967, p.8586. 
24. J. Chen, S.N. Tewari, G. Magadi, and H.C. De Groh III, Metall. Mater. Trans., vol. 34A(12) (2003) 2985-2990.

25. Y. Miyata, J. J. Inst. Metals, 11 (1983) 1004-1009.

26. B. N. Bhat, J. Crystal Growth, 28 (1975) 68-76. 